Pontifícia Universidade Católica $_{\text {a }}$

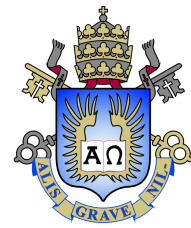

Thiago Augusto Lucas da Silva

A fórmula de Avila-Bochi-Herman e outros resultados relacionados

Dissertação apresentada como requisito parcial para a obtenção do grau de Mestre pelo Programa de Pós-graduação em Matemática da PUC-Rio .

Orientador: Prof. Silvius Klein 


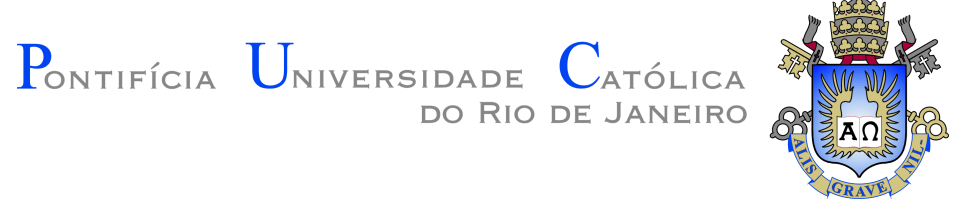

Thiago Augusto Lucas da Silva

\section{A fórmula de Avila-Bochi-Herman e outros resultados relacionados}

Dissertação apresentada como requisito parcial para a obtenção do grau de Mestre pelo Programa de Pós-graduação em Matemática da PUC-Rio . Aprovada pela Comissão Examinadora abaixo.

Prof. Silvius Klein

Orientador

Departamento de Matemática - PUC-Rio

Prof. Alexandre Tavares Baraviera

Departamento de Matemática - UFRGS

Prof. Marcos Craizer

Departamento de Matemática - PUC-Rio

Prof. Lorenzo Justiniano Díaz Casado

Departamento de Matemática - PUC-Rio

Prof. Rafael Oswaldo Ruggiero Rodriguez

Departamento de Matemática - PUC-Rio

Rio de Janeiro, 10 de Setembro de 2020 
Todos os direitos reservados. É proibida a reprodução total ou parcial do trabalho sem autorização da universidade, do autor e do orientador.

Thiago Augusto Lucas da Silva

Graduado em Bacharelado em Matemática pela Universidade Federal Fluminense, Volta Redonda-RJ, Brasil.

Ficha Catalográfica

Lucas, Thiago

A fórmula de Avila-Bochi-Herman e outros resultados relacionados / Thiago Augusto Lucas da Silva; orientador: Silvius Klein. - Rio de janeiro: PUC-Rio, Departamento de Matemática, 2020.

v., 100 f: il. color. ; $30 \mathrm{~cm}$

Dissertação (mestrado) - Pontifícia Universidade Católica do Rio de Janeiro, Departamento de Matemática.

Inclui bibliografia

1. Sistemas Dinâmicos;. 2. Teoria Ergódica;. 3. Cociclos Lineares;. 4. Expoentes de Lyapunov;. 5. Fórmula de AvilaBochi-Herman.. I. Klein, Silvius. II. Pontifícia Universidade Católica do Rio de Janeiro. Departamento de Matemática. III. Título. 
A Deus, a Bruna, minha família e amigos, por todo suporte ao longo desta jornada. 


\section{Agradecimentos}

Primeiramente, agradeço a Deus por me amparar e possibilitar ao longo desta jornada.

Agradeço as pessoas mais importantes em minha vida, minha família: Ana, Augusto, Bruna, Thaís, Daniele, Márcio e Maria Helena, pelo amor, carinho e cuidado, os quais me tornaram quem sou.

Gostaria de agradecer especialmente ao meu orientador, Silvius Klein, por todo apoio, suporte, paciência e conhecimento compartilhado, tornando possível a realização deste trabalho. Assim como todo o corpo docente, o qual contribuiu grandemente para o meu amadurecimento matemático.

Agradeço ainda aos membros da banca examinadora: Alexandre Baraviera, Marcos Craizer, Lorenzo Díaz e Rafael Ruggiero, pelo aceite ao convite e por toda contribuição para este projeto.

Meu sincero agradecimento também dedico a Creuza, assim como o corpo administrativo do Departamento de Matemática, pelo auxílio e boa vontade em lidar com toda a burocracia necessária.

Sou grato a todos os amigos da PUC-Rio, por todos os momentos compartilhados, acadêmicos ou não, os quais tornaram mais leve e prazerosa esta experiência. Em especial a Lucas, Hugo, Daniel, Thiago, Tahyz, Jéssica, Aimeé, Samuel, Igor e João.

Por fim, agradeço aos amigos que tornaram-se parte de minha família : Wagner e William, os quais sempre estiveram comigo e ainda se fazem presente.

O presente trabalho foi realizado com apoio da Coordenação de Aperfeiçoamento de Pessoal de Nível Superior - Brasil (CAPES) - Código de Financiamento 001. 


\section{Resumo}

Lucas, Thiago; Klein, Silvius. A fórmula de Avila-Bochi-Herman e outros resultados relacionados. Rio de Janeiro, 2020. 100p. Dissertação de Mestrado - Departamento de Matemática, Pontifícia Universidade Católica do Rio de Janeiro.

Os expoentes de Lyapunov são uma ferramenta bastante utilizada quando busca-se entender o comportamento de sistemas dinâmicos, em particular de cociclos lineares. De fato, concentramo-nos no expoente maximal, pois este determina o comportamento geral do sistema, de modo que sua positividade pode ser um indicativo de que estamos lidando com um sistema caótico. Nesse sentido estudamos um teorema provado por Michael Herman, que fornece uma cota inferior para o expoente de Lyapunov maximal de uma classe de cociclos lineares definidos por rotações no círculo. A prova deste resultado utiliza um processo de complexificação do cociclo e um argumento de subharmonicidade. Surpreendentemente, essa cota inferior é na verdade uma identidade, o que foi provado posteriormente por Avila e Bochi. Como será mostrado nesta dissertação, o argumento para obter a identidade depende crucialmente da harmonicidade, e não da mera subharmonicidade de certas funções associadas às iterações do cociclo.

\section{Palavras-chave}

Sistemas Dinâmicos; Teoria Ergódica; Cociclos Lineares; Expoentes de Lyapunov; Fórmula de Avila-Bochi-Herman. 


\section{Abstract}

Lucas, Thiago; Klein, Silvius (Advisor). Avila-Bochi-Herman's formula and other related results. Rio de Janeiro, 2020. 100p. Dissertação de Mestrado - Departamento de Matemática, Pontifícia Universidade Católica do Rio de Janeiro.

Lyapunov exponents are a widely used tool when trying to understand the behavior of dynamical systems in general, and in particular that of linear cocycles. We focus on the maximal exponent, as it determines the general behavior of the system, in that its positivity can be an indication that we are dealing with a chaotic system. In this sense, we study a theorem obtained by Michael Herman, providing a lower bound on the maximal Lyapunov exponent of a class of linear cocycles defined by circle rotations. The proof of this result employs the complexification of the cocycle and an argument based on subharmonicity. Surprisingly, this lower bound is in fact an identity, which was proven later by Avila and Bochi. As it will be shown in this dissertation, the argument for obtaining this identity depends crucially on the harmonicity, as opposed to the mere subharmonicity of certain functions associated with the iterates of the cocycle.

\section{Keywords}

Dynamical Systems; Ergodic Theory; Linear Cocycles; Lyapunov Exponents; Avila-Bochi-Herman's Formula. 


\section{Sumário}

1 Introdução 11

2 Subharmonicidade e a Desigualdade de Herman $\quad 15$

3 Recapitulação de conceitos $\quad 19$

$\begin{array}{lll}3.1 & \text { Sistemas ergódicos } & 19\end{array}$

3.2 Cociclos Lineares e os Expoentes de Lyapunov 23

3.3 Funções harmônicas e outros conceitos de Análise Complexa 26

3.4 O Grupo Fundamental e outros conceitos de Topologia Algébrica 31

4 A demonstração da Fórmula de Avila-Bochi-Herman 50

$\begin{array}{llr}5 & \text { Referências bibliográficas } & 99\end{array}$ 


\section{Lista de figuras}

Figura 4.1 Caminho $C_{r}=[-r, r] * \gamma_{r}$ 
Feliz é o homem que persevera na provação, porque depois de aprovado receberá a coroa da vida, que Deus prometeu aos que o amam.

Tiago 1:12, Bíblia Sagrada. 


\section{1 \\ Introdução}

Um sistema dinâmico pode ser definido informalmente como um conjunto de estados que evoluem com o tempo, de acordo com uma regra determinística, e a maneira como a variável tempo é modelada, sendo como um número real ou um número inteiro, determina de que modo o estado evolui, e ainda nos permite definir dois tipos de sistemas dinâmicos, os contínuos e os discretos, respectivamente. Neste trabalho estaremos exclusivamente interessados nos sistemas dinâmicos discretos, em que no intervalo de tempo entre dois instantes o estado permanece constante, os quais geralmente são dados por uma transformação $T$, definida sobre um espaço $X$ para o mesmo, de modo a ser interpretado como o mapa $T: X \rightarrow X$.

Um dos principais objetivos da teoria é justamente estudar o comportamento destas dinâmicas à medida que o tempo passa, e fazemos isto, de um modo geral, por meio da órbita de pontos em $X$, a qual é dada para um ponto $x \in X$, como sendo o conjunto formado pelos pontos $x, T(x), T^{2}(x):=T(T(x)), \ldots$ que representam também a trajetória do ponto $x$ no espaço $X$, sob a aplicação $T$, em que $T$ se tratando de uma aplicação invertível, faz sentido considerarmos os iterados passados. Prosseguindo com a teoria, ao "munirmos" um sistema dinâmico discreto $T: X \rightarrow X$ com uma medida de probabilidade $\mu$, denotamos esta tripla por $(X, \mu, T)$ e estamos interessados em exigir certas propriedades básicas deste sistema, como o fato de $T$ ser uma aplicação $\mu$-invariante, uma dinâmica que preserva medida, de modo que para todo subconjunto $A \subseteq X \mu$-mensurável, tenhamos $T^{-1}(A) \mu$-mensurável e tal que $\mu(A)=\mu\left(T^{-1}(A)\right)$.

Um dos principais exemplos deste tipo de dinâmica, que acabam por ser o principal objeto de estudo deste trabalho, são os cociclos lineares, em que para um sistema dinâmico que preserva medida $(X, \mu, T)$ e uma aplicação $\mu$-mensurável $A: X \rightarrow \mathrm{GL}(d, \mathbb{R})$, o cociclo linear definido por $A$ sobre $T$ é dado pela aplicação

$$
\begin{aligned}
F_{A}: X \times \mathbb{R}^{d} & \longrightarrow X \times \mathbb{R}^{d} \\
(x, v) & \longmapsto(T(x), A(x) v) .
\end{aligned}
$$


Seu $n$-ésimo iterado é definido por $F_{A}^{(n)}(x, v)=\left(T^{n}(x), A^{(n)}(x) v\right)$, sendo

$$
A^{(n)}(x):=A\left(T^{n-1}(x)\right) \ldots A(T(x)) A(x),
$$

em que é comum cometermos o abuso de nos referirmos ao cociclo apenas por $A$. A Teoria Ergódica, área na qual este trabalho se concentra, também se dedica ao estudo dos cociclos mencionados e busca estudar os sistemas dinâmicos por meio de propriedades estatísticas dos mesmos, no sentido de que estas propriedades são expressas através do comportamento de médias temporais ao longo das trajetórias desta dinâmica, sendo uma das principais ferramentas utilizadas, os expoentes de Lyapunov, e em particular o expoente de Lyapunov maximal.

Como pode-se imaginar, frequentemente lidamos com sistemas dinâmicos ergódicos $(X, \mu, T)$, os quais além da propriedade que descrevemos anteriormente, grosso modo, caracterizam-se por não serem decompostos, no sentido de que $(X, \mu)$ sendo um espaço de probabilidade, $T$ sendo $\mu$-invariante e para todo $A \subset X \mu$-mensurável tal que $T^{-1}(A)=A$, tenhamos $\mu(A)=0$ ou $\mu(A)=1$. Neste caso, sob determinada condição de integrabilidade, o expoente de Lyapunov maximal de um cociclo $A: X \rightarrow \mathrm{GL}(d, R)$ é definido como o limite

$$
L(A)=\lim _{n \rightarrow \infty} \frac{1}{n} \log \left\|A^{(n)}(x)\right\|,
$$

que existe e é constante para $\mu$-q.t.p. $x \in X$. Além disso,

$$
L(A)=\lim _{n \rightarrow \infty} \int_{X} \frac{1}{n} \log \left\|A^{(n)}(x)\right\| d \mu(x) .
$$

Caminhando neste sentido, no Capítulo 2 estudaremos um teorema provado por Michael Herman, o qual chamaremos de Desigualdade de Herman, que fornece uma cota inferior para o expoente de Lyapunov maximal de uma determinada classe de cociclos.

Teorema 1.1 (Desigualdade de Herman) Seja $\mathbb{T}=\mathbb{R} / \mathbb{Z}$ o toro unidimensional, $T: \mathbb{T} \rightarrow \mathbb{T}$ uma translação irracional e $A: \mathbb{T} \rightarrow S L(2, \mathbb{R})$ uma função da forma $A(x)=A_{0} R_{2 \pi x}$, em que $A_{0}=\left(\begin{array}{cc}\delta & 0 \\ 0 & \delta^{-1}\end{array}\right)$ para algum $\delta>0 e$ $R_{\theta}=\left(\begin{array}{cc}\cos \theta & -\sin \theta \\ \sin \theta & \cos \theta\end{array}\right) \cdot$ Então,

$$
L(A) \geq \log \left(\frac{\delta+\delta^{-1}}{2}\right)
$$


Perceba que de fato é interessante obtermos ao menos uma cota inferior para o expoente de Lyapunov maximal, pois sua positividade é geralmente tida como uma indicação de que o sistema é caótico. Como veremos, trabalharemos em grande parte do tempo sobre o espaço de matrizes $\mathrm{SL}(2, \mathbb{R})$, e para o mesmo definimos a seguinte quantidade

$$
N(A)=\log \left(\frac{\|A\|+\|A\|^{-1}}{2}\right)
$$

que pode ser interpretada como uma "taxa média exponencial de expansão" da matriz $A$. Com respeito a esta quantidade exploramos alguns resultados como o teorema a seguir, em que $\rho$ é tido como o raio espectral de uma matriz.

Teorema 1.2 Sejam $A_{1}, \ldots, A_{n} \in S L(2, \mathbb{R})$. Então

$$
\frac{1}{2 \pi} \int_{0}^{2 \pi} \log \rho\left(A_{n} R_{\theta} \cdots A_{1} R_{\theta}\right) d \theta=\sum_{j=1}^{n} N\left(A_{j}\right) .
$$

O resultado determinístico enunciado acima é particularmente relevante, uma vez que ele representa o ponto chave na demonstração da Fórmula de Avila-Bochi-Herman, teorema no qual os esforços deste trabalho foram concentrados, já que por meio deste conseguimos obter informações a respeito do expoente de Lyapunov maximal de determinados cociclos. Perceba que a partir de um dado cociclo $A: X \rightarrow \mathrm{SL}(2, \mathbb{R})$ podemos definir um novo cociclo linear

$$
\begin{aligned}
A R_{\theta}: X & \longrightarrow \mathrm{SL}(2, \mathbb{R}) \\
x & \longmapsto\left(A R_{\theta}\right)(x)=A(x) R_{\theta},
\end{aligned}
$$

e é justamente a estes cociclos que o teorema se refere.

Teorema 1.3 (A Fórmula de Avila-Bochi-Herman) Seja $(X, \mu)$ um espaço de probabilidade, $T: X \rightarrow X$ uma transformação ergódica e $A: X \rightarrow S L(2, \mathbb{R})$ um cociclo linear que satisfaz a condição de integrabilidade $\int_{X} \log \|A\| d \mu<\infty$. Então

$$
\frac{1}{2 \pi} \int_{0}^{2 \pi} L\left(A R_{\theta}\right) d \theta=\int_{X} \log \left(\frac{\|A(x)\|+\|A(x)\|^{-1}}{2}\right) d \mu(x) .
$$


Intuitivamente o Teorema 1.3 fornece uma fórmula, não exatamente para o expoente de Lyapunov maximal do cociclo dado, mas para a média dos expoentes de Lyapunov maximal das rotações do cociclo dado. Além disto, não é difícil ver que, no caso particular do cociclo considerado na Desigualdade de Herman, a fórmula de A-B-H mostra que esta desigualdade é, na verdade, uma igualdade. Vale ressaltar que para estudar este resultado, estamos lidando com a prova fornecida por Avila e Bochi, a qual pode ser encontrada em [2]. No entanto, este mesmo resultado foi provado por Baraviera, Dias e Duarte, com argumentos bastante distintos aos encontrados aqui, os quais, pode-se dizer, mais geométricos, como pode ser visto em [4].

A posteriori, esta fórmula foi aplicada em certos resultados, essencialmente com o objetivo de expressar a positividade do expoente de Lyapunov maximal, como pode ser visto em [18] e [1]. É interessante mencionar que existe uma generalização deste resultado, veja em [14], a qual se dá em dimensão superior a dois e para cociclos sob hipóteses mais gerais.

O restante do trabalho é dividido como se segue. No capítulo 2, que pode ser considerado uma prévia, abordaremos em detalhes a Desigualdade de Herman que mencionamos acima, dando foco ao argumento de subharmonicidade utilizado. No capítulo 3 faremos uma breve revisão dos conceitos relevantes para o desenvolvimento do trabalho. Por fim, o capítulo 4 é construído de modo a provarmos em detalhes a Fórmula de Avila-Bochi-Herman. 


\section{2}

\section{Subharmonicidade e a Desigualdade de Herman}

No presente capítulo abordaremos a Desigualdade de Herman, a qual fornece uma cota inferior para o expoente de Lyapunov maximal de uma classe de cociclos lineares, definidos com respeito a translações irracionais no toro unidimensional. A prova do teorema é dada por um processo de complexificação do cociclo em questão, de modo a obtermos uma função holomorfa, para então a partir desta, por meio da Fórmula de Jensen, obter uma função subharmônica e finalmente expressarmos a desigualdade desejada.

Existem maneiras equivalentes de caracterizarmos uma função subharmônica num domínio do plano complexo. Intuitivamente este conceito nos diz que, definida sob tais circunstâncias, uma função subharmônica avaliada em um ponto não é maior do que a média dos valores dessa função percorrendo um círculo centrado no referido ponto. Este conceito, geralmente, pode estar definido sobre hipóteses mais fracas do que as apresentadas abaixo, no entanto assumiremos hipóteses mais fortes, afim de definirmos subharmônicidade da maneira a qual nos será mais útil.

Definição 2.1 Seja $\Omega \subset \mathbb{C}$ um conjunto aberto e seja $u: \Omega \rightarrow[-\infty, \infty)$ uma função contínua. Dizemos que u é uma função subharmônica se para todo $z_{0} \in \Omega$ e $r>0$ tais que o disco fechado $\bar{D}\left(z_{0}, r\right) \subset \Omega$, tem-se

$$
u\left(z_{0}\right) \leq \frac{1}{2 \pi} \int_{0}^{2 \pi} u\left(z_{0}+r e^{i \theta}\right) d \theta
$$

A Fórmula de Jensen é um resultado de grande relevância neste capítulo, pois é o responsável por nos fornecer a função subharmônica, a qual implica a desigualdade obtida pelo teorema proposto por Herman. De fato, esta nos diz que uma função holomorfa $f$, definida sobre uma região qualquer do plano complexo, que contém um disco fechado de centro $z_{0}$ e raio $r$, satisfaz a seguinte propriedade

$$
\log \left|f\left(z_{0}\right)\right| \leq \frac{1}{2 \pi} \int_{0}^{2 \pi} \log \left|f\left(z_{0}+r e^{i \theta}\right)\right| d \theta .
$$

Portanto, dada uma função holomorfa $f$ em $\Omega \subset \mathbb{C}, \log |f|$ é subharmônica.

Um exemplo importante desta consequência se dá para funções da forma $A: \Omega \subset \mathbb{C} \rightarrow \operatorname{Mat}(d, \mathbb{R})$, definidas sobre um aberto $\Omega$ e ainda holomorfas, 
no sentido de que para todo $z \in \Omega$, todas as entradas da matriz $A(z)$ são funções holomorfas de $z \in \Omega$, já que neste cenário a função $f(z):=\log \|A(z)\|$ é subharmônica (veja a Proposição 3.30).

Teorema 2.2 (Desigualdade de Herman) Seja $\mathbb{T}=\mathbb{R} / \mathbb{Z}$ o toro unidimensional, $T: \mathbb{T} \rightarrow \mathbb{T}$ uma translação irracional e seja $A: \mathbb{T} \rightarrow S L(2, \mathbb{R})$ uma função da forma $A(x)=A_{0} R_{2 \pi x}$ em que $A_{0}=\left(\begin{array}{cc}\delta & 0 \\ 0 & \delta^{-1}\end{array}\right)$ para algum $\delta>0$ e $R_{\theta}=\left(\begin{array}{cc}\cos \theta & -\sin \theta \\ \sin \theta & \cos \theta\end{array}\right) \cdot$ Então,

$$
L(A) \geq \log \left(\frac{\delta+\delta^{-1}}{2}\right)
$$

em que $L(A)$ é o expoente de Lyapunov maximal do cociclo definido por $A$, relativo à unica medida de probabilidade $m$, invariante por $T$.

Prova. Seja $t \in \mathbb{R} \backslash \mathbb{Q}$ tal que $T$ é uma translação por $t$ e $\omega:=2 \pi t$. Então

$$
\begin{aligned}
L(A) & =\lim _{n \rightarrow \infty} \frac{1}{n} \int_{0}^{1} \log \left\|A^{(n)}(y)\right\| d m(y) \\
& =\lim _{n \rightarrow \infty} \frac{1}{2 \pi n} \int_{0}^{2 \pi} \log \left\|A^{(n)}\left(\frac{x}{2 \pi}\right)\right\| d x \\
& =\lim _{n \rightarrow \infty} \frac{1}{2 \pi n} \int_{0}^{2 \pi} \log \left\|A\left(T^{n-1}\left(\frac{x}{2 \pi}\right)\right) \cdots A\left(T\left(\frac{x}{2 \pi}\right)\right) A\left(\frac{x}{2 \pi}\right)\right\| d x \\
& =\lim _{n \rightarrow \infty} \frac{1}{2 \pi n} \int_{0}^{2 \pi} \log \left\|A_{0} R_{2 \pi T^{n-1}\left(\frac{x}{2 \pi}\right)} \cdots A_{0} R_{2 \pi T\left(\frac{x}{2 \pi}\right)} A_{0} R_{x}\right\| d x \\
& =\lim _{n \rightarrow \infty} \frac{1}{2 \pi n} \int_{0}^{2 \pi} \log \left\|A_{0} R_{x+(n-1) \omega} \cdots A_{0} R_{(x+\omega)} A_{0} R_{x}\right\| d x,
\end{aligned}
$$

já que para todo $n \in \mathbb{Z}$ temos $2 \pi T^{n}\left(\frac{x}{2 \pi}\right)=2 \pi\left(\frac{x}{2 \pi}+n t\right)=x+n 2 \pi t=x+n \omega$. Como dito, a ideia desta prova é estendermos a função da última integral, $\log \left\|A_{0} R_{x+(n-1) \omega} \cdots A_{0} R_{(x+\omega)} A_{0} R_{x}\right\|$, à uma função subharmônica no plano complexo e então deduzirmos a afirmação do teorema pela caracterização de funções subharmônicas, como descrito na Definição 2.1. Começaremos por complexificar a matriz de rotação $R_{\theta}$. Note que para $z=e^{i \theta}=\cos \theta+i \sin \theta$ temos,

$$
\frac{z+z^{-1}}{2}=\cos \theta, \quad \text { e } \quad \frac{z-z^{-1}}{2 i}=\sin \theta .
$$

Assim, defina a matriz

$$
S(z)=\left(\begin{array}{cc}
\frac{z+z^{-1}}{2} & -\frac{z-z^{-1}}{2 i} \\
\frac{z-z^{-1}}{2 i} & \frac{z+z^{-1}}{2}
\end{array}\right) \quad \text { para } z \in \mathbb{C}^{*}
$$


a qual possui uma singularidade, e defina ainda

$$
\mathscr{R}(z)=z S(z)=\left(\begin{array}{cc}
\frac{z^{2}+1}{2} & \frac{-z^{2}+1}{2 i} \\
\frac{z^{2}-1}{2 i} & \frac{z^{2}+1}{2}
\end{array}\right) \quad \text { para } z \in \mathbb{C}
$$

com um fator em relação à $S$, no caso $z$, porém sem singularidades, e perceba ainda que $\mathscr{R}\left(e^{i \theta}\right)=e^{i \theta} S\left(e^{i \theta}\right)=e^{i \theta} R_{\theta}$, para todo $\theta \in \mathbb{R}$. Portanto, faz sentido considerarmos $z \mapsto \mathscr{R}(z)$ como um tipo de extensão holomorfa da família de rotações $\theta \mapsto R_{\theta}$. Agora defina

$$
C_{n}(z)=A_{0} \mathscr{R}\left(e^{(n-1) \omega i} z\right) \cdots A_{0} \mathscr{R}\left(e^{\omega i} z\right) A_{0} \mathscr{R}(z)
$$

Então,

$$
\begin{aligned}
C_{n}\left(e^{i x}\right) & =A_{0} \mathscr{R}\left(e^{i(x+(n-1) \omega)}\right) \cdots A_{0} \mathscr{R}\left(e^{i(x+\omega)}\right) A_{0} \mathscr{R}\left(e^{i x}\right) \\
& =A_{0} e^{i(x+(n-1) \omega)} R_{x+(n-1) \omega} \cdots A_{0} e^{i(x+\omega)} R_{x+\omega} A_{0} e^{i x} R_{x} \\
& =\prod_{j=0}^{n-1} e^{i(x+j \omega)}\left(A_{0} R_{2 \pi T^{n-1}\left(\frac{x}{2 \pi}\right)} \cdots A_{0} R_{2 \pi T\left(\frac{x}{2 \pi}\right)} A_{0} R_{x}\right) \\
& =e^{i \sum_{j=0}^{n-1} i(x+j \omega)} A^{(n)}\left(\frac{x}{2 \pi}\right) \\
& =e^{i\left(n x+n(n-1) \frac{\omega}{2}\right)} A^{(n)}\left(\frac{x}{2 \pi}\right) .
\end{aligned}
$$

Assim, para $\tau=n x+n(n-1) \frac{\omega}{2}$ temos $C_{n}\left(e^{i x}\right)=e^{i \tau} A^{(n)}\left(\frac{x}{2 \pi}\right)$ e com isso $\left\|C_{n}\left(e^{i x}\right)\right\|=\left\|e^{i \tau} A^{(n)}\left(\frac{x}{2 \pi}\right)\right\|=\left|e^{i \tau}\right|\left\|A^{(n)}\left(\frac{x}{2 \pi}\right)\right\|=\left\|A^{(n)}\left(\frac{x}{2 \pi}\right)\right\|$. Portanto,

$$
L(A)=\lim _{n \rightarrow \infty} \frac{1}{2 \pi n} \int_{0}^{2 \pi} \log \left\|C_{n}\left(e^{i x}\right)\right\| d x .
$$

Note que a função $z \mapsto C_{n}(z)$ é holomorfa em toda parte, no sentido de que cada entrada da matriz é uma função holomorfa em toda parte, o que fica claro já que cada entrada será um polinômio. Então, como já mencionado, pela Proposição 3.30, para todo $n \in \mathbb{N}$ a função $z \mapsto \log \left\|C_{n}(z)\right\|$ é subharmônica. Desta maneira,

$$
\begin{aligned}
L(A)=\lim _{n \rightarrow \infty} \frac{1}{2 \pi n} \int_{0}^{2 \pi} \log \left\|C_{n}\left(e^{i x}\right)\right\| d x & \geq \lim _{n \rightarrow \infty} \frac{1}{n} \log \left\|C_{n}(0)\right\| \\
& =\lim _{n \rightarrow \infty} \frac{1}{n} \log \left\|\left(A_{0} \mathscr{R}(0)\right)^{n}\right\| .
\end{aligned}
$$

Já pela Proposição 4.22, que é uma consequência direta da fórmula de Gelfand temos

$$
\lim _{n \rightarrow \infty} \frac{1}{n} \log \left\|\left(A_{0} \mathscr{R}(0)\right)^{n}\right\|=\log \rho\left(A_{0} \mathscr{R}(0)\right),
$$


e como

$$
A_{0} \mathscr{R}(0)=\left(\begin{array}{cc}
\frac{\delta}{2} & -\frac{\delta i}{2} \\
\frac{\delta^{-1} i}{2} & \frac{\delta^{-1}}{2}
\end{array}\right)
$$

segue que o polinômio característico de $A_{0} \mathscr{R}(0)$ é $=\lambda^{2}-\lambda\left(\frac{\delta+\delta^{-1}}{2}\right)$. Deste modo, os autovalores de $A_{0} \mathscr{R}(0)$ são 0 e $\frac{\delta+\delta^{-1}}{2}$ e como $\delta>0$, tem-se $\rho\left(A_{0} \mathscr{R}(0)\right)=\frac{\delta+\delta^{-1}}{2}$. Portanto, por 2-1 e 2-2 obtemos

$$
L(A) \geq \log \left(\frac{\delta+\delta^{-1}}{2}\right)
$$

O fato interessante é que no decorrer deste trabalho, mais precisamente no capítulo 4, veremos que em um cenário até mais abrangente, no qual o Teorema de Herman provado acima ainda se encaixa, conseguimos que as funções definidas com respeito aos iterados dos cociclos lineares em questão, sejam estendidas à uma função na realidade harmônica, ao invés de apenas subharmônica, e deste modo conseguimos obter explicitamente o expoente de Lyapunov maximal de tais cociclos. 


\section{3}

\section{Recapitulação de conceitos}

Este capítulo tratará da revisão de conceitos indispensáveis ao entendimento do trabalho. A revisão será dividida em seções, as quais correspondem basicamente as áreas da matemática que daremos ênfase.

A Seção 3.1 busca familiarizar o leitor, de uma forma geral, aos conceitos introdutórios de sistemas dinâmicos ergódicos. Já a Seção 3.2, que de certa forma ainda está relacionada a seção anterior, tem o propósito de esclarecer a definição dos cociclos lineares, os quais são nosso principal objeto de trabalho, assim como o expoente de Lyapunov maximal destes cociclos, uma vez que o principal resultado desta dissertação diz respeito a obtermos este expoente explicitamente em um cenário particular. Na Seção 3.3 resgataremos alguns conceitos, assim como resultados relevantes, de Análise Complexa. Ainda nesta seção daremos foco ao conceito de harmônicidade de funções de uma variável complexa, pois a prova do principal teorema do trabalho, a Fórmula de AvilaBochi-Herman, depende essencialmente deste conceito. Por fim, na Seção 3.4 apresentaremos um pouco à respeito de Topologia Algébrica, sob o anseio de falarmos particularmente dos grupos fundamentais do toro unidimensional $\mathbb{T}$, do espaço projetivo $\operatorname{PSL}(2, \mathbb{R})$ e ainda de $\operatorname{PSL}(2, \mathbb{R}) \backslash\{[I]\}$.

\section{1}

\section{Sistemas ergódicos}

Como foi dito, tentaremos de forma breve introduzir os conceitos elementares à respeito dos sistemas dinâmicos ergódicos, vale ressaltar que todos conceitos e resultados apresentados podem ser vistos com mais detalhes em [16] e [17]. Começaremos por conceitos simples, que relacionam medidas à sistemas dinâmicos, para que possamos falar de certas propriedades, as quais exigimos dessa relação.

Definição 3.1 (Sistema Dinâmico que preserva medida) Considere uma transformação $T: X \rightarrow X$ mensurável, isto é, para todo conjunto mensurável $A \subset X, T^{-1}(A)$ também é mensurável, e seja $(X, \mu)$ um espaço de probabilidade. Dizemos que $(X, \mu, T)$ é um sistema dinâmico que preserva medida (ou apenas que $\mu$ é invariante por $T$, ou ainda que é $T$-invariante), se temos $\mu\left(T^{-1}(A)\right)=\mu(A)$, para qualquer conjunto mensurável $A \subset X$. 
Perceba, caso tivessemos para um sistema dinâmico que preserva medida $(X, \mu, T)$, um conjunto mensurável qualquer $A \subset X$ tal que $T^{-1}(A)=A$, então teríamos também $T^{-1}(X \backslash A)=X \backslash A$, assim poderíamos estudar a dinâmica $T$, estudando separadamente as dinâmicas mais simples $T: A \rightarrow A$ e $T: X \backslash A \rightarrow X \backslash A$. Contudo, essa maneira mais simples de tratar a dinâmica $T$, só faz sentido quando se tem $0<\mu(A)<1$, já que no caso em que $\mu(A)=0$ (ou equivalentemente $\mu(X \backslash A)=0$ ), faz sentido ignorarmos $A$ (ou equivalentemente $X \backslash A$ ), pois a Teoria da Medida nos permite neglicenciar conjuntos de medida zero. Isto nos traz então a ideia de estudarmos as dinâmicas que não podem ser decompostas como descrevemos acima, e a estas dinâmicas damos o nome de ergódica. Definimos então formalmente da seguinte maneira :

Definição 3.2 (Sistema Dinâmico Ergódico) Considere um sistema dinâmico $(X, \mu, T)$ que preserva medida. Dizemos que $(X, \mu, T)$ é um sistema dinâmico ergódico se, para qualquer conjunto mensurável $A \subset X$, tal que $T^{-1}(A)=A$, temos $\mu(A)=0$ ou $\mu(A)=1$.

Definição 3.3 (Função T-invariante) Considere o sistema dinâmico $(X, \mu, T)$ que preserva medida. Uma função $\mu$-mensurável $\varphi: X \rightarrow X$ é dita ser $T$ invariante, se $\varphi(T(x))=\varphi(x)$ para $\mu$-quase todo ponto $x \in X$.

A definição apresentada acima, nos permite ainda caracterizarmos os sistemas dinâmicos ergódicos da seguinte maneira. $(X, \mu, T)$ é um sistema dinâmico ergódico se qualquer função $\varphi: X \rightarrow \mathbb{R} \mu$-integrável, $T$-invariante é $\mu$-quase todo ponto $x \in X$ constante.

Observação 3.4 Em certos momentos, quando o espaço em questão $X$ e a medida tomada $\mu$ estiverem subentendidos, falaremos do sistema dinâmico ergódico apenas como a transformação $T$.

Agora que já temos conhecimento do que se trata um sistema dinâmico ergódico, introduziremos alguns exemplos conhecidos.

Exemplo 3.5 (Translação Irracional no Toro Unidimensional) Sabemos que o quociente $\mathbb{R} / \mathbb{Z}$ nos fornece um conjunto de classes $[x]$, com $x \in \mathbb{R}$, definido sobre a relação de equivalência $\sim$, a qual relaciona $x$ e y do seguinte modo, $x \sim y$ se, e somente se, $x-y \in \mathbb{Z}$. Também é sabido que podemos identificar $\mathbb{R} / \mathbb{Z}$ com o círculo unitário, isto é, o intervalo $[0,1]$ em que 0 e 1 estão identificados. Neste primeiro momento lidaremos com classes de equivalência, evitando tomar representantes, portanto assumiremos

$$
\mathbb{R} / \mathbb{Z}=\{[x] ; x \in \mathbb{R} \text { e } y \in \mathbb{R} \text { é tal que } y \in[x] \Leftrightarrow x-y \in \mathbb{Z}\}
$$


e podemos então definir a translação por $t \in \mathbb{R}$ da seguinte maneira

$$
\begin{aligned}
T: \mathbb{R} / \mathbb{Z} & \rightarrow \mathbb{R} / \mathbb{Z} \\
{[x] } & \mapsto[x+t]=[x]+[t]
\end{aligned}
$$

Considere ainda a projeção canônica, que associa a cada $x \in \mathbb{R}$ a sua classe de equivalência $[x] \in \mathbb{R} / \mathbb{Z}$ por

$$
\begin{aligned}
\pi: \mathbb{R} & \rightarrow \mathbb{R} / \mathbb{Z} \\
x & \mapsto[x]
\end{aligned}
$$

Definiremos então a medida de Lebesgue $\mu$ em $\mathbb{R} / \mathbb{Z}$, isto é, a medida de Lebesgue no círculo. Considere por $m$ a medida de Lebesgue na reta $\mathbb{R}$, dizemos que um conjunto $E \subset \mathbb{R} / \mathbb{Z}$ é $\mu$-mensurável se o conjunto $\pi^{-1}(E)$ é $m$-mensurável e definimos $\mu$ da seguinte maneira

$$
\mu(E)=m\left(\pi^{-1}(E) \cap[k, k+1)\right)
$$

para qualquer $k \in \mathbb{Z}$, ou seja, para um conjunto $E \subset \mathbb{R} / \mathbb{Z}$, tomamos a sua pré-imagem pela projeção $\pi$, a qual será a união infinita de intervalos na reta, e tomamos então um intervalo na reta, no caso o intervalo $[k, k+1)$ e portanto tomamos a medida de Lebesgue da interseção de $\pi^{-1}(E) \cap[k, k+1)$. Note que o lado esquerdo dessa igualdade não depende de $k$ e por definição temos

$$
\pi^{-1}(E) \cap[k, k+1)=\left(\pi^{-1}(E) \cap[0,1)\right)+k .
$$

Como a medida de Lebesgue na reta é invariante por translação, acabamos por ter

$$
\mu(E)=m\left(\pi^{-1}(E) \cap[0,1)\right)
$$

É nítido que $\mu$ é de probabilidade, pois $\mu(\mathbb{R} / \mathbb{Z})=m(\mathbb{R} \cap[0,1))=1$ e temos $(X, \mu, T)$ um sistema ergódico.

O segundo exemplo trata-se do Deslocamento de Bernoulli.

Exemplo 3.6 (Deslocamento de Bernoulli) Seja $(X, \mathcal{A}, \nu)$ um espaço de probabilidade qualquer. Considere o espaço $\Sigma=X^{\mathbb{N}}$, munido da $\sigma$-álgebra produto $\mathcal{B}=\mathcal{A}^{\mathbb{N}}$ e da medida produto $\mu=\nu^{\mathbb{N}}$. Então temos

$$
\Sigma=X^{\mathbb{N}}=\left\{\left(x_{k}\right)_{k \in \mathbb{N}}: x_{k} \in X \text { para todo } k\right\},
$$


e por definição a $\sigma$-álgebra $\mathcal{B}=\mathcal{A}^{\mathbb{N}}$ é gerada pelos cilindros

$$
\left[m ; A_{m}, \ldots, A_{n}\right]:=\left\{\left(x_{k}\right)_{k \in \mathbb{N}} \in \Sigma: x_{j} \in A_{j} \operatorname{para} j=m, \ldots, n\right\}
$$

sendo $m \leq n$ e $A_{j} \in \mathcal{A}$ para todo $j=m, \ldots, n$. Além disso, a medida $\mu$, definida sobre a $\sigma$-álgebra produto $\mathcal{B}$, a qual é gerada pela família $\mathcal{F}$ de todas as uniões finitas de cilindros disjuntos, que é uma álgebra, é caracterizada em $\Sigma$ por

$$
\mu\left(\left[m ; A_{m}, \ldots, A_{n}\right]\right)=\prod_{j=m}^{n} \nu\left(A_{j}\right) .
$$

Então temos o espaço de probabilidade $(\Sigma, \mathcal{B}, \mu)$, assim definimos o deslocamento de Bernoulli pelo sistema dinâmico $(\Sigma, \mu, \sigma)$, em que

$$
\begin{aligned}
& \sigma: \Sigma \longrightarrow \Sigma \\
&\left(x_{k}\right)_{k \in \mathbb{N}} \longmapsto\left(x_{k+1}\right)_{k \in \mathbb{N}},
\end{aligned}
$$

isto é, $\sigma\left(x_{0}, x_{1}, x_{2}, \ldots, x_{k}, \ldots\right)=\left(x_{1}, x_{2}, \ldots, x_{k}, \ldots\right)$, a imagem de uma sequência pelo deslocamento de Bernoulli é justamente essa sequência, deslocada uma unidade à esquerda. Note que

$$
\begin{aligned}
\sigma^{-1}\left(\left[m ; A_{m}, \ldots, A_{n}\right]\right) & =\left\{\left(x_{k}\right)_{k \in \mathbb{N}} \in \Sigma: \sigma\left(\left(x_{k}\right)_{k \in \mathbb{N}}\right) \in\left[m ; A_{m}, \ldots, A_{n}\right]\right\} \\
& =\left\{\left(x_{k}\right)_{k \in \mathbb{N}} \in \Sigma: x_{j+1} \in A_{j} \text { para } j=m, \ldots n\right\} \\
& =\left[m+1 ; A_{m}, \ldots, A_{n}\right]
\end{aligned}
$$

ou seja, a pré-imagem de qualquer cilindro pela aplicação $\sigma$ é um cilindro, e ainda

$$
\mu\left(\sigma^{-1}\left(\left[m ; A_{m}, \ldots, A_{n}\right]\right)\right)=\prod_{j=m}^{n} \nu\left(A_{j}\right)=\mu\left(\left[m+1 ; A_{m}, \ldots, A_{n}\right]\right)
$$

portanto $(\Sigma, \mu, \sigma)$ é um sistema dinâmico que preserva medida. Além disso, $(\Sigma, \mu, \sigma)$ é um sistema ergódico.

Observação 3.7 É importante ressaltar que os exemplos mais clássicos para o deslocamento de Bernoulli se dão quando consideramos $X$ um espaço finito de símbolos, como por exemplo $X=\{1, \ldots, d\}$, e damos um peso para cada elemento de $X$, como por exemplo $\nu(\{i\})=p_{i}>0$, de modo que $p_{1}+\cdots+p_{d}=1$.

Observação 3.8 Além disso, apresentamos o caso unilateral e de forma análoga tudo que foi mencionado acima continua a ser válido no cado bilareal, em que $\Sigma=X^{\mathbb{Z}}, \mathcal{B}=\mathcal{A}^{\mathbb{Z}}$ e $\mu=\nu^{\mathbb{Z}}$. 
Finalizaremos esta seção enunciando o Teorema Ergódico de Birkhoff, que é um resultado muito relevante em Teoria Ergódica, o qual trata de médias temporais e tem significativa relevância no caso em que a dinâmica é ergódica. Teorema 3.9 (Teorema de Birkhoff) Sejam $(X, \mu, T)$ um sistema ergódico e $\varphi: X \rightarrow \mathbb{R}$ uma função $\mu$-integrável. Então

$$
\lim _{n \rightarrow \infty} \frac{1}{n} \sum_{j=0}^{n-1} \varphi\left(T^{j}(x)\right)=\int_{X} \varphi(x) d \mu(x)
$$

para $\mu$-quase todo ponto $x \in X$.

\section{2}

\section{Cociclos Lineares e os Expoentes de Lyapunov}

Nesta seção traremos conceitos que em grande parte estão inseridos na Teoria Ergódica e assim ainda tem relação com sistemas dinâmicos ergódicos, porém merecem um destaque mais substancial. O conteúdo apresentado tem por base [15] e também [8].

Iniciaremos esta seção definindo o principal objeto de trabalho desta dissertação, os cociclos lineares. Seja $(X, \mu, T)$ um sistema dinâmico ergódico e $A: X \rightarrow \mathrm{GL}(d, \mathbb{R})$ uma função $\mu$-mensurável, em que $\mathrm{GL}(d, \mathbb{R})$ é o espaço das matrizes invertíveis $d \times d$ com coeficientes reais. Então o cociclo linear definido por $A$ sobre $T$, o qual denotamos por $F_{A}$, é definido como o produto torcido:

$$
\begin{aligned}
F_{A}: X \times \mathbb{R}^{d} & \rightarrow X \times \mathbb{R}^{d} \\
(x, v) & \mapsto(T(x), A(x) v)
\end{aligned}
$$

e então consequentemente, para $n \in \mathbb{N}$ obtemos o $n$-ésimo iterado de $F_{A}$ por $F_{A}^{(n)}(x, v)=\left(T^{n}(x), A\left(T^{n-1}(x)\right) \ldots A(T(x)) A(x) v\right)$. Denotaremos então

$$
F_{A}^{(n)}(x, v)=\left(T^{n}(x), A^{(n)}(x) v\right)
$$

em que

$$
A^{(n)}(x):=A\left(T^{n-1}(x)\right) \ldots A(T(x)) A(x) .
$$

Perceba que quando $T$ é inversível, de modo análogo, podemos definir seus iterados para trás.

Observação 3.10 É importante ressaltar que comumente cometemos o abuso de nos referirmos ao cociclo linear em questão apenas por A, ao invés da transformação $F_{A}$. 
Observação 3.11 Talvez seja relevante mencionarmos, para o estudo que se segue estaremos interessados no caso em que $d=2$ e também nos cociclos $A: X \rightarrow S L(2, \mathbb{R})$, em que $S L(2, R)$ é o espaço de matrizes $2 \times 2$ com coeficientes reais e determinante 1 .

A seguir daremos dois exemplos de cociclos lineares, os quais são relevantes para a teoria, de modo geral. O primeiro exemplo trata-se da classe de cociclos trabalhados na Desigualdade de Herman, Teorema 2.2, apresentado no Capítulo 2.

Exemplo 3.12 Seja $(\mathbb{T}, \mu, T)$ o sistema dinâmico ergódico em que $\mathbb{T}$ é o toro unidimensional, $\mu$ é a medida de Lebesgue no toro e $T$ uma translação irracional em $\mathbb{T}$, por $t$, isto é $T(x)=x+t \bmod 1$. Considere $A_{0}=\left(\begin{array}{cc}\delta & 0 \\ 0 & \delta^{-1}\end{array}\right)$, para algum $\delta>0$ e $R_{\theta}=\left(\begin{array}{cc}\cos \theta & -\sin \theta \\ \sin \theta & \cos \theta\end{array}\right)$ a matriz de rotação por um ângulo $\theta \in[0,2 \pi)$. Definimos entâa o cociclo linear $A: \mathbb{T} \rightarrow S L(2, \mathbb{R})$ definido por

$$
A(x)=A_{0} R_{2 \pi x}=\left(\begin{array}{cc}
\delta \cos (2 \pi x) & -\delta \sin (2 \pi x) \\
\delta^{-1} \sin (2 \pi x) & \delta^{-1} \cos (2 \pi x)
\end{array}\right)
$$

O outro exemplo a ser apresentado são os cociclos lineares aleatórios, os quais não serão trabalhados aqui, porém tem grande relevância no estudo da teoria.

Exemplo 3.13 (Cociclos lineares aleatórios) Considere um espaço de probabilidade $(X, \mathcal{A}, \nu)$, então como foi apresentado na seção anterior, Seção 3.1, obtemos o espaço de probabilidade $(\Sigma, \mathcal{B}, \mu)$ em que $\Sigma=X^{\mathbb{N}}, \mathcal{B}=\mathcal{A}^{\mathbb{N}}$ e $\mu=\nu^{\mathbb{N}}$. Assim, sendo $(\Sigma, \mu, \sigma)$ o deslocamento de Bernoulli como vimos no Exemplo 3.6, considere a função mensurável $\tilde{A}: X \rightarrow G L(d, \mathbb{R})$ e portanto definimos o cociclo linear aleatório pela função mensurável

$$
\begin{gathered}
A: \Sigma \\
\left(x_{k}\right)_{k \in \mathbb{N}} \longmapsto G L(d, \mathbb{R}) \\
\longrightarrow \tilde{A}\left(x_{0}\right)
\end{gathered}
$$

Deste modo, para todo $n \in \mathbb{N}$ temos

$$
\begin{aligned}
A^{(n)}\left(\left(x_{k}\right)_{k \in \mathbb{N}}\right) & =A\left(\sigma^{n-1}\left(\left(x_{k}\right)_{k \in \mathbb{N}}\right)\right) \ldots A\left(\sigma\left(\left(x_{k}\right)_{k \in \mathbb{N}}\right)\right) A\left(\left(x_{k}\right)_{k \in \mathbb{N}}\right) \\
& =A\left(\left(x_{k+(n-1)}\right)_{k \in \mathbb{N}}\right) \ldots A\left(\left(x_{k+1}\right)_{k \in \mathbb{N}}\right) A\left(\left(x_{k}\right)_{k \in \mathbb{N}}\right) \\
& =\tilde{A}\left(x_{n-1}\right) \ldots \tilde{A}\left(x_{1}\right) \tilde{A}\left(x_{0}\right) .
\end{aligned}
$$


Apresentado o conceito de cociclo linear, nosso objetivo para esta seção é definirmos o expoente de Lyapunov maximal para estes cociclos. Contudo, para que possamos, de fato, defini-los, precisaremos introduzir mais alguns conceitos, assim como outros resultados que nos permitam assumir sua existência.

Para todo o cenário que se segue considere $(X, \mu, T)$ um sistema dinâmico que preserva medida. A seguir definiremos o conceito de sequências subaditivas.

Definição 3.14 (Sequência Subaditiva) Dizemos que uma sequência $\left(\varphi_{n}\right)_{n \in \mathbb{N}}$ de funções $\mu-$ mensuráveis $\varphi_{n}: X \rightarrow \mathbb{R}$ é subaditiva, relativa à $T$, se para todo $m, n \in \mathbb{N}$ temos

$$
\varphi_{m+n} \leq \varphi_{n}+\varphi_{m} \circ T^{n}
$$

Lembre-se que considerando por $\|\cdot\|$ a norma do operador em $\operatorname{GL}(d, \mathbb{R})$, temos para quaisquer $A_{1}, A_{2} \in \mathrm{GL}(d, \mathbb{R})$ que $\left\|A_{1} A_{2}\right\| \leq\left\|A_{1}\right\|\left\|A_{2}\right\|$. Com isso, a seguir apresentaremos um exemplo relevante de uma sequência subaditiva de funções.

Exemplo 3.15 Considere o cociclo linear $A: X \rightarrow G L(d, \mathbb{R})$ e a sequência $\left(\varphi_{n}\right)_{n \in \mathbb{N}}$ em que

$$
\varphi_{n}=\log \left\|A^{(n)}\right\|
$$

para todo $n \in \mathbb{N}$ e $A^{(n)}(x)=A\left(T^{n-1}(x)\right) \ldots A(T(x)) A(x)$ para todo $x \in X$, como já havíamos definido. Então a sequência $\left(\varphi_{n}\right)_{n \in \mathbb{N}}$ é subaditiva. De fato, para todo $x \in X$

$$
\begin{aligned}
\varphi_{m+n}(x) & =\log \left\|A^{(m+n)}(x)\right\|=\log \left\|A\left(T^{m+n-1}\right)(x) \ldots A(T(x)) A(x)\right\| \\
& =\log \left\|A\left(T^{m-1}\left(T^{n}(x)\right)\right) \ldots A\left(T\left(T^{n}(x)\right)\right) A\left(T^{n}(x)\right) \ldots A(T(x)) A(x)\right\| \\
& =\log \left\|A^{(m)}\left(T^{n}(x)\right) A^{(n)}(x)\right\| \leq \log \left(\left\|A^{(m)}\left(T^{n}(x)\right)\right\|\left\|A^{(n)}(x)\right\|\right) \\
& =\log \left\|A^{(m)}\left(T^{n}(x)\right)\right\|+\log \left\|A^{(n)}(x)\right\|=\varphi_{m}\left(T^{n}(x)\right)+\varphi_{n}(x) \\
& =\varphi_{n}(x)+\left(\varphi_{m} \circ T^{n}\right)(x)
\end{aligned}
$$

O próximo resultado é um Teorema bastante conhecido em Teoria Ergódica, que acaba por ser o responsável em nos garantir a existência do expoente de Lyapunov maximal sob dadas circunstâncias.

Teorema 3.16 (Teorema de Kingman)

Sejam $(X, \mu, T)$ um sistema ergódico e $\left(\varphi_{n}\right)_{n \in \mathbb{N}}$ uma sequência subaditiva em 
$X$ tal que $\varphi_{1}^{+}$é $\mu$-integrável. Então para $\mu$-q.t.p. $x \in X$ temos

$$
\lim _{n \rightarrow \infty} \frac{1}{n} \varphi_{n}=\lim _{n \rightarrow \infty} \frac{1}{n} \int_{X} \varphi_{n} d \mu=\inf _{n \in \mathbb{N}} \frac{1}{n} \int_{X} \varphi_{n} d \mu
$$

Perceba que para um cociclo linear $A: X \rightarrow \mathrm{GL}(d, \mathbb{R})$, em que $A$ é uma função $\mu$-mensurável, temos $\varphi_{n}(x)=\log \left\|A^{(n)}(x)\right\|$ uma função $\mu$-mensurável para todo $n \in \mathbb{N}$, além disso, como vimos no Exemplo 3.15 esta sequência é subaditiva. Assim, se $\varphi_{1}^{+}=\log ^{+}\|A\|$ é $\mu$-integrável, então o Teorema de Kingman é aplicável e nos permite definir o conceito de expoente de Lyapunov maximal.

Definição 3.17 (Expoente de Lyapunov maximal) Sejam $(X, \mu, T)$ um sistema ergódico e $A: X \rightarrow G L(d, \mathbb{R})$ um cociclo linear tal que $\log ^{+}\|A\|$ é uma função $\mu$-integrável. Então o limite em $\mu$-q.t.p. $x \in X$

$$
L(A):=\lim _{n \rightarrow \infty} \frac{1}{n} \log \left\|A^{(n)}(x)\right\|=\lim _{n \rightarrow \infty} \int_{X} \frac{1}{n} \log \left\|A^{(n)}(x)\right\| d \mu(x)
$$

é chamado o expoente de Lyapunov maximal do cociclo A.

\section{3}

\section{Funções harmônicas e outros conceitos de Análise Complexa}

Com o intuito de falarmos a respeito de Análise Complexa, assim como temas relacionados, será feito um sucinto levantamento de resultados essenciais, os quais podem ser vistos também, e em detalhes, em [6] e [7]. Começaremos relembrando um conceito simples que é a definição de integral de linha, já que os demais resultados a usam com demasiada frequência.

Definição 3.18 (Integral de Linha) Seja $\Omega \subset \mathbb{C}$ um aberto. Dadas $\gamma:[a, b] \rightarrow \Omega$ e $f: \Omega \rightarrow \mathbb{C}$, em que $f$ é continua e $\gamma$ um caminho suave por partes. Definimos a integral de linha de $f$ ao longo do caminho $\gamma$ por

$$
\int_{\gamma} f(z) d z=\int_{a}^{b} f(\gamma(t)) \gamma^{\prime}(t) d t
$$

com a mudança $z=\gamma(t), d z=\gamma^{\prime}(t) d t$.

A integral de linha nos fornece uma proposição importante, que costuma ser bastante útil quando queremos complexificar integrais reais, afim de usar teoremas como o dos Resíduos, que será enunciado à frente. 
Proposição 3.19 Considere por l o comprimento de arco. Seja $\gamma$ e $f$ como descritas em 3.18. Então

$$
\left|\int_{\gamma} f(z) d z\right| \leq \sup _{z \in \gamma}|f(z)| l(\gamma)
$$

Agora, podemos então definir o conceito de índice.

Definição 3.20 (Índice) Seja $\gamma:[a, b] \rightarrow \mathbb{C}$ um caminho fechado e suave por partes. Então para todo $z \in \mathbb{C} \backslash \gamma$, definimos o índice da curva $\gamma$ em torno do ponto z por

$$
\operatorname{ind}_{\gamma}(z):=\frac{1}{2 \pi i} \int_{\gamma} \frac{1}{\xi-z} d \xi
$$

Observação 3.21 Vale ressaltar que o índice de uma curva $\gamma$ em torno de um ponto $z$, pode também ser interpretado como o número de voltas que a curva $\gamma$ dá em torno deste ponto, sendo positivo ou negativo a depender da orientação considerada, isto é, representa a variação do argumento ao longo de $\gamma$, no sentido de que parametrizando a curva $\gamma$ por coordenadas polares, de modo a obtermos em partícular para $z=0, \gamma(t)=r(t) e^{i \theta(t)}$ para $\theta \in[a, b] \in \mathbb{R}$ tem-se

$$
\operatorname{ind}_{\gamma}(0)=\frac{\theta(b)-\theta(a)}{2 \pi} .
$$

Para tratar dos próximos teoremas precisamos do conceito de conjunto estrelado.

Definição 3.22 (Conjunto Estrelado) Um conjunto $\Omega \subset \mathbb{C}$ é chamado estrelado, ou de forma estrela, se existe uma "estrela" $a \in \Omega$, tal que $[a, z] \subset \Omega$ para todo $z \in \Omega$, em que $[a, z]$ é o segmento de reta com extremos a e z.

Enunciaremos a seguir o teorema da fórmula de Cauchy local, a qual nos fornecerá um corolário relevante.

Teorema 3.23 (A Fórmula de Cauchy local) Sejam $\Omega \subset \mathbb{C}$ um domínio estrelado, $f: \Omega \rightarrow \mathbb{C}$ uma função holomorfa e $\gamma \subset \Omega$ um caminho fechado. Então para todo $z \in \Omega \backslash \gamma$ temos

$$
f(z) i n d_{\gamma}(z)=\frac{1}{2 \pi i} \int_{\gamma} \frac{f(\xi)}{\xi-z} d \xi .
$$


Corolário 3.24 (Propriedade do Valor Médio) Seja $\Omega \subset \mathbb{C}$ um aberto, $f$ uma função holomorfa em $\Omega$ e $\bar{D}\left(z_{0}, r\right) \subset \Omega$. Então,

$$
f\left(z_{0}\right)=\frac{1}{2 \pi} \int_{0}^{2 \pi} f\left(z_{0}+r e^{i \theta}\right) d \theta .
$$

Ao ponto da seção em que estamos, já faz sentido discorrermos a respeito das funções harmônicas de uma variável complexa. Considere a função $u: \Omega \subset \mathbb{R}^{2} \rightarrow \mathbb{R}$, em que $\Omega$ é um aberto. Dizemos então que $u(x, y)$ é uma função harmônica se todas as suas derivadas parciais de primeira e segunda ordem existem e são contínuas, e ainda, $u$ satisfaz a equação de Laplace, ou seja,

$$
\Delta u=\frac{\partial^{2} u}{\partial x^{2}}+\frac{\partial^{2} u}{\partial y^{2}}=0
$$

Observação 3.25 Lembremos que para qualquer função holomorfa $f=u+i v$, sua parte real, u, é uma função harmônica. Além disso, se $\Omega \subset \mathbb{C}$ for estrelado a recíproca também seria válida e teríamos que toda função harmônica é a parte real de uma função holomorfa.

À vista disso, como consequência direta da propriedade do Valor Médio, Corolário 3.24, tomando a parte real de ambos os lados, obtemos a seguinte caracterização à respeito das funções harmônicas :

Corolário 3.26 (Propriedade do Valor Médio para Funções Harmônicas) Sejam $\Omega \subset \mathbb{C}$ um aberto, estrelado e $u: \Omega \rightarrow \mathbb{C}$ harmônica. Se $\bar{D}\left(z_{0}, r\right) \subset \Omega$, então

$$
u\left(z_{0}\right)=\frac{1}{2 \pi} \int_{0}^{2 \pi} u\left(z_{0}+r e^{i \theta}\right) d \theta .
$$

Levando em conta que a subharmônicidade é um tema de relevância nesta área, voltaremos a falar brevemente deste tema, assim como resultados relacionados à funções subharmônicas, os quais foram brevemente descritos no Capítulo 2, porém agora em detalhes.

Definição 3.27 (Funções Subharmônicas) Sejam $\Omega \subset \mathbb{C}$ um conjunto aberto e $u: \Omega \rightarrow[-\infty,+\infty)$ uma função contínua. Dizemos que u é uma função subharmônica se, para todo $z_{0} \in \Omega$ e $r>0$ tais que $\bar{D}\left(z_{0}, r\right) \subset \Omega$, tem-se

$$
u\left(z_{0}\right) \leq \frac{1}{2 \pi} \int_{0}^{2 \pi} u\left(z_{0}+r e^{i \theta}\right) d \theta
$$


O próximo teorema a ser enunciado é a Fórmula de Jensen, este será responsável por nos fornecer um importante resultado à respeito de funções subharmônicas.

Teorema 3.28 (Fórmula de Jensen) Dada uma função $f$ holomorfa, definida em uma região do plano complexo a qual contém um disco fechado de centro $z_{0}$ e raio $r, \bar{D}\left(z_{0}, r\right)$, em que $a_{1}, \ldots, a_{n}$ são os zeros de $f$ dentro de $\bar{D}\left(z_{0}, r\right)$, repetidos de acordo com a multiplicidade e $f\left(z_{0}\right) \neq 0$, temos

$$
\log \left|f\left(z_{0}\right)\right| \leq \frac{1}{2 \pi} \int_{0}^{2 \pi} \log \left|f\left(z_{0}+r e^{i \theta}\right)\right| d \theta
$$

Observação 3.29 Note, pelo teorema da Fórmula de Jensen enunciado acima, podemos afirmar que para uma função holomorfa $f$, definida sobre um dominio $\Omega \subset \mathbb{C}$ aberto e sob tais circunstâncias, a função $\log |f|$ é subharmônica.

Esta observação torna-se ainda mais interessante quando se tratam de funções matriciais, sendo assim, é conveniente voltarmos a falar de uma proposição importante utilizada no Capítulo 2, em que naquela ocasião foi apenas discorrida em forma de texto, porém agora a enunciando e provando formalmente, esta pode ainda ser encontrada em [9].

Proposição 3.30 Seja $\Omega \subset \mathbb{C}$ um conjunto aberto e seja $A: \Omega \rightarrow \operatorname{Mat}(d, \mathbb{C})$ uma função holomorfa, no sentido de que cada entrada da matriz é uma função holomorfa de $z \in \Omega$. Então $f(z):=\log \|A(z)\|$ é subharmônica.

Prova. Primeiramente note que $f$ é uma função contínua, já que é a composição das funções $\log ,\|\cdot\|$ e $A$, sendo cada uma destas funções contínuas, e é claro que estamos tomando $A$ de modo que $A(z) \neq 0$, para todo $z \in \Omega$. Além disso, para cada $v \in \mathbb{R}^{d} \operatorname{com}|v|=1$, a função $z \mapsto\langle A(z) v, A(z) v\rangle$ é holomorfa, pois é uma combinação linear das entradas de $A(z)$, as quais são todas holomorfas. Portanto, para cada $v \in \mathbb{R}^{d} \operatorname{com}|v|=1$, a função $f_{v}: \Omega \rightarrow \mathbb{R}$ definida por

$$
f_{v}(z):=\log |A(z) v|
$$

é uma função subharmônica, por consequência direta da Fórmula de Jensen, Teorema 3.28. Mais ainda, note que dados $z_{0} \in \Omega$ e $r>0$ tais que $\bar{D}\left(z_{0}, r\right) \subset \Omega$, temos $f\left(z_{0}\right)=\log \left|A\left(z_{0}\right) v\right|$ e por definição, para todo $v$ como mencionado acima, a desigualdade

$$
f_{v}\left(z_{0}\right) \leq \frac{1}{2 \pi} \int_{0}^{2 \pi} f_{v}\left(z_{0}+r e^{i \theta}\right) d \theta
$$


Perceba que $f(z)=\log \|A(z)\|=\sup _{|v|=1} f_{v}(z)$ e ainda

$$
\begin{aligned}
f\left(z_{0}\right) & =\sup _{|v|=1} f_{v}(z) \leq \sup _{|v|=1} \frac{1}{2 \pi} \int_{0}^{2 \pi} f_{v}\left(z_{0}+r e^{i \theta}\right) d \theta \\
& \leq \frac{1}{2 \pi} \int_{0}^{2 \pi} \sup _{|v|=1} f_{v}\left(z_{0}+r e^{i \theta}\right) d \theta=\frac{1}{2 \pi} \int_{0}^{2 \pi} f\left(z_{0}+r e^{i \theta}\right) d \theta .
\end{aligned}
$$

Portanto $f(z)=\log \|A(z)\|$ é uma função subharmônica.

Continuando com a revisão enunciaremos um resultado bastante conhecido, que acaba por ser uma eficiente ferramenta no cálculo de integrais, o Teorema dos Resíduos, para tal relembraremos brevemente a definição de resíduo.

Definição 3.31 (Resíduo) Seja $f$ uma função holomorfa no disco perfurado $D^{*}\left(z_{0}, R\right)=D\left(z_{0}, R\right) \backslash\left\{z_{0}\right\}$, em que $\sum_{n=-\infty}^{\infty} c_{n}\left(z-z_{0}\right)^{n}$ é a sua expansão em série de Laurent. Então definimos o resíduo de $f$ no ponto $z_{0}$ por

$$
\operatorname{Res}\left(f, z_{0}\right):=c_{-1}=\frac{1}{2 \pi i} \int_{\left|z-z_{0}\right|=r} f(\xi) d \xi
$$

para todo $r \in(0, R)$.

Observação 3.32 Quando $z_{0}$ é um polo de $f$, de ordem 1, temos

$$
\operatorname{Res}\left(f, z_{0}\right)=\lim _{z \rightarrow z_{0}}\left(z-z_{0}\right) f(z)
$$

Teorema 3.33 (Teorema dos Resíduos) Sejam $\Omega \subset \mathbb{C}$ um aberto estrelado, $S=\left\{z_{1}, \ldots, z_{n}\right\} \subset \Omega$ um conjunto de pontos distintos tais que $f$ é holomorfa em $\Omega \backslash S$, e $\gamma \subset \Omega \backslash S$ um caminho fechado. Então,

$$
\int_{\gamma} f(z) d z=(2 \pi i) \sum_{k=1}^{n} \operatorname{Res}\left(f, z_{k}\right) i n d_{\gamma}\left(z_{k}\right) .
$$

O próximo resultado a ser apresentado é o Teorema de Rouché, que trata da quantidade de zeros de funções holomorfas dentro de uma dada região, as quais satisfazem certa condição no bordo desta região.

Observação 3.34 Sendo $f: \Omega \subset \mathbb{C} \rightarrow \mathbb{C}$, considere por

$$
Z(f)=\{z \in \Omega: f(z)=0\}
$$

e $Z_{f}$ o número total de zeros de $f$ em $\Omega$, contados com multiplicidade. 
Teorema 3.35 (Teorema de Rouché) Seja $\Omega \subset \mathbb{C}$ um aberto estrelado, $f, g$ funções holomorfas em $\Omega, S=Z(f) \cup Z(g)$ e $\gamma \subset \Omega \backslash S$ tal que ind $d_{\gamma}(a)=1$ para todo $a \in S$. Se $|f(z)-g(z)|<|f(z)|$ para todo $z \in \gamma$ então $Z_{f}=Z_{g}$.

O último resultado que exibiremos nesta seção nos permite afirmar que, para uma função definida sobre determinadas circunstâncias, o logaritmo desta possuirá um ramo holomorfo em dada região.

Teorema 3.36 Seja $\Omega \subset \mathbb{C}$ simplesmente conexo e $f$ holomorfa em $\Omega$ tal que $f(z) \neq 0$ para todo $z \in \Omega$. Então Log $f$ possui um ramo holomorfo em $\Omega$.

Observação 3.37 Uma consequência imediata do Teorema enunciado acima é o fato de $\sqrt{f}=\exp \left(\frac{1}{2} \log f\right)$ possuir um ramo holomorfo em $\Omega$.

\section{4}

\section{O Grupo Fundamental e outros conceitos de Topologia Algébrica}

A última seção deste capítulo destina-se a recordar conceitos de Topologia Algébrica, obviamente com ênfase especial no grupo fundamental, uma vez que o principal teorema deste trabalho faz uso de tais conceitos, porém também tratando de alguns resultados relacionados. Esta síntese pode ser esclarecida, em geral, em [13]e [11], juntamente com [10].

Começaremos definindo uma operação para caminhos, a qual chamaremos de concatenação. Para o cenário que se segue, considere $X$ e $Y$ como espaços topológicos, e sem perda de generalidade estaremos considerando caminhos sempre definidos sobre $[0,1]$.

Definição 3.38 (Caminho Reverso) Sendo $\gamma:[0,1] \rightarrow X$ um caminho em $X$, considere por $\bar{\gamma}:[0,1] \rightarrow X$ o caminho reverso de $\gamma$ em $X$, que consiste exatamente na mesma curva $\gamma$, porém parametrizada de modo a ter orientação contrária, isto é, $\bar{\gamma}(0)=\gamma(1)$ e $\bar{\gamma}(1)=\gamma(0)$.

Definição 3.39 (Concatenação de Caminhos) Sejam $\gamma, \beta:[0,1] \rightarrow X$ caminhos em $X$ tais que $\gamma(0)=x_{0}, \gamma(1)=\beta(0)=x_{1}$ e $\beta(1)=x_{2}$. Definimos a concatenação $\gamma * \beta$, de $\gamma$ e $\beta$, como sendo o caminho

$$
(\gamma * \beta)(t)= \begin{cases}\gamma(2 t), & t \in\left[0, \frac{1}{2}\right] \\ \beta(2 t-1), & t \in\left[\frac{1}{2}, 1\right]\end{cases}
$$

em que $(\gamma * \beta)(0)=\gamma(0)=x_{0} e(\gamma * \beta)(1)=\beta(1)=x_{2}$. 
A seguir definiremos o conceito de homotopia, primeiramente de uma forma geral para funções contínuas, e em seguida o caso partícular, o qual na verdade estaremos mais interessados, que é o conceito de homotopia de caminhos. De um modo geral, a homotopia está intuitivamente relacionada a possibilidade de deformarmos continuamente uma função contínua, em partícular um caminho, em outra dentro do espaço topológico em questão.

Definição 3.40 (Homotopia entre funções contínuas) Sejam $f, g: X \rightarrow Y$ funções contínuas. Dizemos que $f$ e $g$ são funções homotópicas, se existe uma função contínua $\Gamma: X \times[0,1] \rightarrow Y$ tal que $\Gamma(x, 0)=f(x)$ e $\Gamma(x, 1)=g(x)$, para todo $x \in X$. Chamamos $\Gamma$ de uma homotopia entre $f$ e $g$ e denotamos $f \simeq g$.

A homotopia entre funções contínuas nos fornece uma importante relação, inclusive com consequências topológicas, a qual chamamos de equivalência homotópica.

Definição 3.41 (Equivalência Homotópica) Dizemos que $X$ e $Y$ são homotopicamente equivalentes, se existem funções contínuas $f: X \rightarrow Y$ e $g: Y \rightarrow X$, tais que a composição $f \circ g$ é homotópica à identidade $i d_{Y}: Y \rightarrow Y$, enquanto $g \circ f$ é homotópica à identidade $i d_{X}: X \rightarrow X$. Neste caso, denotamos $X \simeq Y$.

Um exemplo interessante desta relação descrita acima é que $S^{1} \simeq \mathbb{R}^{2} \backslash\{(0,0)\}$ a qual veremos em detalhes no próximo exemplo.

Exemplo 3.42 Considere a identidade $i d_{S^{1}}: S^{1} \rightarrow S^{1}$, a identidade $i d_{\mathbb{R}^{2} \backslash\{(0,0)\}}: \mathbb{R}^{2} \backslash\{(0,0)\} \rightarrow \mathbb{R}^{2} \backslash\{(0,0)\}$ e as funções $f: \mathbb{R}^{2} \backslash\{(0,0)\} \rightarrow S^{1}$, dada por $f(v)=\frac{v}{|v|}$ e $g: S^{1} \rightarrow \mathbb{R}^{2} \backslash\{(0,0)\}$, definida por $g(u)=u$, as quais são claramente contínuas e ainda para $u \in S^{1}$ temos $f \circ g(u)=f(u)=u$, ou seja, $f \circ g=i d_{S^{1}} \simeq i d_{S^{1}}$. Além disso, para $v \in \mathbb{R}^{2} \backslash\{(0,0)\}$ temos $g \circ f(v)=\frac{v}{|v|} \in S^{1} \subset \mathbb{R}^{2} \backslash\{(0,0)\}$. Defina

$$
\begin{aligned}
\Gamma: \mathbb{R}^{2} \backslash\{(0,0)\} \times[0,1] & \longrightarrow \mathbb{R}^{2} \backslash\{(0,0)\} \\
(v, t) & \longmapsto t(g \circ f(v))+(1-t) i d_{\mathbb{R}^{2} \backslash\{(0,0)\}}(v)
\end{aligned}
$$

em que $\Gamma(v, t)=\frac{t}{|v|} v+(1-t) v=\left(\frac{t(1-|v|)+|v|}{|v|}\right) v$, então se tivermos $\Gamma(v, t)=(0,0) \in \mathbb{R}^{2}$, devemos ter $t(1-|v|)+|v|=0 \Rightarrow|v|=-t /(1-t) \Rightarrow$ $-t /(1-t)>0$, então não poderíamos ter $t=0$ ou $t=1$, assim, no nosso caso temos $0<t<1 \Rightarrow-1<-t<0 \Rightarrow 0<1-t<1$, o que comprova que não há possibilidade de termos $-t /(1-t)>0$, portanto para qualquer 
$v \in \mathbb{R}^{2} \backslash\{(0,0)\}$ e $t \in[0,1], \Gamma(v, t) \in \mathbb{R}^{2} \backslash\{(0,0)\}$, em que $\Gamma$ é claramente uma função contínua e é tal que $\Gamma(v, 0)=i d_{\mathbb{R}^{2} \backslash\{(0,0)\}}(v)$ e $\Gamma(v, 1)=g \circ f(v)$, o que prova $g \circ f \simeq i d_{\mathbb{R}^{2} \backslash\{(0,0)\}}$.

Podemos perceber que espaços homeomorfos são, em partícular, homotopicamente equivalentes, e uma das consequências topológicas interessantes é que quaisquer espaços topológicos homotopicamente equivalentes, possuirão grupos fundamentais isomorfos.

Definição 3.43 (Homotopia entre caminhos) Seja $\gamma_{0}, \gamma_{1}:[0,1] \rightarrow X$ caminhos tais que $\gamma_{0}(0)=\gamma_{1}(0)=x_{0}$ e $\gamma_{0}(1)=\gamma_{1}(1)=x_{1}$, em que $x_{0}, x_{1} \in X$. Dizemos que $\gamma_{0}$ e $\gamma_{1}$ são caminhos homotópicos em $X$, se existe uma função contínua $\Gamma:[0,1] \times[0,1] \rightarrow X$ tal que

$$
\begin{gathered}
\Gamma(x, 0)=\gamma_{0}(x), \Gamma(x, 1)=\gamma_{1}(x) \\
\Gamma(0, t)=x_{0}, \Gamma(1, t)=x_{1}
\end{gathered}
$$

para todo $x \in[0,1]$ e para todo $t \in[0,1]$. Chamamos $\Gamma$ de uma homotopia de caminhos entre $\gamma_{0}$ e $\gamma_{1}$, e denotamos $\gamma_{0} \simeq \gamma_{1}$.

Com respeito a caminhos homotópicos, obtemos um resultado relacionado ao conceito de índice, apresentado na seção anterior, abordado na Definição 3.20, o qual observaremos abaixo.

Observação 3.44 Se $\gamma_{0}$ e $\gamma_{1}$ são caminhos fechados homotópicos em $\Omega \subset \mathbb{C}$, então para todo $a \notin \Omega$, ind $d_{\gamma_{0}}(a)=\operatorname{ind}_{\gamma_{1}}(a)$.

Além disso, é intuitivo perceber que a noção de homotopia de caminhos em $X$ nos fornece uma relação de equivalência, a qual nos permite considerar para um caminho qualquer $\gamma:[0,1] \rightarrow X$ a sua classe de caminhos homotópicos em $X$, a qual denoratemos por $[\gamma]=\{\beta$ caminho em $X: \gamma \simeq \beta\}$. Note ainda que, a operação concatenação de caminhos, a qual definimos em 3.39, induz uma operação para as classes de caminhos homotópicos em $X$, dada por $[\gamma] *[\beta]=[\gamma * \beta]$

Observação 3.45 Apesar da operação *, definida para as classes de caminhos homotópicos em $X$, satisfazer propriedades que se assemelham aos axiomas de grupo, devemos nos atentar ao fato de que * não está definida para qualquer par de caminhos $\gamma$ e $\beta$ em $X$, consequentemente, não está definida para qualquer par de classes $[\gamma]$ e $[\beta]$. Fazendo sentido apenas no caso em que $\gamma(1)=\beta(0)$. 
Munido das noções que apresentamos até o momento, é pertinente que prossigamos para o foco desta seção, o Grupo Fundamental. Como mencionamos acima o conjunto das classes de caminhos homotópicos em $X$, munido com a operação concatenação, não forma um grupo. Contudo, ao tomarmos um ponto $x_{0} \in X$, o qual chamaremos de ponto base, e viermos a restringir as classes de caminhos homotópicos em $X$ apenas aos caminhos que começam e terminam neste ponto, isto é, todo caminho $\gamma:[0,1] \rightarrow X$ com $\gamma(0)=\gamma(1)=x_{0}$, então este conjunto munido à operação $*$ nos fornecerá um grupo, o qual definiremos formalmente a seguir.

Definição 3.46 (Grupo Fundamental) Seja $X$ um espaço e $x_{0}$ um ponto de $X$. A um caminho $\gamma:[0,1] \rightarrow X$, o qual $\gamma(0)=\gamma(1)=x_{0}$, damos o nome de loop baseado em $x_{0}$. Ao conjunto das classes de caminhos homotópicos de loops baseados em $x_{0}$, munido com a operação $*$, damos o nome de grupo fundamental de $\boldsymbol{X}$, relativo ao ponto base $x_{0}$, o qual é denotado por $\pi_{1}\left(X, x_{0}\right)$.

Um questionamento automático é nos perguntarmos em que medida o grupo fundamental depende do ponto base, assim sendo caminharemos no sentido de elucidar esta questão.

Definição 3.47 Seja $\alpha:[0,1] \rightarrow X$ um caminho em $X$ tal que $\alpha(0)=x_{0} e$ $\alpha(1)=x_{1}$. Então definimos o mapa

$$
\begin{aligned}
\hat{\alpha}: \pi_{1}\left(X, x_{0}\right) & \rightarrow \pi_{1}\left(X, x_{1}\right) \\
{[\gamma] } & \mapsto[\bar{\alpha}] *[\gamma] *[\alpha]
\end{aligned}
$$

O mapa $\hat{\alpha}$ está bem definido pois a concatenação de caminhos em $X$, operação $*$, está bem definida, já que $\bar{\alpha}(1)=x_{0}=\gamma(0)$ e $\gamma(1)=x_{1}=\alpha(0)$. Além disso, é clara a relação de que, se $\gamma$ é um loop baseado em $x_{0}$, então $\hat{\alpha} * \gamma * \alpha$ é um loop baseado em $x_{1}$. Portanto $\hat{\alpha}$ mapeia $\pi_{1}\left(X, x_{0}\right)$ em $\pi_{1}\left(X, x_{1}\right)$, como desejado.

Observação 3.48 O mapeamento $\hat{\alpha}$ depende apenas da classe de caminhos homotópicos à $\alpha$.

Teorema 3.49 O mapa $\hat{\alpha}$ é um isomorfismo de grupos.

Pelo modo como definimos o mapa $\hat{\alpha}$, nota-se que para dois grupos fundamentais de um mesmo espaço topológico $X$, relativo a pontos bases 
distintos como $\pi_{1}\left(X, x_{0}\right)$ e $\pi_{1}\left(X, x_{1}\right)$ serem isomorfos, basta que exista um caminho em $X$, que ligue os pontos $x_{0}$ e $x_{1}$. Mais geralmente, para que o grupo fundamental de um espaço $X$ independa do ponto base escolhido, basta que exista sempre um caminho que ligue quaisquer dois pontos em $X$. Claramente, é suficiente então que o espaço seja conexo por caminhos, assim segue o corolário direto do Teorema acima.

Corolário 3.50 Se X é um espaço conexo por caminhos e $x_{0}, x_{1}$ são dois pontos de $X$, então $\pi_{1}\left(X, x_{0}\right)$ é isomorfo à $\pi_{1}\left(X, x_{1}\right)$.

Consequentemente quando $X$ é conexo por caminhos, já estamos cientes de que todos os grupos fundamentais $\pi_{1}(X, x)$ são isomorfos para todo $x \in X$, então é natural estarmos tentados a tratarmos simplesmente o grupo fundamental de $X$, sem fazermos referência ao ponto base em questão. Contudo, um fato importante que deve ser comentado, é a dificuldade em obter uma maneira natural de identificar $\pi_{1}\left(X, x_{0}\right)$ com $\pi_{1}\left(X, x_{1}\right)$, pois diferentes caminhos $\alpha, \beta:[0,1] \rightarrow X$ tais que $\alpha(0)=\beta(0)=x_{0}$ e $\alpha(1)=\beta(1)=1$, podem originar isomorfismos diferentes. Por esta razão, omitir o ponto base do grupo fundamental pode nos levar ao erro.

Observação 3.51 Acontece que o isomorfismo entre $\pi_{1}\left(X, x_{0}\right)$ e $\pi_{1}\left(X, x_{1}\right)$ é independente de caminho se, e somente se, o grupo fundamental é abeliano, e portanto, neste caso faz sentido omitirmos o ponto base.

Um outro resultado relevante à respeito dos grupos fundamentais de espaços conexos por caminhos será enunciado à seguir, e pode ser encontrado com todos os devidos detalhes em [13].

Teorema 3.52 Sejam $X$ e $Y$ espaços conexos por caminhos, $x_{0} \in X$ e $y_{0} \in Y$, então $\pi_{1}\left(X \times Y,\left(x_{0}, y_{0}\right)\right)$ é isomorfo à $\pi_{1}\left(X, x_{0}\right) \times \pi_{1}\left(Y, y_{0}\right)$.

Uma outra propriedade topológia que influencia diretamente no grupo fundamental de um espaço é a simples conexidade, pois como sabemos, um espaço simplesmente conexo pode ser definido de modo que, quaisquer dois caminhos fechados neste espaço são homotópicos, o que nos fornece o resultado a seguir.

Teorema 3.53 (Grupo Fundamental Trivial) Seja X um espaço simplesmente conexo. Então, para todo $x \in X$ o grupo fundamental $\pi_{1}(X, x)$ é o que chamamos de trivial, ou seja, formado por apenas um elemento, e o denotamos por $\pi_{1}(X, x)=0$. 
Como foi mencionado anteriormente, neste capítulo estamos interessados em evidênciar o conceito de grupo fundamental, contudo, estamos particularmente interessados em explicitar o grupo fundamental dos espaços $S^{1}$, o espaço projetivo $\operatorname{PSL}(2, \mathbb{R})$ e ainda $\operatorname{PSL}(2, \mathbb{R}) \backslash\{[I]\}$, em que

$$
\operatorname{PSL}(2, \mathbb{R})=\mathrm{SL}(2, \mathbb{R}) /\{ \pm I\}=\mathrm{SL}(2, \mathbb{R}) / g \sim-g
$$

onde $\operatorname{SL}(2, \mathbb{R})=\{g \in \operatorname{Mat}(2, \mathbb{R}): \operatorname{det}(g)=1\}$ e $\sim$ é a relação de equivalência que relaciona cada $g \in \operatorname{SL}(2, \mathbb{R})$ com $-g$, sendo $\operatorname{Mat}(2, \mathbb{R})$ o espaço constituído pelas matrizes $2 \times 2$ com coeficientes reais. Sabemos que $S^{1}$ é conexo por caminhos, desta maneira, todos os grupos fundamentais $\pi_{1}\left(S^{1}, x\right)$ são isomorfos, para todo $x \in S^{1}$, além disso, o teorema a seguir é um fato relevante e bastante conhecido em Topologia Algébrica, o qual esclarece a construção do grupo fundamental de $S^{1}$, e basicamente prova que para todo $x \in S^{1}$, o grupo fundamental $\pi_{1}\left(S^{1}, x\right)$ é isomorfo ao grupo $(\mathbb{Z},+)$, o grupo aditivo dos inteiros, o qual é abeliano, e portanto, em partícular, permite nos referirmos ao grupo fundamental de $S^{1}$ apenas por $\pi_{1}\left(S^{1}\right)$, omitindo o ponto base.

Teorema 3.54 O grupo fundamental de $S^{1}$ é isomorfo ao grupo aditivo dos inteiros.

Prova. A prova deste teorema, iniciando-se por toda a construção necessária para o entendimento, pode ser encontrada com detalhes em [13], mais precisamente na página 341.

Observação 3.55 No decorrer deste trabalho, tomaremos a liberdade de tratar apenas por $\mathbb{Z}$ o grupo $(\mathbb{Z},+)$.

Dando prosseguimento, é interessante ressaltar que a construção do argumento que nos permite explicitar os grupos fundamentais desejados, se dá de forma natural, no sentido de que devemos primeiramente elucidar o grupo fundamental do espaço $\operatorname{SL}(2, \mathbb{R})$, para que em seguida possamos explicitar o grupo fundamental do espaço $\operatorname{PSL}(2, \mathbb{R})$, e então finalmente seja possível esclarecer o grupo fundamental de $\operatorname{PSL}(2, \mathbb{R}) \backslash\{[I]\}$. Começaremos utilizando o Teorema da Decomposição de Iwasawa, o qual pode ser visto em detalhes, para o caso geral, em [12], a fim de obtermos tal decomposição para $\operatorname{SL}(2, \mathbb{R})$. À esta decomposição daremos os devidos detalhes, contudo, os mesmos podem ainda ser encontrados em [5]. Para os argumentos que se seguem, não somente 
neste capítulo, estaremos considerando os espaços:

$$
\begin{aligned}
& K:=\left\{R_{\theta}=\left(\begin{array}{cc}
\cos \theta & -\sin \theta \\
\sin \theta & \cos \theta
\end{array}\right): \theta \in[0,2 \pi]\right\}=\operatorname{SO}(2, \mathbb{R}) \\
& A:=\left\{\left(\begin{array}{ll}
r & 0 \\
0 & \frac{1}{r}
\end{array}\right): r \in \mathbb{R}, r>0\right\} \\
& N:=\left\{\left(\begin{array}{ll}
1 & x \\
0 & 1
\end{array}\right): x \in \mathbb{R}\right\}
\end{aligned}
$$

Teorema 3.56 (Decomposição de Iwasawa de $S L(2, \mathbb{R})$ ) Considere os espaços definidos acima, então temos a decomposição $S L(2, \mathbb{R})=K A N$, isto é, para cada $g \in S L(2, \mathbb{R})$ existe uma única representação de $g=k a^{\prime} n$, em que $k \in K$, $a^{\prime} \in A$ e $n \in N$.

Prova. Obteremos a referida decomposição utilizando uma ação desse grupo com base em $\mathbb{R}^{2}$. Considere a base canônica de $\mathbb{R}^{2}\left\{e_{1}, e_{2}\right\}=\left\{\left(\begin{array}{l}1 \\ 0\end{array}\right),\left(\begin{array}{l}0 \\ 1\end{array}\right)\right\}$ e seja $g=\left(\begin{array}{ll}a & b \\ c & d\end{array}\right) \in \operatorname{SL}(2, \mathbb{R})$. Aplicando $g$ a base canônica obtemos $g e_{1}=\left(\begin{array}{l}a \\ c\end{array}\right)$, $g e_{2}=\left(\begin{array}{l}b \\ d\end{array}\right)$, os quais formam também uma base de $\mathbb{R}^{2}$, pois caso tivéssemos, para algum $\lambda \in \mathbb{R}, a=\lambda b$ e $c=\lambda d$, teríamos $a d-b c=0$, o que não é possível já que det $g=a d-b c=1$. O objetivo é passarmos desta nova base obtida à base canônica de $\mathbb{R}^{2}$ por meio de uma sequência de transformações em $\operatorname{SL}(2, \mathbb{R})$. Seja $\theta$ o ângulo entre o semi-eixo positivo de $x$ e $g e_{1}$, e seja $p_{\theta}$ a rotação no plano ao redor da origem, de ângulo $\theta$ e sentido anti-horário, portanto $p_{-\theta}\left(g e_{1}\right)$ está sobre o semi-eixo positivo de $x$. Como det $g$ é em particular positivo, o par ordenado de vetores $\left(g e_{1}, g e_{2}\right)$ possui mesma orientação que o par ordenado $\left(e_{1}, e_{2}\right)$, então $p_{-\theta}\left(g e_{2}\right)$ está também no semiplano superior. Como $p_{-\theta}\left(g e_{1}\right)$ está sobre o semi-eixo positivo de $x$, então é um múltiplo escalar positivo de $e_{1}$, deste modo, o normalizaremos afim de obtermos $e_{1}$. Defina $r$ por $r=\left|p_{-\theta}\left(g e_{1}\right)\right|=\left|g e_{1}\right|=\sqrt{a^{2}+c^{2}}$. Note que aplicando $\left(\begin{array}{cc}\frac{1}{r} & 0 \\ 0 & \frac{1}{r}\end{array}\right)$ à $p_{-\theta}\left(g e_{1}\right)$ nós obtemos o efeito desejado, que é $p_{-\theta}\left(g e_{1}\right) \mapsto e_{1}$, porém esta matriz não tem determinante 1 . Por outro lado, $\left(\begin{array}{cc}\frac{1}{r} & 0 \\ 0 & r\end{array}\right)$ tem determinante 1 e nos fornece o mesmo efeito desejado em $p_{-\theta}\left(g e_{1}\right)$, de fato,

$$
\left(\begin{array}{cc}
\frac{1}{r} & 0 \\
0 & r
\end{array}\right) p_{-\theta}\left(g e_{1}\right)=\left(\begin{array}{cc}
\frac{1}{r} & 0 \\
0 & r
\end{array}\right)\left(\begin{array}{l}
r \\
0
\end{array}\right)=\left(\begin{array}{l}
1 \\
0
\end{array}\right)=e_{1} .
$$


Então, esta matriz aplicada à $\mathbb{R}^{2}$ nos fornece uma aplicação que leva $p_{-\theta}\left(g e_{1}\right)$ em $e_{1}$, agora devemos ver como $p_{-\theta}\left(g e_{2}\right)$ se comporta sob esta aplicação. Sabemos que $p_{-\theta}\left(g e_{1}\right)$ está sobre o semi-eixo positivo de $x$, o determinante da matriz $\left(\begin{array}{cc}\frac{1}{r} & 0 \\ 0 & r\end{array}\right)$ é positivo e ainda que $p_{-\theta}\left(g e_{2}\right)$ está no semiplano superior, portanto podemos afirmar que $\left(\begin{array}{cc}\frac{1}{r} & 0 \\ 0 & r\end{array}\right) p_{-\theta}\left(g e_{2}\right)$ também está no semiplano superior, e assim, juntamente com $e_{1}=\left(\begin{array}{cc}\frac{1}{r} & 0 \\ 0 & r\end{array}\right) p_{-\theta}\left(g e_{1}\right)$ formam duas arestas de um paralelogramo, o qual tem área 1, já que o determinante da matriz em questão é 1 . Um paralelogramo com área 1 e tendo como base $e_{1}$ deve ter altura 1 , deste modo temos

$$
\left(\begin{array}{cc}
\frac{1}{r} & 0 \\
0 & r
\end{array}\right) p_{-\theta}\left(g e_{2}\right)=\left(\begin{array}{l}
x \\
1
\end{array}\right)
$$

para algum $x \in \mathbb{R}$, e como é sabido, a área deste paralelogramo é dado por

$$
\left|e_{1} \times\left(\begin{array}{cc}
\frac{1}{r} & 0 \\
0 & r
\end{array}\right) p_{-\theta}\left(g e_{2}\right)\right|=\operatorname{abs}\left|\begin{array}{cc}
1 & 0 \\
x & 1
\end{array}\right|=1
$$

e perceba que o resultado é inalterado para qualquer $x \in \mathbb{R}$. Qualquer transformação de cisalhamento horizontal, representado pela matriz $\left(\begin{array}{ll}1 & t \\ 0 & 1\end{array}\right)$, a qual tem determinante 1 , fixa o eixo $x$ e age nas retas horizontais de modo à esticá-las, de fato,

$$
\left(\begin{array}{ll}
1 & t \\
0 & 1
\end{array}\right) e_{1}=\left(\begin{array}{ll}
1 & t \\
0 & 1
\end{array}\right)\left(\begin{array}{l}
1 \\
0
\end{array}\right)=\left(\begin{array}{l}
1 \\
0
\end{array}\right)=e_{1}
$$

e para qualquer $y \in \mathbb{R}$ fixado temos

$$
\left(\begin{array}{ll}
1 & t \\
0 & 1
\end{array}\right)\left(\begin{array}{l}
w \\
y
\end{array}\right)=\left(\begin{array}{c}
w+t y \\
y
\end{array}\right)
$$

Assim, aplicando o cisalhamento horizontal $\left(\begin{array}{cc}1 & -x \\ 0 & 1\end{array}\right)$ à $\mathbb{R}^{2}$ tem-se

$$
\begin{gathered}
e_{1} \longmapsto\left(\begin{array}{cc}
1 & -x \\
0 & 1
\end{array}\right) e_{1}=e_{1}, \\
\left(\begin{array}{l}
x \\
1
\end{array}\right) \longmapsto\left(\begin{array}{cc}
1 & -x \\
0 & 1
\end{array}\right)\left(\begin{array}{l}
x \\
1
\end{array}\right)=\left(\begin{array}{l}
0 \\
1
\end{array}\right)=e_{2},
\end{gathered}
$$


então, nós obtemos

$$
\begin{aligned}
& e_{1} \longmapsto\left(\begin{array}{cc}
1 & -x \\
0 & 1
\end{array}\right)\left(\begin{array}{cc}
\frac{1}{r} & 0 \\
0 & r
\end{array}\right) p_{-\theta}\left(g e_{1}\right)=\left(\begin{array}{cc}
1 & -x \\
0 & 1
\end{array}\right) e_{1}=e_{1}, \\
& e_{2} \longmapsto\left(\begin{array}{cc}
1 & -x \\
0 & 1
\end{array}\right)\left(\begin{array}{cc}
\frac{1}{r} & 0 \\
0 & r
\end{array}\right) p_{-\theta}\left(g e_{2}\right)=\left(\begin{array}{cc}
1 & -x \\
0 & 1
\end{array}\right)\left(\begin{array}{l}
x \\
1
\end{array}\right)=e_{2} .
\end{aligned}
$$

Portanto, finalmente retornamos à base canônica $e_{1}, e_{2}$, partindo da base $g e_{1}, g e_{2}$, por meio de uma sequência de transformações em $\operatorname{SL}(2, \mathbb{R})$, pois a todo momento tomamos transformações cujas matrizes estão em $\operatorname{SL}(2, \mathbb{R})$, em que a composição final destas transformações é

$$
\left(\begin{array}{cc}
1 & -x \\
0 & 1
\end{array}\right)\left(\begin{array}{cc}
\frac{1}{r} & 0 \\
0 & r
\end{array}\right) p_{-\theta}, \quad \text { em que } \quad\left(\begin{array}{cc}
1 & -x \\
0 & 1
\end{array}\right)\left(\begin{array}{cc}
\frac{1}{r} & 0 \\
0 & r
\end{array}\right) p_{-\theta} g
$$

leva $e_{1}$ em $e_{1}$ e $e_{2}$ em $e_{2}$. Como sabemos, uma transformação em $\mathbb{R}^{2}$ é determinada pelo modo como age em uma base deste espaço, desta maneira, esta transformação é exatamente a identidade, e assim

$$
\left(\begin{array}{cc}
1 & -x \\
0 & 1
\end{array}\right)\left(\begin{array}{cc}
\frac{1}{r} & 0 \\
0 & r
\end{array}\right) p_{-\theta} g=\left(\begin{array}{ll}
1 & 0 \\
0 & 1
\end{array}\right)
$$

Como é sabido, a transformação $p_{-\theta}$ assume a forma matricial $R_{-\theta}$ e assim, da equação acima, obtemos

$$
\begin{aligned}
g & =\left(\begin{array}{cc}
\cos \theta & \sin \theta \\
-\sin \theta & \cos \theta
\end{array}\right)^{-1}\left(\begin{array}{cc}
\frac{1}{r} & 0 \\
0 & r
\end{array}\right)^{-1}\left(\begin{array}{cc}
1 & -x \\
0 & 1
\end{array}\right)^{-1} \\
& =\left(\begin{array}{cc}
\cos \theta & -\sin \theta \\
\sin \theta & \cos \theta
\end{array}\right)\left(\begin{array}{ll}
r & 0 \\
0 & \frac{1}{r}
\end{array}\right)\left(\begin{array}{ll}
1 & x \\
0 & 1
\end{array}\right) \in K A N
\end{aligned}
$$

A expressão de $g$ como um produto $k a^{\prime} n$ com $k \in K, a^{\prime} \in A$ e $n \in N$ caracteriza uma decomposição de Iwasawa de $g$. Devemos agora checar que tal decomposição é única para cada ângulo $\theta \in[0,2 \pi], r>0$ e $x \in \mathbb{R}$. Seja $g=\left(\begin{array}{ll}a & b \\ c & d\end{array}\right) \in \mathrm{SL}(2, \mathbb{R})$, então pela decomposição encontrada acima obtemos $g=\left(\begin{array}{ll}r \cos \theta & x r \cos \theta-\frac{1}{r} \sin \theta \\ r \sin \theta & x r \sin \theta+\frac{1}{r} \cos \theta\end{array}\right)$ e além disso, como já calculamos no decorrer da prova temos

$$
r=\sqrt{a^{2}+c^{2}}>0,
$$


já que $a d-b c=1$, portanto não podemos ter $a=c=0$. Temos ainda

$$
\begin{aligned}
& a=r \cos \theta \quad \Rightarrow \quad \cos \theta=\frac{a}{r} \\
& c=r \cos \theta \quad \Rightarrow \quad \cos \theta=\frac{c}{r}
\end{aligned}
$$

e além disso

$$
\begin{aligned}
& b=x r \cos \theta-\frac{1}{r} \sin \theta \Rightarrow x=\frac{b+\frac{1}{r} \sin \theta}{r \cos \theta}, \text { se } \cos \theta \neq 0 \\
& d=x r \sin \theta+\frac{1}{r} \cos \theta \Rightarrow x=\frac{d-\frac{1}{r} \cos \theta}{r \sin \theta}, \text { se } \sin \theta \neq 0
\end{aligned}
$$

e assim, por 3-1, 3-2 e 3-3 segue que

$$
x=\frac{b+\frac{1}{r} \frac{c}{r}}{r \frac{a}{r}}=\frac{r^{2} b+c}{a r^{2}}=\frac{a^{2} b+c(b c+1)}{a\left(a^{2}+c^{2}\right)}=\frac{a^{2} b+a c d}{a\left(a^{2}+c^{2}\right)}=\frac{a b+c d}{a^{2}+c^{2}}
$$

Note que todos os parâmetros $\theta, r$ e $x$, os quais compõem a decomposição de Iwasawa de $g=\left(\begin{array}{ll}a & b \\ c & d\end{array}\right)$, são determinados em 3-1, 3-2 e 3-4, e pelo modo como são definidos em função de $a, b, c$ e $d$, fica clara a unicidade da decomposição.

O próximo fato relevante que queremos apresentar é determinado diretamente pela decomposição de Iwasawa do grupo $\mathrm{SL}(2, \mathbb{R})$, apresentada acima, deste modo a enunciaremos como um corolário do teorema anterior.

Corolário 3.57 A aplicação

$$
\begin{aligned}
f: K \times A \times N & \longrightarrow S L(2, \mathbb{R}) \\
\left(k, a^{\prime}, n\right) & \longmapsto k a^{\prime} n
\end{aligned}
$$

é um homeomorfismo.

Prova. A função $f$ é claramente contínua, pois é o produto das projeções $\left(k, a^{\prime}, n\right) \mapsto k,\left(k, a^{\prime}, n\right) \mapsto a^{\prime}$ e $\left(k, a^{\prime}, n\right) \mapsto n$, é sobrejetiva pela decomposição de Iwasawa de $\operatorname{SL}(2, \mathbb{R})$ provada no Teorema 3.56, e ainda é injetiva pela unicidade da decomposição. Nos resta então encontrar uma inversa para $f$ e provarmos que esta é contínua. Seja $g=\left(\begin{array}{ll}a & b \\ c & d\end{array}\right) \in \operatorname{SL}(2, \mathbb{R})$ e defina as funções

$$
\begin{array}{rlrl}
r: \mathrm{SL}(2, \mathbb{R}) & \longrightarrow(0,+\infty) \quad, \quad k: \mathrm{SL}(2, \mathbb{R}) & \longrightarrow K \\
g & \longmapsto \sqrt{a^{2}+c^{2}} & g & \longmapsto\left(\begin{array}{cc}
\frac{a}{r(g)} & -\frac{c}{r(g)} \\
\frac{c}{r(g)} & \frac{a}{r(g)}
\end{array}\right)
\end{array}
$$




$$
\begin{aligned}
& a^{\prime}: \mathrm{SL}(2, \mathbb{R}) \longrightarrow A \quad e \quad n: \mathrm{SL}(2, \mathbb{R}) \longrightarrow N \\
& g \longmapsto\left(\begin{array}{cc}
r(g) & 0 \\
0 & \frac{1}{r(g)}
\end{array}\right) \quad g \longmapsto\left(\begin{array}{cc}
1 & \frac{a b+c d}{a^{2}+c^{2}} \\
0 & 1
\end{array}\right)
\end{aligned}
$$

Vale ressaltar, com base nos cálculos feitos no fim da prova do Teorema 3.56, tais funções fazem sentido e estão bem definidas, e como vimos temos $g=k(g) a^{\prime}(g) n(g)$ de forma única. Agora, defina a função

$$
\begin{aligned}
h: \mathrm{SL}(2, \mathbb{R}) & \longrightarrow K \times A \times N \\
g & \longmapsto\left(k(g), a^{\prime}(g), n(g)\right),
\end{aligned}
$$

em que $f \circ h(g)=f\left(\left(k(g), a^{\prime}(g), n(g)\right)\right)=k(g) a^{\prime}(g) n(g)=g$ e $h \circ f\left(\left(k(g), a^{\prime}(g), n(g)\right)\right)=h\left(k(g) a^{\prime}(g) n(g)\right)=\left(k(g), a^{\prime}(g), n(g)\right)$. Então $h$ é uma inversa de $f$, além disso é uma função contínua. De fato, considere $\left(g_{j}\right)_{j \in \mathbb{N}}=\left(\begin{array}{ll}a_{j} & b_{j} \\ c_{j} & d_{j}\end{array}\right) \in \mathrm{SL}(2, \mathbb{R})$ tal que $g_{j} \rightarrow g=\left(\begin{array}{ll}a & b \\ c & d\end{array}\right) \in \mathrm{SL}(2, \mathbb{R})$, assim temos $a_{j} \rightarrow a, b_{j} \rightarrow b, c_{j} \rightarrow c$ e $d_{j} \rightarrow d$, quando $j \rightarrow \infty$, consequentemente temos $k\left(g_{j}\right) \rightarrow k(g), a^{\prime}\left(g_{j}\right) \rightarrow a^{\prime}(g)$ e $n\left(g_{j}\right) \rightarrow n(g)$, já que as funções $k, a^{\prime}$ e $n$ são definidas exclusivamente em função dos coeficientes da matriz avaliada.

Além do homeomorfismo apresentado acima, este resultado nos permite evidenciar outros homeomorfismos claros, porém relevantes, entre os espaços $K, A, N$, e espaços que fazem parte do nosso cotidiano. São eles

$$
\begin{array}{rlrl}
K \longrightarrow[0,2 \pi] \longrightarrow S^{1} & \quad S^{1} \longrightarrow[0,2 \pi] & \longrightarrow K \\
R_{\theta} \longmapsto \theta & e^{i \theta} \longmapsto \theta & \longmapsto & \longmapsto R_{\theta}
\end{array}
$$

em que consideramos $[0,2 \pi]$ com 0 e $2 \pi$ identificados, que na realidade se trata de $\mathbb{R} / x \sim x+2 \pi$, a reta real quocientada pela relação que identifica $x \in \mathbb{R}$ e $x+2 \pi \in \mathbb{R}$, e tomamos sempre o representante de cada clase em $[0,2 \pi)$, o que prova que os espaços $S^{1}$ e $K$ são homeomorfos. Temos também,

$$
\begin{aligned}
& A \longrightarrow(0,+\infty) \longrightarrow \mathbb{R} \quad, \quad \mathbb{R} \longrightarrow(0,+\infty) \longrightarrow A \\
& \left(\begin{array}{cc}
r & 0 \\
0 & \frac{1}{r}
\end{array}\right) \longmapsto r \quad r \quad t \longmapsto \log r \quad e^{t} \longmapsto\left(\begin{array}{cc}
e^{t} & 0 \\
0 & \frac{1}{e^{t}}
\end{array}\right)
\end{aligned}
$$

comprovando o fato de $A$ e $\mathbb{R}$ serem espaços homeomorfos, e ainda, talvez o mais claro, seja o fato de $N$ ser homeomorfo à $\mathbb{R}$. Deste modo, temos $\operatorname{SL}(2, \mathbb{R})$ homeomorfo à $K \times A \times N$, o qual é homeomorfo à $S^{1} \times \mathbb{R} \times \mathbb{R}$, assim $\operatorname{SL}(2, \mathbb{R})$ acaba por ser homeomorfo à $S^{1} \times \mathbb{R}^{2}$. 
Observação 3.58 Como já foi definido anteriormente, quando temos caminhos homotópicos, ou ainda espaços homotopicamente equivalentes, denotamos por $\simeq$. Apenas a cargo de informação, para o futuro e quando pertinente, utilizaremos a notação $\cong$, quando nos referirmos à espaços homeomorfos ou ainda grupos isomorfos.

Portanto, já que $\mathrm{SL}(2, \mathbb{R}) \cong S^{1} \times \mathbb{R}^{2}$, sendo $S^{1}$ e $\mathbb{R}^{2}$ espaços conexos por caminhos, o que faz de $S^{1} \times \mathbb{R}^{2}$ um espaço conexo por caminhos, temos que $\operatorname{SL}(2, \mathbb{R})$ é também um espaço conexo por caminhos. Deste modo, como no caso do círculo unitário, o grupo fundamental destes espaços, relativo a um ponto base dado, é isomorfo ao grupo fundamental deste espaço relativo a qualquer outro ponto base. Contudo, como apresentado no Teorema 3.54 temos $\pi_{1}\left(S^{1}\right) \cong \mathbb{Z}$, e já que $\mathbb{R}^{2}$ é simplesmente conexo, pelo Teorema 3.53 , temos $\pi_{1}\left(\mathbb{R}^{2}\right)=0$, assim pelo Teorema 3.52 e assumindo que o homeomorfismo entre $\operatorname{SL}(2, \mathbb{R})$ e $S^{1} \times \mathbb{R}^{2}$ é tal que leva $\operatorname{SL}(2, \mathbb{R}) \ni x \mapsto\left(x_{1}, x_{2}\right) \in S^{1} \times \mathbb{R}^{2}$ temos

$$
\pi_{1}(\mathrm{SL}(2, \mathbb{R}), x) \cong \pi_{1}\left(S^{1} \times \mathbb{R}^{2},\left(x_{1}, x_{2}\right)\right) \cong \pi_{1}\left(S^{1}, x_{1}\right) \times \pi_{1}\left(\mathbb{R}^{2}, x_{2}\right) \cong \mathbb{Z} \times 0 \cong \mathbb{Z}
$$

e já que $\mathbb{Z}$ é abeliano, faz sentido omitirmos o ponto base e escrevermos

$$
\pi_{1}(\mathrm{SL}(2, \mathbb{R})) \cong \mathbb{Z}
$$

Agora, como estamos interessados em trabalhar também com os espaços $\operatorname{PSL}(2, \mathbb{R})$ e $\operatorname{PSL}(2, \mathbb{R}) \backslash\{[I]\}$, caminharemos neste sentido, de modo a começarmos quocientando o espaço $\mathrm{SL}(2, \mathbb{R})$ pela relação de equivalência $\sim$, a qual identifica as matrizes $g$ e $-g$ em $\operatorname{SL}(2, \mathbb{R})$, uma vez que $\operatorname{PSL}(2, \mathbb{R})=\operatorname{SL}(2, \mathbb{R}) /_{g \sim-g}$, e então estaremos interessados em esclarecer o efeito causado por essa quocientação no espaço homeomorfo à $\operatorname{SL}(2, \mathbb{R})$, o espaço $S^{1} \times \mathbb{R}^{2}$. Seja $g=\left(\begin{array}{ll}a & b \\ c & d\end{array}\right) \in \mathrm{SL}(2, \mathbb{R})$, então como apresentado, existe uma decomposição única da matriz $g$, na forma $g=R_{\theta} a^{\prime} n$, em que as matrizes $a^{\prime}$ e $n$ são dadas por $a^{\prime}=\left(\begin{array}{cc}r & 0 \\ 0 & \frac{1}{r}\end{array}\right)$ e $n=\left(\begin{array}{ll}1 & x \\ 0 & 1\end{array}\right)$, sendo $r=\sqrt{a^{2}+c^{2}}$, $x=\frac{a b+c d}{a^{2}+c^{2}}$ e $\cos \theta=\frac{a}{r}, \sin \theta=\frac{c}{r}$, o que implica em $\theta=\arccos \frac{a}{r}=\arcsin \frac{c}{r}$. Além disso, como vimos, temos o homeomorfismo

$$
\begin{aligned}
\mathrm{SL}(2, \mathbb{R}) & \longrightarrow K \times A \times N \longrightarrow[0,2 \pi] \times(0,+\infty) \times \mathbb{R} \longrightarrow S^{1} \times \mathbb{R}^{2} \\
g & \longmapsto\left(R_{\theta}, a^{\prime}, n\right) \\
& \longmapsto(\theta, r, x) \longmapsto\left(e^{i \theta}, \log r, x\right)
\end{aligned}
$$

Portanto, note que identificando $g$ com $-g$, estamos identificando $g$ com $-g=-\left(R_{\theta} a^{\prime} n\right)=\left(\left(-R_{\theta}\right) a^{\prime} n\right)=R_{\theta+\pi} a^{\prime} n$, isto é, acabamos por identificar 
$R_{\theta}$ com $-R_{\theta}=R_{\theta+\pi}$, que equivale a identificarmos $\theta \operatorname{com} \theta+\pi$ e consequentemente, identificarmos $e^{i \theta} \operatorname{com} e^{i(\theta+\pi)}=-e^{i \theta}$. Deste modo, a relação $g \sim-g$ causa efeito apenas sobre $S^{1}$, de modo que quocientar $\mathrm{SL}(2, \mathbb{R})$ pela relação que identifica as matrizes $g$ e $-g$ em $\mathrm{SL}(2, \mathbb{R})$, equivale a identificar os pontos antipodais em $S^{1}$, ou seja, equivale a quocientarmos $S^{1}$ pela relação $\sim$, que identifica $z$ com $-z$ em $S^{1}$, a qual denotaremos por $z \sim-z$, causando o seguinte efeito nas aplicações acima

$$
\begin{aligned}
\operatorname{PSL}(2, \mathbb{R}) & \rightarrow K / R_{\theta} \sim R_{\theta+\pi} \times A \times N \rightarrow[0,2 \pi] / \sim \times(0,+\infty) \times \mathbb{R} \rightarrow S^{1} / z \sim-z \times \mathbb{R}^{2} \\
{[g] } & \longmapsto\left(\left[R_{\theta}\right], a^{\prime}, n\right) \\
\longmapsto \quad([\theta], r, x) & \longmapsto\left(\left[e^{i \theta}\right], \log r, x\right)
\end{aligned}
$$

Como para a inversa é nítido que temos exatamente o mesmo cálculo, segue que a relação de equivalência causa o referido efeito sobre o homeomorfismo apresentado acima, de modo a obtermos

$$
\operatorname{PSL}(2, \mathbb{R})=\operatorname{SL}(2, \mathbb{R}) /_{g \sim-g} \cong S^{1} /_{z \sim-z} \times \mathbb{R}^{2}
$$

Agora, para que possamos explicitar o grupo fundamental de $\operatorname{PSL}(2, \mathbb{R})$, e assim dar prosseguimento aos objetivos planejados, precisaremos mostrar algumas propriedades à respeito do espaço $S^{1} / z \sim-z$. Assim como anteriormente, estaremos interpretando $S^{1}$ como sendo o círculo unitário no plano complexo e $\sim$ como a relação que identifica $z$ e $-z$ em $S^{1}$, ou seja, $z_{1}, z_{2} \in S^{1}$ são tais que $z_{1} \sim z_{2}$ se, e somente se, $z_{1}= \pm z_{2}$. Além disso, assim como no restante do trabalho apresentado, quando lidarmos com espaços quocientes, estaremos considerando a topologia quociente. Começaremos provando a seguinte propriedade topológica a respeito de $S^{1} / z \sim-z$.

Teorema $3.59 S^{1} / z \sim-z$ é um espaço de Hausdorff.

Prova. Considere por $\pi: S^{1} \rightarrow S^{1} / z \sim-z$ o mapa quociente canônico. Sejam $[x],[y]$ pontos distintos em $S^{1} / z \sim-z$, então os conjuntos $\pi^{-1}([x])=\{x,-x\}$ e $\pi^{-1}([y])=\{y,-y\}$ são subconjuntos disjuntos de $S^{1}$. Seja $d$ a métrica usual em $S^{1}$, então temos $d(x, y)=d(-x,-y)$ e $d(x,-y)=d(-x, y)$, fato este que geometricamente se torna ainda mais claro. Tome $r=\min \{d(x, y), d(x,-y)\}$ e defina $U_{x}=\left\{x^{\prime} \in S^{1}: d\left(x, x^{\prime}\right)<\frac{r}{2}\right\}$ e $U_{y}=\left\{y^{\prime} \in S^{1}: d\left(y, y^{\prime}\right)<\frac{r}{2}\right\}$, que são vizinhanças abertas de $x$ e $y$ respectivamente, o que é claro, pois se tratam de arcos abertos, de comprimento $r$, centrados em $x$ e $y$, respectivamente. Além disso são disjuntos, pois caso existisse $z \in U_{x} \cap U_{y}$ teríamos que $d(x, y) \leq d(x, z)+d(z, y)<r$, o que contrariaria a definição de $r$. Temos 
$\pi\left(U_{x}\right)=\left\{\left[x^{\prime}\right]: x^{\prime} \in U_{x}\right\}$ e portanto

$$
\pi^{-1}\left(\pi\left(U_{x}\right)\right)=\left\{z \in S^{1}: \pi(z) \in \pi\left(U_{x}\right)\right\}=U_{x} \cup-U_{x},
$$

em que $-U_{x}=\left\{-x^{\prime}: x^{\prime} \in U_{x}\right\}$. Portanto $\pi^{-1}\left(\pi\left(U_{x}\right)\right)$ é um aberto em $S^{1}$, já que $U_{x}$ e $-U_{x}$ são abertos em $S^{1}$, e então, pela continuidade de $\pi$, segue que $\pi\left(U_{x}\right)$ é um aberto de $S^{1} / z \sim-z$, consequentemente é uma vizinhança aberta de $[x]$. Analogamente $\pi\left(U_{y}\right)$ é uma vizinhança aberta de $[y] \mathrm{em} S^{1} /_{z \sim-z}$. Por fim, temos que $\pi\left(U_{x}\right) \cap \pi\left(U_{y}\right)=\emptyset$, já que

$$
\begin{aligned}
\pi^{-1}\left(\pi\left(U_{x}\right) \cap \pi\left(U_{y}\right)\right) & =\pi^{-1}\left(\pi\left(U_{x}\right)\right) \cap \pi^{-1}\left(\pi\left(U_{y}\right)\right)=\left(U_{x} \cup-U_{x}\right) \cap\left(U_{y} \cup-U_{y}\right) \\
& =\left(U_{x} \cap U_{y}\right) \cup\left(U_{x} \cap-U_{y}\right) \cup\left(-U_{x} \cap U_{y}\right) \cup\left(-U_{x} \cap-U_{y}\right) \\
& =\emptyset .
\end{aligned}
$$

O principal resultado que queremos obter a respeito do espaço $S^{1} / z \sim-z$ é o fato deste ser homeomorfo à $S^{1}$, contudo, para que possamos prová-lo, precisaremos de dois resultados conhecidos de topologia.

Teorema 3.60 (Propriedade Universal da Topologia Quociente) Seja $\sim$ uma relação de equivalência em $X$. Se $s: X \rightarrow Y$ é uma função contínua tal que $a \sim b$ implica $s(a)=s(b)$, para todo $a, b \in X$, então existe uma única função contínua $h: X / \sim \rightarrow Y$ tal que $s=h \circ \pi$.

Teorema 3.61 Seja $h: X \rightarrow Y$ uma função contínua e bijetiva. Se $X$ é um espaço compacto e $Y$ é um espaço de Hausdorff, então h é um homeomorfismo.

Agora estamos munidos com os resultados necessários para provar o resultado que, de fato, queremos.

Teorema 3.62 O espaço $S^{1} / z \sim-z$ é homeomorfo à $S^{1}$.

Prova. Considere a aplicação $s: S^{1} \longrightarrow S^{1}$, definida por $z \longmapsto z^{2}$. Como para $z, z^{\prime} \in S^{1}$ temos $z \sim z^{\prime}$ se, e somente se, $z= \pm z^{\prime}$, temos $s(z)=s\left(z^{\prime}\right)$, em que $s$ é claramente contínua e sobrejetiva. Portanto, pela Propriedade Universal da Topologia Quociente, Teorema 3.60, s induz uma única aplicação contínua $h: S^{1} /_{z \sim-z} \rightarrow S^{1}$ tal que $h \circ \pi=s$, em que $\pi: S^{1} \rightarrow S^{1} / z \sim-z$ é o mapa quociente canônico. Assim, para $z \in S^{1}$ temos $z^{2}=s(z)=h(\pi(z))=h([z])$. Como $s$ é sobrejetiva e $\pi$ é sobrejetiva, temos que $h$ é também sobrejetiva, além disso, para $z, z^{\prime} \in S^{1}$ quaisquer

$$
h([z])=h\left(\left[z^{\prime}\right]\right) \Longleftrightarrow z^{2}=z^{\prime 2} \Longleftrightarrow z= \pm z^{\prime} \Longleftrightarrow z \sim z^{\prime} \Longleftrightarrow[z]=\left[z^{\prime}\right]
$$


Portanto, $h$ é injetiva e consequentemente bijetiva, então $h$ é uma bijeção contínua entre entre os espaços de Hausdorff compactos, $S^{1}$ e $S^{1} / z \sim-z$. Logo, como visto no Teorema 3.61, $h$ é um homeomorfismo entre estes espaços.

Portanto, munido a este novo fato que provamos, obtemos o homeomorfismo

$$
\begin{aligned}
& \operatorname{PSL}(2, \mathbb{R}) \longrightarrow K /_{R_{\theta} \sim R_{\theta+\pi}} \times A \times N \longrightarrow S^{1} /_{z \sim-z} \times \mathbb{R}^{2} \longrightarrow S^{1} \times \mathbb{R}^{2} \\
& {[g] \longmapsto\left(\left[R_{\theta}\right], a^{\prime}, n\right) \quad \longmapsto\left(\left[e^{i \theta}\right], \log r, x\right) \longmapsto\left(e^{2 i \theta}, \log r, x\right)}
\end{aligned}
$$

Assim, pelo mesmo raciocínio apresentado anteriormente, quando evidenciamos o grupo fundamental de $\operatorname{SL}(2, \mathbb{R})$ temos

$$
\begin{aligned}
\pi_{1}(\operatorname{PSL}(2, \mathbb{R}),[g]) & \cong \pi_{1}\left(S^{1} \times \mathbb{R}^{2},\left(\left[e^{i \theta}\right], \log r, x\right)\right) \\
& \cong \pi_{1}\left(S^{1},\left[e^{2 i \theta}\right]\right) \times \pi_{1}\left(\mathbb{R}^{2},(\log r, x)\right) \cong \mathbb{Z} \times 0 \cong \mathbb{Z}
\end{aligned}
$$

e portanto, faz sentido denotarmos $\pi_{1}(\operatorname{PSL}(2, \mathbb{R})) \cong \mathbb{Z}$. Mais ainda, pelos fatos apresentados, no caso em que $g=\left(\begin{array}{ll}a & b \\ c & d\end{array}\right)=I$, matriz identidade em $\operatorname{Mat}(2, \mathbb{R})$ temos $a=d=1$ e $c=d=0$, deste modo, segue que $r=\sqrt{a^{2}+c^{2}}=1, x=\frac{a b+c d}{a^{2}+c^{2}}=1$, e $\theta=0$, então o homeomorfismo apresentado é tal que $\operatorname{PSL}(2, \mathbb{R}) \ni[I] \mapsto\left(e^{0}, \log 1,1\right)=(1,0,1) \in S^{1} \times \mathbb{R}^{2}$. Desta maneira, removermos a classe $[I]$ do espaço $\operatorname{PSL}(2, \mathbb{R})$, equivale a removermos o ponto $(1,0,1)$ do espaço $S^{1} \times \mathbb{R}^{2}$. Por outro lado, $\left(S^{1} \times \mathbb{R}^{2}\right) \backslash\{(1,0,1)\}$ é homeomorfo à $\left(S^{1} \backslash\{1\}\right) \times\left(\mathbb{R}^{2} \backslash\{(0,1)\}\right)$, em que é claro pelo que apresentamos $S^{1} \backslash\{1\} \cong(0,2 \pi) \cong \mathbb{R}$, pois qualquer intervalo aberto é homeomorfo à reta real $\mathbb{R}$, além disso, é sábido que $\mathbb{R}^{2} \backslash\{(1,0)\} \cong \mathbb{R}^{2} \backslash\left\{\left(p_{1}, p_{2}\right)\right\}$ para quaisquer $\left(p_{1}, p_{2}\right) \in \mathbb{R}^{2}$, em partícular para $p_{1}=p_{2}=0$. Deste modo, temos que os seguintes espaços são homeomorfos

$$
\operatorname{PSL}(2, \mathbb{R}) \backslash\{[I]\} \cong \mathbb{R} \times \mathbb{R}^{2} \backslash\{(0,0)\}
$$

o que mostra ainda que temos $\operatorname{PSL}(2, \mathbb{R}) \backslash\{[I]\}$ conexo por caminhos. Além disto, um outro fato fundamental apresentado no Exemplo 3.42, é que os espaços $S^{1}$ e $\mathbb{R}^{2} \backslash\{(0,0)\}$ são homotopicamente equivalentes, deste modo, $\pi_{1}\left(\mathbb{R}^{2} \backslash\{(0,0)\}\right) \cong \pi_{1}\left(S^{1}\right) \cong \mathbb{Z}$, e como $\mathbb{R}$ é simplesmente conexo seu grupo fundamental é o trivial. Desta forma, pelo mesmo racicínio já apresentado faz sentido omitirmos o ponto base e então obtermos

$$
\pi_{1}(\operatorname{PSL}(2, \mathbb{R}) \backslash\{[I]\}) \cong \pi_{1}(\mathbb{R}) \times \pi_{1}\left(\mathbb{R}^{2} \backslash\{(0,0)\}\right) \cong 0 \times \mathbb{Z} \cong \mathbb{Z}
$$


Um outro conceito relevante, particularmente para nós neste trabalho, é o de homomorfismo induzido por uma função contínua. Seja $h: X \rightarrow Y$ uma função contínua que leva $x_{0} \in X$ em $y_{0} \in Y$, a qual costumamos denotar por $h:\left(X, x_{0}\right) \rightarrow\left(Y, y_{0}\right)$. Note que, se $\gamma:[0,1] \rightarrow X$ é um loop em $X$, baseado em $x_{0}$, então $h \circ \gamma:[0,1] \rightarrow Y$ é um loop em $Y$ baseado em $y_{0}$, de fato, temos $h(\gamma(0))=h\left(x_{0}\right)=y_{0}=h\left(x_{0}\right)=h(\gamma(1))$. Deste modo, a correspondência $\gamma \mapsto h \circ \gamma$, que toma loops em $X$ baseados em $x_{0}$, ou seja, elementos de alguma classe em $\pi_{1}\left(X, x_{0}\right)$, e leva em loops em $Y$ baseados em $y_{0}$, os quais são elementos de alguma classe em $\pi_{1}\left(Y, y_{0}\right)$, dá origem à uma função que estabelece uma correspondência entre $\pi_{1}\left(X, x_{0}\right)$ e $\pi_{1}\left(Y, y_{0}\right)$.

Definição 3.63 (Homomorfismo induzido) Seja $h:\left(X, x_{0}\right) \rightarrow\left(Y, y_{0}\right)$ uma função contínua como descrevemos acima. Defina

$$
\begin{gathered}
h_{\#}: \pi_{1}\left(X, x_{0}\right) \rightarrow \pi_{1}\left(Y, y_{0}\right) \\
{[\gamma] \mapsto[h \circ \gamma]}
\end{gathered}
$$

A esta função $h_{\#}$ damos o nome de homomorfismo induzido por $h$, baseado em $x_{0}$.

Observação 3.64 O fato de $h_{\#}$ ser um homomorfismo segue da equação

$$
(h \circ \gamma) *(h \circ \beta)=h \circ(\gamma * \beta)
$$

Para encerrarmos esta seção, e à vista disso também o capítulo, apresentaremos um último tema relacionado à Topologia Algébrica, que são as funções circulares, e ainda alguns conceitos relacionados a estas. Uma função circular trata-se de uma função contínua definida do círculo unitário para o próprio círculo unitário. O primeiro conceito que trataremos é a noção de grau, que assim como grande parte dos demais resultados a respeito destas funções tratados aqui, podem ser encontrados com mais detalhes em [3]. Assim como temos feito até o momento, enunciaremos e apresentaremos resultados da maneira a qual se torna mais útil para o andamento deste trabalho. Embora estejamos focados em $S^{1}$, vale ressaltar que os resultados seguem para todo espaço $X$ homeomorfo à $S^{1}$. A noção de grau, a qual pode ser definida de diversas maneiras distintas, representará uma medição da quantidade de vezes que uma função circular envolve, cobre, o círculo unitário em torno de si mesmo, de modo que, intuitivamente, esperamos que a função $p_{1}\left(e^{i \theta}\right)=e^{i \theta}$ tenha grau 1 , assim como $p_{-1}\left(e^{i \theta}\right)=e^{-i \theta}$ tenha grau -1 , e de um modo geral, para $n \in \mathbb{Z}, p_{n}\left(e^{i \theta}\right)=e^{i n \theta}$ tenha grau $n$. Enunciaremos algumas definições para este conceito, começando pelo seguinte teorema: 
Teorema 3.65 Para cada função circular $f: S^{1} \rightarrow S^{1}$, existe um único $n \in \mathbb{Z}$ tal que $f$ é homotópica à $p_{n}: S^{1} \rightarrow S^{1}$, dada por $p_{n}\left(e^{i \theta}\right)=e^{i n \theta}$.

A partir do teorema anterior, definimos então o grau de uma função circular.

Teorema 3.66 (Grau de uma função circular) $O$ único $n \in \mathbb{N}$ associado à $f: S^{1} \rightarrow S^{1}$ no teorema anterior, Teorema 3.65, é definido como grau de $f$, e é denotado por grau $(f)$.

Observação 3.67 Dadas duas funções circulares $f, g: S^{1} \rightarrow S^{1}$, temos $\operatorname{grau}(g \circ f)=\operatorname{grau}(g) \operatorname{grau}(f)$.

Pode-se perceber que, como esperávamos, para todo $n \in \mathbb{Z}$ a função $p_{n}\left(e^{i \theta}\right)=e^{i n \theta}$ tem grau $n$, isto é, o grau de uma função circular $f: S^{1} \rightarrow S^{1}$ representa o número de vezes em que $f\left(S^{1}\right)$ cobre $S^{1}$, com referencial positivo para o sentido anti-horário. O próximo resultado nos fornecerá uma importante propriedade à respeito de funções circulares homotópicas, nos indicando que o grau determina as classes de homotopia de funções circulares.

Teorema 3.68 Sejam $f, g: S^{1} \rightarrow S^{1}$ duas funções circulares. Então $f$ e $g$ são homotópicas se, e somente se, grau $(f)=\operatorname{grau}(g)$.

Um fato relevante é que, deste modo, a menos de homotopia, os loops são classificados pelo seu grau, no sentido de que um loop $\gamma: S^{1} \rightarrow S^{1}$ cobre o círculo $n \in \mathbb{Z}$ vezes se, e somente se, sua classe $[\gamma] \in \pi_{1}\left(S_{1}\right)$ representa $n \in \mathbb{Z} \cong \pi_{1}\left(S^{1}\right)$ se, e somente se, $\gamma$ é homotópica a função $z \mapsto z^{n}$, e esta representação nos permite exibir uma definição equivalente para o conceito de grau de funções circulares.

Definição 3.69 (Grau de uma função circular) Considere uma função circular $f: S^{1} \rightarrow S^{1}$, sabemos que para $z \in S^{1}$ esta induz um homomorfismo

$$
\begin{aligned}
f_{\#}: \pi_{1}\left(S^{1}, z\right) & \longrightarrow \pi_{1}\left(S^{1}, f(z)\right) \\
{[\gamma] } & \longmapsto[f \circ \gamma],
\end{aligned}
$$

em que existe $n \in \mathbb{Z}$ tal que o homomorfismo acima pode ser interpretado como

$$
\begin{aligned}
f_{\#}: \pi_{1}\left(S^{1}, z\right) & \longrightarrow \pi_{1}\left(S^{1}, f(z)\right) \\
{[\gamma] } & \longmapsto n[\gamma],
\end{aligned}
$$


e como $\pi_{1}\left(S^{1}, z\right) \cong \mathbb{Z} \cong \pi_{1}\left(S^{1}, f(z)\right)$, pelo que discutimos, grosso modo, pode ser interpretado como

$$
\begin{aligned}
f_{\#}: \mathbb{Z} & \longrightarrow \mathbb{Z} \\
k & \longmapsto n k .
\end{aligned}
$$

$A$ este $n$ denonimados o grau de $f$.

Seguiremos exibindo outra maneira de definirmos este conceito, a qual também será útil para o desenvolvimento deste trabalho. Contudo, antes precisamos enunciar brevemente o simples conceito de levantamento para estas funções. É sabido que para uma função circular $f: S^{1} \rightarrow S^{1}$ existe um levantamento contínuo $\tilde{f}: \mathbb{R} \rightarrow \mathbb{R}$, de modo que para a projeção natural $\pi: \mathbb{R} \rightarrow S^{1}$, definida por $\pi(x)=e^{2 \pi i x}$, temos $f \circ \pi=\pi \circ \tilde{f}$, nos possibilitando fornecer a seguinte definição.

Definição 3.70 (Grau de uma função circular) Seja $f: S^{1} \rightarrow S^{1}$ uma função circular e $\tilde{f}: \mathbb{R} \rightarrow \mathbb{R}$ um levantamento, então

$$
\operatorname{grau}(f)=\tilde{f}(x+1)-\tilde{f}(x)
$$

para qualquer $x \in \mathbb{R}$.

Perceba que a definição acima, de fato, não depende do $x \in \mathbb{R}$ tomado, pois a função $\mathbb{R} \ni x \mapsto \tilde{f}(x+1)-\tilde{f}(x) \in 2 \pi \mathbb{Z}$ é contínua e $2 \pi \mathbb{Z}$ discreto, portanto é uma função constante.

Agora, considere uma função circular $f: S^{1} \rightarrow S^{1}$, note que esta induz naturalmente uma função em $[0,2 \pi]$, ou de um modo geral sobre qualquer intervalo fechado de comprimento $2 \pi$, definida da seguinte maneira

$$
\begin{aligned}
\gamma_{f}:[0,2 \pi] & \longrightarrow S^{1} \\
\theta & \longmapsto f\left(e^{i \theta}\right) .
\end{aligned}
$$

Além disso, note que $\gamma_{f}$ é um caminho fechado em $S^{1}$, pois $\gamma_{f}(0)=\gamma_{f}(2 \pi)$ e a sua continuidade, assim como o fato de estar sempre sobre o círculo unitário, seguem diretamente por $f$. Pois bem, o fato interessante a respeito das funções circulares o qual estamos interessados, se dá quando temos uma função $f: S^{1} \rightarrow S^{1}$ ao menos suave por partes, pois assim $\gamma_{f}$ também será suave por partes e obtemos então uma relação entre o grau desta função circular e o índice deste caminho fechado $\gamma_{f}$, obtido à partir de $f$, em torno da origem $z=0$. 
Teorema 3.71 Considere uma função circular suave por partes $f: S^{1} \rightarrow S^{1}$ e $\gamma_{f}:[0,2 \pi] \rightarrow S^{1}$ o caminho fechado induzido por $f$, então

$$
\operatorname{grau}(f)=\operatorname{ind}_{\gamma_{f}}(0)
$$

Prova. Por definição existe um único $n \in \mathbb{Z}$ tal que $f \simeq p_{n}$, isto é, $\operatorname{grau}(f)=n=\operatorname{grau}\left(p_{n}\right)$. Por outro lado, note que $p_{n}\left(e^{i \theta}\right)=e^{i n \theta}$ induz o caminho fechado em $S^{1}$

$$
\begin{aligned}
\gamma_{p_{n}}:[0,2 \pi] & \longrightarrow S^{1} \\
\theta & \longmapsto p_{n}\left(e^{i \theta}\right)=e^{i n \theta}
\end{aligned}
$$

e pela definição de índice, apresentada em 3.20, temos

$$
\operatorname{ind}_{\gamma_{p_{n}}}(0)=\frac{1}{2 \pi i} \int_{\gamma_{p_{n}}} \frac{1}{\xi-0} d \xi=\frac{1}{2 \pi i} \int_{0}^{2 \pi} \frac{1}{e^{i n \theta}} i n e^{i n \theta} d \theta=\frac{1}{2 \pi} \int_{0}^{2 \pi} n d \theta=n .
$$

Além disso, como $f \simeq p_{n}$, naturalmente temos que $\gamma_{f} \simeq \gamma_{p_{n}}$, pois sendo $\Gamma: S^{1} \times[0,1] \rightarrow S^{1}$ uma homotopia entre $f$ e $p_{n}$ e $\Theta:[0,2 \pi] \rightarrow S^{1}$ tal que $\Theta(\theta)=e^{i \theta}$, temos que $\tilde{\Gamma}:[0,2 \pi] \times[0,1] \rightarrow S^{1}$, definida por $\tilde{\Gamma}(\theta, t):=\Gamma(\Theta(\theta), t)$, será uma homotopia entre os caminhos $\gamma_{f}, \gamma_{p_{n}}$. Portanto pela Observação 3.44 temos $\operatorname{ind} \gamma_{f}(0)=\operatorname{ind}_{\gamma_{p_{n}}}(0)=n$, o que implica $\operatorname{ind}_{\gamma_{f}}(0)=n$. Logo $\operatorname{grau}(f)=\operatorname{ind}_{\gamma_{f}}(0)$. 
4

A demonstração da Fórmula de Avila-Bochi-Herman

Neste momento, como o título sugere, o capítulo destina-se ao exercício de elucidar em detalhes a prova da Fórmula de Avila-Bochi-Herman, a qual nos diz que para uma transformação ergódica $T: X \rightarrow X$ e um cociclo $A: X \rightarrow \mathrm{SL}(2, \mathbb{R})$, tal que $\log \|A\| \in L^{1}(\mu, X)$, tem-se

$$
\frac{1}{2 \pi} \int_{0}^{2 \pi} L\left(A R_{\theta}\right) d \theta=\int_{X} \log \left(\frac{\|A(x)\|+\|A(x)\|^{-1}}{2}\right) d \mu(x) .
$$

No entanto, este Teorema será uma consequência simples do resultado determinístico, que nos permite afimar para $A_{1}, \ldots, A_{n} \in \mathrm{SL}(2, \mathbb{R})$ a seguinte igualdade

$$
\frac{1}{2 \pi} \int_{0}^{2 \pi} \log \rho\left(A_{n} R_{\theta} \ldots A_{1} R_{\theta}\right) d \theta=\sum_{j=1}^{n} N\left(A_{j}\right)
$$

em que a ideia principal de sua demonstração consiste na complexificação da matriz $A_{n} R_{\theta} \ldots A_{1} R_{\theta}$, de modo a obtermos uma matriz complexa $C_{z}$, holomorfa. Acontece que, e na verdade grande parte do texto se destinará a este objetivo, existe uma parametrização holomorfa do espectro dessa matriz $C_{z}$. Mais precisamente, lembre-se que no capítulo 2 definimos as matrizes

$$
S_{z}=\left(\begin{array}{cc}
\frac{z+z^{-1}}{2} & -\frac{z-z^{-1}}{2 i} \\
\frac{z-z^{-1}}{2 i} & \frac{z+z^{-1}}{2}
\end{array}\right), \quad \text { para todo } z \in \mathbb{C} \backslash\{0\}
$$

e

$$
\mathscr{R}(z)=\left(\begin{array}{cc}
\frac{z^{2}+1}{2} & -\frac{z^{2}-1}{2 i} \\
\frac{z^{2}-1}{2 i} & \frac{z^{2}+1}{2}
\end{array}\right)=z S_{z} \quad \text { para todo } z \in \mathbb{C} .
$$

Em que nesta ocasião tomamos $n \in \mathbb{N}$ e $A_{1}, \ldots, A_{n}$ matrizes em $\operatorname{SL}(2, \mathbb{R})$, para então definirmos, para $z \in \mathbb{C}$, a matriz

$$
C_{z}:=\prod_{j=1}^{n} A_{j} \mathscr{R}(z)=A_{n} \mathscr{R}(z) \ldots A_{1} \mathscr{R}(z) .
$$

O principal resultado técnico é a seguinte proposição : 
Proposição 4.1 (Parametrização holomorfa do espectro de $C_{z}$ )

Existem funções holomorfas $\lambda_{1}, \lambda_{2}: \mathbb{D} \rightarrow \mathbb{C}$, tais que para todo $z \in \mathbb{D}$ $\left\{\lambda_{1}(z), \lambda_{2}(z)\right\}$ é o espectro de $C_{z}$ e temos $\left|\lambda_{2}(z)\right|<\left|\lambda_{1}(z)\right|$.

Isso mostra que a função $z \mapsto \log \left|\lambda_{1}(z)\right|=\log \rho\left(C_{z}\right)$ é harmônica em $\mathbb{D}$, além disso, mostraremos que esta função é contínua em $\overline{\mathbb{D}}$, portanto, ela irá satisfazer a propriedade do valor médio para funções harmônicas o que implicará em obtermos a igualdade para o Teorema da Desigualdade de Herman. Como veremos, a prova deste resultado utilizará um compilado de resultados técnicos que serão apresentados em detalhes.

Deve-se mencionar, nos resultados que seguem, exceto quando indicarmos o contrário, estaremos considerando por $\|\cdot\|$ a norma do operador, ou seja, dada uma matriz complexa ou real denotamos $\|A\|=\sup _{v \neq 0} \frac{|A v|}{|v|}$, sendo $|\cdot|$ a norma euclidiana do espaço em que $v$ faça sentido, ou ainda o valor absoluto para os reais. Em grande parte do texto estaremos interessados em provar resultados sobre o espaço $\mathrm{SL}(2, \mathbb{R})$, sendo assim começaremos provando um resultado simples, porém bastante utilizado, à respeito da norma do operador restrita a este espaço, contudo, precisamos da Desigualdade de Hadamard, a qual será enunciada a seguir.

Teorema 4.2 (Desigualdade de Hadamard) Seja $A \in \operatorname{Mat}(n, \mathbb{C})$ uma matriz com colunas $v_{1}, \ldots, v_{n}$, então

$$
|\operatorname{det} A| \leq \prod_{i=1}^{n}\left|v_{i}\right|
$$

Corolário 4.3 Se $A \in S L(2, \mathbb{R})$, então $\|A\| \geq 1$.

Prova. Seja $A \in \mathrm{SL}(2, \mathbb{R}), e_{1}=(1,0), e_{2}=(0,1)$ e $|\cdot|$ a norma euclidiana em $\mathbb{R}^{2}$. Pela definição da norma do operador, para todo $v \in \mathbb{R}^{2}$ temos $|A v| \leq \| A|||v|$, em particular $\left|A e_{1}\right| \leq\|A\|$ e $\left|A e_{2}\right| \leq\|A\|$. Considere por $v_{j}$ a $j$-ésima coluna de $A$, então $v_{j}=A e_{j}$ para $j \in\{1,2\}$, portanto $\|A\|^{2} \geq\left|A e_{1}\right|\left|A e_{2}\right|=\left|v_{1}\right|\left|v_{2}\right|$, assim pela Desigualdade de Hadamard temos $1=|\operatorname{det} A| \leq\left|v_{1}\right|\left|v_{2}\right| \leq\|A\|^{2}$, como $\|A\| \geq 0$, temos $\|A\| \geq 1$.

Definiremos para os resultados que se seguem ao longo do capítulo a seguinte matriz especial em $\mathrm{SL}(2, \mathbb{R})$

$$
H_{c}=\left(\begin{array}{cc}
c & 0 \\
0 & c^{-1}
\end{array}\right),
$$


em que assumimos $c \geq 1$. Antes de prosseguirmos darei breve atenção a uma consequência, talvez não tão rotineira, de um fato conhecido, haja vista que este argumento será utilizado em outras etapas no decorrer do capítulo. O Teorema da Decomposição Polar, fato bastante conhecido, nos fornece uma extensão conhecida como o Teorema da Decomposição em Valores Singulares, o qual permite que uma matriz $A$ de dimensão $m \times n$, real ou complexa, obtenha uma fatorização da forma $A=U \Sigma V^{*}$, em que:

Observação 4.4 No caso em que $A$ é uma matriz real $n \times n$, o que será nosso caso, as matrizes $U$ e $V^{*}$ podem ser tomadas de modo a serem reais de dimensão $n \times n$, e neste caso serem unitárias equivale a serem ortonormais. Ainda mais, $U$ e $V^{*}$ representam rotações ou reflexões no espaço em questão, enquanto $\Sigma$ é uma matriz diagonal $n \times n$.

Observação 4.5 Em particular, no caso em que $\operatorname{det} A$ é positivo, ambas as matrizes $U$ e $V^{*}$ podem ser tomadas de modo a serem ambas rotações, ou reflexões.

Portanto, pelas Observações acima, e pelo fato de tomarmos sempre $A \in \mathrm{SL}(2, \mathbb{R})$, podemos representar $U$ e $V^{*}$ como matrizes de rotação, ou seja, existem $\alpha, \beta \in[0,2 \pi]$ tais que $U=R_{\beta}$ e $V^{*}=R_{\alpha}$, e ainda como $1=\operatorname{det} A=\operatorname{det} \Sigma$, consequentemente $\Sigma$ deve ser uma matriz da forma $\Sigma_{1}=\left(\begin{array}{cc}c & 0 \\ 0 & c^{-1}\end{array}\right)$, ou $\Sigma_{2}=\left(\begin{array}{cc}c^{-1} & 0 \\ 0 & c\end{array}\right)$ com $c>0$. Contudo, note que $R_{\frac{3 \pi}{2}} \Sigma_{2} R_{\frac{\pi}{2}}=\Sigma_{1}$. Então, sem perda de generalidade, podemos assumir $c \geq 1 \mathrm{e}$ considerar $\Sigma=\left(\begin{array}{cc}c & 0 \\ 0 & c^{-1}\end{array}\right)=H_{c}$, de modo a obtermos $\alpha, \beta \in[0,2 \pi]$ e $c \geq 1$ tais que $A=R_{\beta} H_{c} R_{\alpha}$, já que para quaisquer $\alpha$ e $\beta$ tomados acima, existirão $\alpha^{\prime}$ e $\beta^{\prime}$ tais que $A=R_{\beta} H_{c} R_{\alpha}=R_{\beta^{\prime}} R_{\beta} H_{c} R_{\alpha} R_{\alpha^{\prime}}$, em que $R_{\beta^{\prime}} R_{\beta}$ e $R_{\alpha} R_{\alpha^{\prime}}$ são ainda rotações.

Observação 4.6 Note ainda que $\left\|H_{c}\right\|=\sup _{\left|v=\left(v_{1}, v_{2}\right)\right|=1}\left|H_{c} v\right|=$ $\sup _{\left|v=\left(v_{1}, v_{2}\right)\right|=1} \sqrt{c^{2} v_{1}^{2}+c^{-2} v_{2}^{2}}=\sqrt{c^{2}}=c$, já que $c>0$, e com isso $\|A\|=\left\|R_{\beta} H_{c} R_{\alpha}\right\|=\left\|R_{\beta} H_{c}\right\|=\left\|H_{c}\right\|=c$.

Pois bem, começaremos, efetivamente, o capítulo, definindo uma quantidade para as matrizes $A \in \mathrm{SL}(2, \mathbb{R})$ dada por

$$
N(A)=\log \left(\frac{\|A\|+\|A\|^{-1}}{2}\right) .
$$

Seguidamente, veremos uma proposição a qual tem por objetivo nos dar uma interpretação da quantidade $N(A)$ definida acima. Assim, sendo possível 
perceber que para uma matriz $A \in \mathrm{SL}(2, \mathbb{R})$, o número $N(A)$ pode ser visto como a "taxa média exponencial de expansão" da matrix $A$.

Proposição 4.7 Seja $A \in S L(2, \mathbb{R})$. Então

$$
N(A)=\frac{1}{2 \pi} \int_{0}^{2 \pi} \log \|A(\cos \theta, \sin \theta)\| d \theta
$$

Prova. Como já discutido no texto deste capítulo, e evidenciado em particular pelas Observações 4.4, 4.5 e 4.6, o Teorema da Decomposição Polar, na realidade uma consequência deste, o Teorema da Decomposição em Valores Singulares, nos permite afirmar a existência de números $\alpha, \beta \in[0,2 \pi]$ e $c \geq 1$ tais que $A=R_{\beta} H_{c} R_{\alpha}$. Como já calculado lembre-se que $\|A\|=\left\|H_{c}\right\|=c$, além disso perceba que para $(\cos \theta, \sin \theta) \in S^{1} \subset \mathbb{R}^{2}$ temos

$$
\begin{aligned}
\|A(\cos \theta, \sin \theta)\| & =\left\|R_{\beta} H_{c} R_{\alpha}(\cos \theta, \sin \theta)\right\|=\left\|H_{c}\left(R_{\alpha}(\cos \theta, \sin \theta)\right)\right\| \\
& =\left\|H_{c}(\cos (\alpha+\theta), \sin (\alpha+\theta))\right\|,
\end{aligned}
$$

assim podemos notar que ao calcularmos a taxa média referida, não fará diferença considerar $A$ ou $H_{c}$. Então, sem perda de generalidade podemos supor $A=H_{c}$ e portanto temos

$$
\begin{aligned}
\int_{0}^{2 \pi} \log \|A(\cos \theta, \sin \theta)\| d \theta & =\int_{0}^{2 \pi} \log \left\|H_{c}(\cos \theta, \sin \theta)\right\| d \theta \\
& =\int_{0}^{2 \pi} \log \left(c^{2} \cos ^{2} \theta+c^{-2} \sin ^{2} \theta\right)^{\frac{1}{2}} d \theta \\
& =\frac{1}{2} \int_{0}^{2 \pi} \log \left(c^{2} \cos ^{2} \theta+c^{-2} \sin ^{2} \theta\right) d \theta \\
& =\int_{0}^{\pi} \log \left(c^{2} \cos ^{2} \theta+c^{-2} \sin ^{2} \theta\right) d \theta \\
& =\int_{0}^{\pi} \log \left(\frac{\left(c^{2}\right)^{2} \cos ^{2} \theta+\sin ^{2} \theta}{c^{2}}\right) d \theta \\
& =\int_{0}^{\pi} \log \left(\left(c^{2}\right)^{2} \cos ^{2} \theta+\sin ^{2} \theta\right) d \theta-\int_{0}^{\pi} \log c^{2} d \theta \\
& =\int_{0}^{\pi} \log \left(\left(c^{2}\right)^{2} \cos ^{2} \theta+\sin ^{2} \theta\right) d \theta-2 \pi \log c .
\end{aligned}
$$

Defina as funções

$$
\begin{array}{rlrl}
f: \mathbb{R} \times(0, \pi) & \longrightarrow \mathbb{R} & F: \mathbb{R} & \longrightarrow \mathbb{R} \\
(b, \theta) & \longmapsto \log \left(b^{2} \cos ^{2} \theta+\sin ^{2} \theta\right) & b & \longmapsto \int_{0}^{\pi} f(b, \theta) d \theta,
\end{array}
$$

então

$$
\int_{0}^{2 \pi} \log \|A(\cos \theta, \sin \theta)\| d \theta=F\left(c^{2}\right)-2 \pi \log c .
$$


Portanto o objetivo será avaliar a função $F$.

Afirmamos que $F^{\prime}(b)=2 b \int_{0}^{\pi} \frac{1}{b^{2}+\tan ^{2} \theta} d \theta$, de fato, pelo modo como definimos as funções $f$ e $F$ temos

$$
\begin{aligned}
\frac{\partial f}{\partial b}: \mathbb{R} \times(0, \pi) & \longrightarrow \mathbb{R} \\
(b, \theta) & \longmapsto \frac{2 b}{b^{2}+\tan ^{2} \theta}=\frac{2 b \cos ^{2} \theta}{b^{2} \cos ^{2} \theta+\sin ^{2} \theta},
\end{aligned}
$$

e note que, $b^{2} \cos ^{2} \theta+\sin ^{2} \theta \neq 0$ em $\mathbb{R} \times(0, \pi)$, então $f$ e $\frac{\partial f}{\partial b}$ estão bem definidas e são claramente contínuas em $\mathbb{R} \times(0, \pi)$. Considere a sequência $\left(\left[b_{0}, b_{1}\right] \times\left[\theta_{0_{n}}, \theta_{\pi_{n}}\right]\right)_{n \in \mathbb{N}}$ em que $\left[b_{0}, b_{1}\right] \subset \mathbb{R}$ e $\left[\theta_{0_{n}}, \theta_{\pi_{n}}\right] \subset(0, \pi)$ para todo $n \in \mathbb{N}, \operatorname{com}_{\lim _{n \rightarrow \infty}}\left(\theta_{0_{n}}, \theta_{\pi_{n}}\right)=(0, \pi)$. Para cada $n \in \mathbb{N}$ defina a função

$$
\begin{aligned}
F_{n}: \mathbb{R} & \longrightarrow \mathbb{R} \\
b & \longmapsto \int_{\theta_{0_{n}}}^{\theta_{\pi_{n}}} f(b, \theta) d \theta,
\end{aligned}
$$

perceba que $f$ e $\frac{\partial f}{\partial b}$ são em particular contínuas em $\left[b_{0}, b_{1}\right] \times\left[\theta_{0_{n}}, \theta_{\pi_{n}}\right]$, portanto a Regra de Leibniz é aplicável e assim temos $F_{n}^{\prime}(b)=\int_{\theta_{0_{n}}}^{\theta_{\pi_{n}}} \frac{\partial f}{\partial b}(b, \theta) d \theta$, além disto,

$$
\lim _{n \rightarrow \infty} F_{n}(b)=\lim _{n \rightarrow \infty} \int_{\theta_{0_{n}}}^{\theta_{\pi_{n}}} f(b, \theta) d \theta=\int_{0}^{\pi} f(b, \theta) d \theta=F(b)
$$

e

$$
\lim _{n \rightarrow \infty} F_{n}^{\prime}(b)=\lim _{n \rightarrow \infty} \int_{\theta_{0_{n}}}^{\theta_{\pi_{n}}} \frac{\partial f}{\partial b}(b, \theta) d \theta=\int_{0}^{\pi} \frac{\partial f}{\partial b}(b, \theta) d \theta
$$

no compacto $\left[b_{0}, b_{1}\right]$, deste modo, $\left(F_{n}^{\prime}\right)_{n \in \mathbb{N}}$ converge uniformemente para $\int_{0}^{\pi} \frac{\partial f}{\partial b}(b, \theta) d \theta$, então sabemos que "o limite da derivada é igual a derivada do limite", portanto $\lim _{n \rightarrow \infty} F_{n}^{\prime}(b)=F^{\prime}(b)$. Agora, tomando $x=\tan \theta, \arctan x=\theta$ e $d x=\sec ^{2} \theta d \theta$ obtemos

$$
\begin{aligned}
F^{\prime}(b)=2 b \int_{0}^{\pi} \frac{1}{b^{2}+\tan ^{2} \theta} d \theta & =2 b \int_{-\infty}^{+\infty} \frac{1}{b^{2}+x^{2}} \frac{1}{\sec ^{2}(\arctan x)} d x \\
& =2 b \int_{-\infty}^{+\infty} \frac{1}{\left(b^{2}+x^{2}\right)\left(x^{2}+1\right)} d x
\end{aligned}
$$

então precisamos calcular a integral acima, para tal utilizaremos o Teorema dos Resíduos, Teorema 3.33. Seja

$$
R(z)=\frac{1}{\left(b^{2}+z^{2}\right)\left(z^{2}+1\right)}=\frac{1}{(z-b i)(z+b i)(z-i)(z+i)},
$$

$p(z)=\left(b^{2}+z^{2}\right)\left(z^{2}+1\right)$ e $S=\{-i, i,-b i, b i\}$ o conjunto das raízes de $p$. É fácil 
perceber que as singularidades de $R$ são justamente as raízes do polinômio $p$, pois $R$ é holomorfa em $\mathbb{C} \backslash S$. Já que $R$ não tem singularidades na reta real, faz sentido considerarmos a curva $C_{r}=[-r, r] * \gamma_{r}$, dada pela concatenação dos caminhos $[-r, r]$ e $\gamma_{r}$, que representam o segmento de reta que vai de $(-r, 0)$ à $(r, 0)$, nesse sentido, e o semicírculo que vai de $(r, 0)$ à $(-r, 0)$, neste sentido anti-horário, respectivamente, ou seja, temos $[-r, r](t)=t r-(1-t) r$, $t \in[0,1]$ e $\gamma_{r}(\theta)=r e^{i \theta}, \theta \in[0, \pi]$, e então $C_{r}$ pode ser representada como na figura abaixo

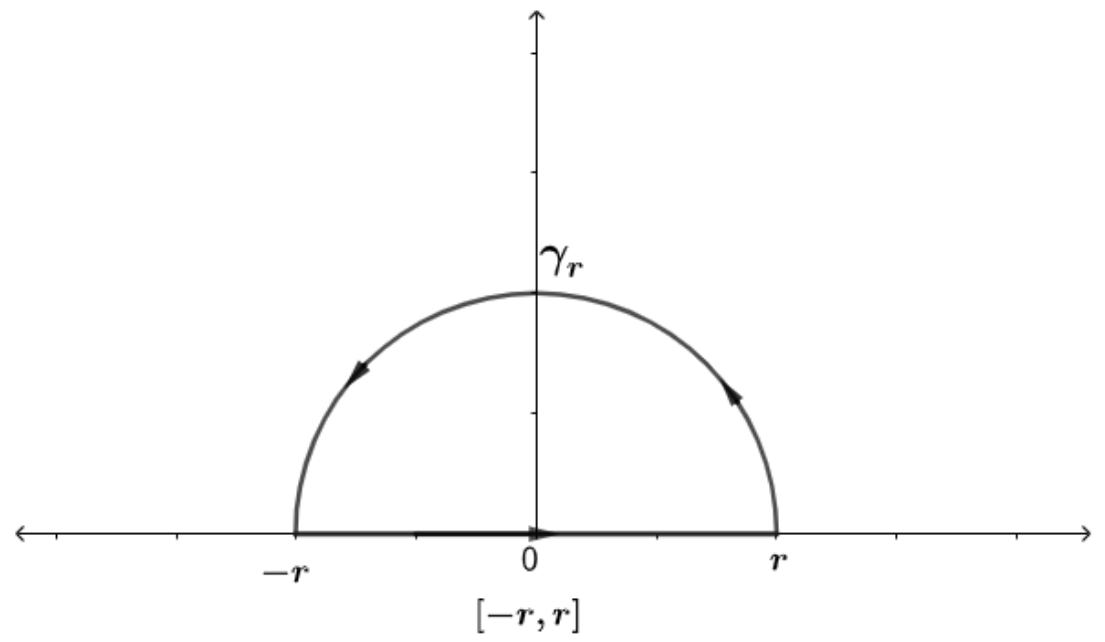

Figura 4.1: Caminho $C_{r}=[-r, r] * \gamma_{r}$

Sabemos que $\mathbb{C}$ é um conjunto estrelado e $C_{r} \subset \mathbb{C} \backslash S$ para todo $r$ suficientemente grande. Portanto o Teorema dos Resíduos é aplicável e assim

$$
\int_{C_{r}} R(z) d z=2 \pi i \sum_{a \in S} \operatorname{Res}(R, a) \operatorname{ind}_{C_{r}}(a)
$$

Como $C_{r}=[-r, r] * \gamma_{r}$, temos por definição que

$$
\int_{C_{r}} R(z) d z=\int_{[-r, r]} R(z) d z+\int_{\gamma_{r}} R(z) d z=\int_{-r}^{r} R(z) d z+\int_{\gamma_{r}} R(z) d z
$$

Pela Proposição 3.19, segue

$$
\begin{aligned}
\left|\int_{\gamma_{r}} R(z) d z\right| & \leq l\left(\gamma_{r}\right) \sup _{z \in \gamma_{r}}|R(z)|=\pi r \sup _{|z|=r} \frac{1}{\left|\left(b^{2}+z^{2}\right)\left(z^{2}+1\right)\right|} \\
& =\pi \sup _{|z|=r} \frac{r}{\left|\left(b^{2}+z^{2}\right)\left(z^{2}+1\right)\right|}=\pi \sup _{|z|=r}\left|\frac{1}{b^{2}+z^{2}}\right|\left|\frac{z}{z^{2}+1}\right| \\
& =\pi \sup _{|z|=r}\left|\frac{1}{b^{2}+z^{2}}\right|\left|\frac{1}{\frac{1}{z}+z}\right| \longrightarrow 0, \text { quando } r \longrightarrow \infty
\end{aligned}
$$

Portanto, claramente $\int_{\gamma_{r}} R(z) d z \rightarrow 0$, quando $r \rightarrow \infty$, perceba ainda que 
considerando $\mathbb{H}=\{c+i d ; c \in \mathbb{R}, d>0\}$, o semiplano superior, temos

$$
\lim _{r \rightarrow \infty} \int_{C_{r}} R(z) d z=\lim _{r \rightarrow \infty} 2 \pi i \sum_{a \in S} \operatorname{Res}(R, a) \operatorname{ind}_{C_{r}}(a)=2 \pi i \sum_{a \in S \cap \mathbb{H}} \operatorname{Res}(R, a),
$$

já que para todo $a \notin \mathbb{H}$ e para todo $r \geq 0$ temos $\operatorname{ind}_{C_{r}}(a)=0$, e para todo $a \in \mathbb{H}$, $\operatorname{ind}_{C_{r}}(a)=1$, quando $r \rightarrow \infty$. Deste modo, como

$$
\lim _{r \rightarrow \infty} \int_{-r}^{r} R(z) d z=\int_{-\infty}^{\infty} \frac{1}{\left(z^{2}+1\right)\left(b^{2}+1\right)} d z
$$

então por 4-2 e 4-3 segue

$$
\int_{-\infty}^{\infty} \frac{1}{\left(z^{2}+1\right)\left(b^{2}+1\right)} d z=2 \pi i \sum_{a \in S \cap \mathbb{H}} \operatorname{Res}(R, a)
$$

Como não sabemos se $b>0$ ou $b<0$, não podemos afirmar se $b i \in \mathbb{H}$ ou $-b i \in \mathbb{H}$, assim teremos que calcular os resíduos de $R$ nos pontos $-b i$, bi e $i$. Sabemos que todas as singularidades de $R$ são pólos, em particular de ordem 1 , dessa maneira, pela Observação 3.32 podemos calcular os resíduos em questão da seguinte maneira :

- $z=i$

$$
\operatorname{Res}(R, i)=\lim _{z \rightarrow i}(z-i) R(z)=\lim _{z \rightarrow i} \frac{1}{\left(b^{2}+z^{2}\right)(z+i)}=\frac{1}{2 i\left(b^{2}-1\right)}
$$

- $z=b i$

$$
\operatorname{Res}(R, b i)=\lim _{z \rightarrow b i}(z-b i) R(z)=\lim _{z \rightarrow b i} \frac{1}{\left(z^{2}+1\right)(z+b i)}=-\frac{1}{2 i b\left(b^{2}-1\right)}
$$

- $z=-b i$

$$
\operatorname{Res}(R,-b i)=\lim _{z \rightarrow-b i}(z+b i) R(z)=\lim _{z \rightarrow-b i} \frac{1}{\left(z^{2}+1\right)(z-b i)}=\frac{1}{2 i b\left(b^{2}-1\right)}
$$

Podemos então perceber que o valor $\operatorname{Res}(R, i)+\operatorname{Res}(R, b i)$ quando $b>0$, é o mesmo que $\operatorname{Res}(R, i)+\operatorname{Res}(R,-b i)$ quando $b<0$, ou seja, faz sentido assumirmos tanto $b>0$, quanto $b<0$, que o resultado não se alterará. Vale observar que pelo modo como definimos $F^{\prime}$, esta se anula quando $b=0$, então não precisamos nos preocupar quando $b=0$. Sem perda de generalidade, podemos considerar $b>0$ para o restante da prova, dessa maneira $i, b i \in \mathbb{H}$ e portanto

$$
\int_{-\infty}^{\infty} \frac{1}{\left(b^{2}+z^{2}\right)\left(z^{2}+1\right)} d z=2 \pi i(\operatorname{Res}(R, i)+\operatorname{Res}(R, b i))=\frac{\pi}{b(b+1)}
$$


Voltando para a variável $x$ temos $2 b \int_{-\infty}^{\infty} \frac{1}{\left(b^{2}+x^{2}\right)\left(x^{2}+1\right)}=\frac{2 \pi}{b+1}$. Então, $F^{\prime}(b)=\frac{2 \pi}{b+1}$ e ainda $F(1)=\int_{0}^{\pi} \log \left(\cos ^{2} \theta+\sin ^{2} \theta\right) d \theta=(\log 1)(\pi-0)=0$. Portanto, temos a equação diferencial $F^{\prime}(b)=\frac{2 \pi}{b+1}$, com condição inicial $F(1)=0$. Solucionando a equação:

$$
F^{\prime}(b)=\frac{2 \pi}{b+1} \Rightarrow F(b)=2 \pi \int \frac{1}{b+1} d b=2 \pi \log (b+1)+C,
$$

sendo $C$ a constante de integração. Assim

$$
\begin{gathered}
0=F(1)=2 \pi \log 2+C \Rightarrow C=-2 \pi \log 2=2 \pi \log \frac{1}{2} \\
\Rightarrow F(b)=2 \pi \log (b+1)+2 \pi \log \frac{1}{2}=2 \pi \log \frac{1}{2}(b+1) .
\end{gathered}
$$

Assim, $F\left(c^{2}\right)=2 \pi \log \frac{1}{2}\left(c^{2}+1\right)$ e consequentemente por $4-1$ temos

$$
\begin{aligned}
\int_{0}^{2 \pi} \log \|A(\cos \theta, \sin \theta)\| d \theta & =F\left(c^{2}\right)-2 \pi \log c=2 \pi \log \frac{1}{2}\left(c^{2}+1\right)-2 \pi \log c \\
& =2 \pi \log \left(\frac{c^{2}+1}{2 c}\right)=2 \pi \log \frac{1}{2}\left(\|A\|+\|A\|^{-1}\right) \\
& =2 \pi N(A) .
\end{aligned}
$$

Antes de tratarmos do próximo resultado que possui relevância neste capítulo, precisaremos elucidar a continuidade da função raio espectral, $\rho$, a qual está definida para matrizes, de um modo geral, e trata-se do valor máximo absoluto entre os autovalores da matriz avaliada.

Proposição 4.8 (Continuidade do Raio Espectral) O raio espectral

$$
\begin{aligned}
\rho: \operatorname{Mat}(2, \mathbb{C}) & \longrightarrow[0,+\infty) \\
A & \longmapsto \max \left\{\left|\lambda_{1}(A)\right|,\left|\lambda_{2}(A)\right|\right\}
\end{aligned}
$$

é uma função contínua, em que $\lambda_{1}(A)$ e $\lambda_{2}(A)$ são os autovalores de $A$.

Prova. Seja $\mathcal{P}_{2}(\mathbb{C})$ o conjunto dos polinômios de grau 2 com coeficientes complexos. Considere por $p_{A}$ o polinômio característico de $A$, isto é, $p_{A}(z)=z^{2}-z \operatorname{tr} A+\operatorname{det} A$, e ainda $\lambda_{1}(p), \lambda_{2}(p)$ as raízes de um polinômio $p \in \mathcal{P}_{2}(\mathbb{C})$, então $\rho$ pode ser escrita como a composição das funções a seguir 


$$
\begin{array}{rlrl}
\psi: \operatorname{Mat}(2, \mathbb{C}) & \longrightarrow \mathcal{P}_{2}(\mathbb{C}) \quad \text { e } \quad \varphi: \mathcal{P}_{2}(\mathbb{C}) & \longrightarrow[0,+\infty) \\
A & \longmapsto p_{A} & p & \longmapsto \max \left\{\left|\lambda_{1}(p)\right|,\left|\lambda_{2}(p)\right|\right\}
\end{array}
$$

Deste modo, é suficiente mostrarmos que $\psi$ e $\varphi$ são funções contínuas, começaremos pela função $\psi$. Estaremos considerando os espaços $(\mathbb{C},|\cdot|)$, $(\operatorname{Mat}(2, \mathbb{C}),\|\cdot\|),\left(\mathcal{P}_{2}(\mathbb{C}),\|\cdot\|_{1}\right)$, em que $|\cdot|$ é a norma euclidiana em $\mathbb{C}$, $\|\cdot\|$ é a norma do operador e $\|\cdot\|_{1}$ é a norma usual sobre os polinômios, isto é, para um polinômio $p(z)=\sum_{i=0}^{2} a_{i} z^{i}$ temos $\|p(z)\|_{1}=\sum_{i=0}^{2}\left|a_{i}\right|$. Seja $A \in \operatorname{Mat}(2, \mathbb{C})$, como as funções determinante e traço são funções contínuas, sabemos que para quaisquer $\epsilon_{1}, \epsilon_{2}>0$, existem $\delta_{1}, \delta_{2}>0$ em que para todo $B \in \operatorname{Mat}(2, \mathbb{C})$ tal que $\|A-B\|<\delta_{1}$ temos $|\operatorname{det} A-\operatorname{det} B|<\epsilon_{1}$ e tal que $\|A-B\|<\delta_{2}$ temos $|\operatorname{tr} A-\operatorname{tr} B|<\epsilon_{2}$. Tome $\epsilon=2 \max \left\{\epsilon_{1}, \epsilon_{2}\right\}, \delta=\min \left\{\delta_{1}, \delta_{2}\right\}$, e assim para todo $B \in \operatorname{Mat}(2, \mathbb{C})$ tal que $\|A-B\|<\delta$, tem-se

$$
\begin{aligned}
\|\psi(A)-\psi(B)\|_{1} & =\|z(\operatorname{tr} B-\operatorname{tr} A)+\operatorname{det} A-\operatorname{det} B\|_{1} \\
& =|\operatorname{tr} B-\operatorname{tr} A|+|\operatorname{det} A-\operatorname{det} B|<\epsilon_{1}+\epsilon_{2}<\epsilon .
\end{aligned}
$$

O que prova a continuidade de $\psi$. Agora, provaremos a continuidade de $\varphi$. Considere o polinômio $p(z)=c_{0}+c_{1} z+c_{2} z^{2} \in \mathcal{P}_{2}(\mathbb{C})$ com raizes $\lambda_{1}(p)$ e $\lambda_{2}(p)$, e fixemos $\epsilon>0$. Seja $g \in \mathcal{P}_{2}(\mathbb{C})$ um polinômio suficientemente próximo de $p$, isto é, tal que $d(z):=g(z)-p(z)$ tem coeficientes suficientemente pequenos. Temos duas possibilidades, $\lambda_{1}(p) \neq \lambda_{2}(p)$ e $\lambda_{1}(p)=\lambda_{2}(p)$.

(i) $\lambda_{1}(p) \neq \lambda_{2}(p)$.

Sem perda de generalidade estamos tomando $\epsilon$ de modo que, para todo $z \in \partial B\left(\lambda_{i}(p), \epsilon\right)$ tenhamos $p(z) \neq 0$, para $i \in\{1,2\}$, e tal que $\bar{B}\left(\lambda_{1}(p), \epsilon\right) \cap \bar{B}\left(\lambda_{2}(p), \epsilon\right)=\emptyset$. Seja $d(z)=d_{0}+d_{1} z+d_{2} z^{2} \in \mathcal{P}_{2}(\mathbb{C})$, para todo $z \in B\left(\lambda_{i}(p), \epsilon\right) \subset \mathbb{C}$ temos

$$
\begin{aligned}
|d(z)| & \leq\left|d_{0}\right|+\left|d_{1}\right||z|+\left|d_{2}\right||z|^{2} \\
& \leq\left|d_{0}\right|+\left|d_{1}\right|\left(\left|\lambda_{i}(p)\right|+\epsilon\right)+\left|d_{2}\right|\left(\left|\lambda_{i}(p)\right|+\epsilon\right)^{2}
\end{aligned}
$$

para $i \in\{1,2\}$. Como $\partial B\left(\lambda_{i}(p), \epsilon\right)$ é compacto e para todo $z \in \partial B\left(\lambda_{i}(p), \epsilon\right)$ temos $p(z) \neq 0$, em particular temos $0<\inf _{z \in \partial B\left(\lambda_{i}(p), \epsilon\right)}|p(z)|$. Assim, podemos tomar os coeficientes do polinômio $d$, isto é, $d_{0}, d_{1}$ e $d_{2}$, com norma suficientemente pequena, de 
modo que para todo $z \in \partial B\left(\lambda_{i}(p), \epsilon\right)$ tenhamos

$$
\begin{aligned}
|d(z)| & \leq\left|d_{0}\right|+\left|d_{1}\right|\left(\left|\lambda_{i}(p)\right|+\epsilon\right)+\left|d_{2}\right|\left(\left|\lambda_{i}(p)\right|+\epsilon\right)^{2} \\
& <\inf _{z \in \partial B\left(\lambda_{i}(p), \epsilon\right)}|p(z)| \leq|p(z)|
\end{aligned}
$$

para $i \in\{1,2\}$. Portanto, pelo Teorema de Rouché, Teorema 3.35, os polinômios $p$ e $p+d$ possuem a mesma quantidade de raizes em $B\left(\lambda_{i}(p), \epsilon\right)$ para $i \in\{1,2\}$, ou seja, o polinômio $g=p+d$ tem raizes $\lambda_{1}(g) \in B\left(\lambda_{1}(p), \epsilon\right)$ e $\lambda_{2}(g) \in B\left(\lambda_{2}(p), \epsilon\right)$. As raizes do polinômio $p$ fixado inicialmente, ainda nos fornecem duas possibilidades, $\left|\lambda_{1}(p)\right| \neq\left|\lambda_{2}(p)\right| \mathrm{e}$ $\left|\lambda_{1}(p)\right|=\left|\lambda_{2}(p)\right|$.

(a) $\left|\lambda_{1}(p)\right| \neq\left|\lambda_{2}(p)\right|$.

Sem perda de generalidade, assuma $\left|\lambda_{1}(p)\right|>\left|\lambda_{2}(p)\right|$. Note que estamos exigindo de $\epsilon$ apenas $\bar{B}\left(\lambda_{1}(p), \epsilon\right) \cap \bar{B}\left(\lambda_{2}(p), \epsilon\right)=\emptyset \mathrm{e}$ $p(z) \neq 0$ para todo $z \in \partial B\left(\lambda_{i}(p), \epsilon\right)$ com $i \in\{1,2\}$. Portanto, sua arbitrariedade nos permite tomar $\epsilon$ suficientemente pequeno, de moto que $\left|\lambda_{1}(p)\right|>\left|\lambda_{2}(p)\right|$ implique em termos $\left|\lambda_{1}(g)\right|>\left|\lambda_{2}(g)\right|$, consequentemente

$$
\begin{aligned}
|\varphi(p)-\varphi(g)| & =\left|\max \left\{\left|\lambda_{1}(p)\right|,\left|\lambda_{2}(p)\right|\right\}-\max \left\{\left|\lambda_{1}(g)\right|,\left|\lambda_{2}(g)\right|\right\}\right| \\
& =|| \lambda_{1}(p)|-| \lambda_{1}(g)|| \leq\left|\lambda_{1}(p)-\lambda_{1}(g)\right|<\epsilon,
\end{aligned}
$$

pois $\lambda_{1}(g) \in B\left(\lambda_{1}(p), \epsilon\right)$. O caso em que $\left|\lambda_{1}(p)\right|<\left|\lambda_{2}(p)\right|$ se dá de forma análoga.

(b) $\left|\lambda_{1}(p)\right|=\left|\lambda_{2}(p)\right|$.

Suponha que $\left|\lambda_{1}(g)\right| \geq\left|\lambda_{2}(g)\right|$, então

$$
\begin{aligned}
|\varphi(p)-\varphi(g)| & =\left|\max \left\{\left|\lambda_{1}(p)\right|,\left|\lambda_{2}(p)\right|\right\}-\max \left\{\left|\lambda_{1}(g)\right|,\left|\lambda_{2}(g)\right|\right\}\right| \\
& =|| \lambda_{1}(p)|-| \lambda_{1}(g)|| \leq\left|\lambda_{1}(p)-\lambda_{1}(g)\right|<\epsilon .
\end{aligned}
$$

Análogo para o caso em que $\left|\lambda_{1}(g)\right|<\left|\lambda_{2}(g)\right|$.

(ii) $\lambda_{1}(p)=\lambda_{2}(p)$

Temos $\left|\lambda_{1}(p)\right|=\left|\lambda_{2}(p)\right|$ e a prova se dá de forma idêntica a apresentada anteriormente, exceto pelo fato de não exigirmos $\bar{B}\left(\lambda_{1}(p), \epsilon\right) \cap \bar{B}\left(\lambda_{2}(p), \epsilon\right)=\emptyset$, o que não faria sentido, pois neste caso $\bar{B}\left(\lambda_{1}(p), \epsilon\right)=\bar{B}\left(\lambda_{2}(p), \epsilon\right)$ e, de fato, a prova continua a valer, já que o Teorema de Rouché nos fornece que o polinômio $g=p+d$ possui a 
mesma quantidade de zeros em $B\left(\lambda_{1}(p), \epsilon\right)=B\left(\lambda_{2}(p), \epsilon\right)$, contados com sua multiplicidade, ou seja, $g$ possuirá duas raízes na bola acima.

O próximo resultado a ser estudado se dedica em provar a continuidade de uma função definida com respeito à integral de Riemann e o raio espectral. É relevante mencionar que em todo o restante do capítulo estaremos considerando para $\theta \in \mathbb{R}, R_{\theta}=\left(\begin{array}{cc}\cos \theta & -\sin \theta \\ \sin \theta & \cos \theta\end{array}\right)$, isto é, $R_{\theta}$ representa a matriz de rotação por um ângulo $\theta$.

Lema 4.9 Seja $n \in \mathbb{N}$, a função

$$
\begin{aligned}
f: S L(2, \mathbb{R})^{n} \times[0,2 \pi] & \longrightarrow \mathbb{R} \\
(A, \theta)=\left(A_{1}, \ldots, A_{n}, \theta\right) & \longmapsto \int_{0}^{2 \pi} \log \rho\left(A_{n} R_{\theta} \ldots A_{1} R_{\theta}\right) d \theta
\end{aligned}
$$

é contínua.

Prova. Sabemos que as funções $\log :(0+\infty) \rightarrow \mathbb{R}$ e $\Theta: \mathbb{R} \rightarrow \mathrm{SO}(2, \mathbb{R})$, em que $\Theta(\theta)=R_{\theta}=\left(\begin{array}{cc}\cos \theta & -\sin \theta \\ \sin \theta & \cos \theta\end{array}\right)$ são contínuas, além disso, na proposição anterior, Proposição 4.8, provamos que $\rho: \operatorname{Mat}(2, \mathbb{C}) \rightarrow[0,+\infty)$ é uma função contínua, consequentemente $\rho \uparrow_{\mathrm{SL}(2, \mathbb{R})}: \mathrm{SL}(2, \mathbb{R}) \rightarrow[0,+\infty)$ é uma função contínua e portanto a função definida por

$$
\begin{aligned}
\tilde{f}: \operatorname{SL}(2, \mathbb{R})^{n} \times \mathbb{R} & \longrightarrow \mathbb{R} \\
(A, \theta)=\left(A_{1}, \ldots, A_{n}, \theta\right) & \longmapsto \log \rho\left(A_{n} R_{\theta} \ldots A_{1} R_{\theta}\right)
\end{aligned}
$$

é também uma função contínua, já que para qualquer sequência $\left(A_{j}, \theta_{j}\right)=\left(A_{j_{1}}, \ldots, A_{j_{n}}, \theta_{j}\right) \subset \mathrm{SL}(2, \mathbb{R})^{n} \times \mathbb{R}$, que converge para um ponto $(A, \theta)=\left(A_{1}, \ldots, A_{n}, \theta\right)$, temos $\theta_{j} \rightarrow \theta$ e $A_{j_{k}} \rightarrow A_{k}$ para todo $k=1, \ldots, n$, quando $j \rightarrow \infty$, deste modo, $\mathrm{SL}(2, \mathbb{R}) \ni A_{j_{n}} R_{\theta_{j}} \ldots A_{j_{1}} R_{\theta_{j}}$ converge para $A_{n} R_{\theta} \ldots A_{1} R_{\theta}$ quando $j \rightarrow \infty$, e pela continuidade das funções log e $\rho$ temos

$$
\log \rho\left(A_{j_{n}} R_{\theta_{j}} \ldots A_{j_{1}} R_{\theta_{j}}\right) \longrightarrow \log \rho\left(A_{n} R_{\theta} \ldots A_{1} R_{\theta}\right)
$$

o que prova a continuidade da função $\tilde{f}$. Note que para $A \in \operatorname{SL}(2, \mathbb{R})^{n}$ e $\theta \in \mathbb{R}$ temos

$$
f(A)=\int_{0}^{2 \pi} \tilde{f}(A, \theta) d \theta .
$$

Considere a sequência $\left(X_{j}\right)_{j \in \mathbb{N}}=\left(X_{j_{1}}, \ldots, X_{j_{n}}\right) \subset \mathrm{SL}(2, \mathbb{R})^{n}$ tal que $\lim _{j \rightarrow \infty} X_{j}=X=\left(X^{\prime}{ }_{1}, \ldots, X_{n}^{\prime}\right) \in \mathrm{SL}(2, \mathbb{R})^{n}$, portanto é suficiente provar 
que $\lim _{j \rightarrow \infty} f\left(X_{j}\right)=f(X)$, ou seja,

$\lim _{j \rightarrow \infty} \int_{0}^{2 \pi} \tilde{f}\left(X_{j}, \theta\right) d \theta=\lim _{j \rightarrow \infty} f\left(X_{j}\right)=f(X)=\int_{0}^{2 \pi} \tilde{f}(X, \theta) d \theta=\int_{0}^{2 \pi} \lim _{j \rightarrow \infty} \tilde{f}\left(X_{j}, \theta\right) d \theta$,

em que a última igualdade já segue pela continuidade de $\tilde{f}$. Defina para cada $j \in \mathbb{N}$

$$
\begin{array}{rlrl}
g_{j}: \mathbb{R} & \longrightarrow \mathbb{R} & g & g: \mathbb{R} \\
\theta & \longmapsto g_{j}(\theta)=\tilde{f}\left(X_{j}, \theta\right) & \theta & \longmapsto g(\theta)=\tilde{f}(X, \theta) .
\end{array}
$$

então como podemos observar $\lim _{j \rightarrow \infty} g_{j}(\theta)=g(\theta)$, e perceba que é suficiente provar

$$
\lim _{j \rightarrow \infty} \int_{0}^{2 \pi} g_{j}(\theta) d \theta=\int_{0}^{2 \pi} \lim _{j \rightarrow \infty} g_{j}(\theta) .
$$

Considere o espaço de medida $(\mathbb{R}, \mathcal{M}(\mathbb{R}), \mu)$, em que $\mu$ é a medida de lebesgue na reta e $\mathcal{M}(\mathbb{R})$ o conjunto dos mensuráveis à Lebesgue. Como já vimos, as funções $g_{j}$ e $g$ são contínuas para cada $j \in \mathbb{N}$ e como $\mathcal{M}(\mathbb{R})$ é $\sigma$-álgebra de Borel, as funções $g_{j}$ e $g$ são funções $\mu$-mensuráveis para todo $j \in \mathbb{N}$, além disso, já que são integráveis à Riemann, então são integráveis à Lebesgue. Sabemos também que a função característica $\mathbb{1}_{[0,2 \pi]}$ é $\mu$-mensurável e $\mu$-integrável, portanto as funções $g_{j} \cdot \mathbb{1}_{[0,2 \pi]}$ e $g \cdot \mathbb{1}_{[0,2 \pi]}$ são $\mu$-mensuráveis, e ainda temos que $g_{j} \cdot \mathbb{1}_{[0,2 \pi]}: \mathbb{R} \longrightarrow \mathbb{R}$, dada por $\left(g_{j} \cdot \mathbb{1}_{[0,2 \pi]}\right)(\theta)=g_{j}(\theta) \mathbb{1}_{[0,2 \pi]}(\theta)$ é uma função $\mu$-integrável para todo $j \in \mathbb{N}$. Note ainda que $g_{j} \cdot \mathbb{1}_{[0,2 \pi]}$ é contínua em $[0,2 \pi]$, que é um compacto e deste modo existe $M<\infty$ o qual para a função

$$
\begin{aligned}
h: \mathbb{R} & \longrightarrow \mathbb{R} \\
\theta & \longmapsto M \mathbb{1}_{[0,2 \pi]}(\theta)
\end{aligned}
$$

é tal que $\left|g_{j}(\theta) \mathbb{1}_{[0,2 \pi]}\right| \leq h(\theta)$ para todo $j \in \mathbb{N}$ e para todo $\theta \in \mathbb{R}$. Portanto, pelo Teorema da Convergência Dominada temos que $g \cdot \mathbb{1}_{[0,2 \pi]}$ é $\mu$-integrável e

$$
\begin{aligned}
\lim _{j \rightarrow \infty} \int_{0}^{2 \pi} g_{j}(\theta) d \theta & =\lim _{j \rightarrow \infty} \int_{\mathbb{R}} g_{j}(x) \mathbb{1}_{[0,2 \pi]} d \mu(x) \\
& =\int_{\mathbb{R}} g(x) \mathbb{1}_{[0,2 \pi]}(x) d \mu(x)=\int_{0}^{2 \pi} g(\theta) d \theta,
\end{aligned}
$$

o que prova a continuidade da função $f$.

Prosseguindo, o resultado a seguir também se dedica a elucidar a continuidade de uma função em particular, neste caso a função está definida sobre o espaço $\operatorname{PSL}(2, \mathbb{R}) \backslash\{[I]\}$, e em função do valor absoluto do traço da matriz 
avaliada, de modo a apresentar um comportamento para classes de matrizes elípticas e parabólicas, e outro para matrizes hiperbólicas. É relevante mencionarmos que para uma matriz qualquer $A \in \operatorname{Mat}(2, \mathbb{R}) \operatorname{temos} \operatorname{det} A=\operatorname{det}(-A)$ e $\operatorname{tr}(-A)=-\operatorname{tr} A$, assim $|\operatorname{tr} A|=|\operatorname{tr}(-A)|$, o que nos permite afirmar que a função $|\operatorname{tr}|$ está bem definida em $\operatorname{PSL}(2, \mathbb{R})$, mais ainda, sendo $v, w$ os autovetores de $A$ associados aos autovalores $\lambda$ e $\frac{1}{\lambda}$, respectivamente, temos que $-A$ possui os mesmos autovetores $v$ e $w$, neste caso, associados aos autovalores $-\lambda$ e $-\frac{1}{\lambda}$, respectivamente. Deste modo, perceba que faz sentido definirmos $F$ da maneira como mencionamos no enunciado, de certa maneira tomando representantes em cada classe.

Lema 4.10 Sejam $X=P S L(2, \mathbb{R}) \backslash[I]$ e $Y=\{[A] \in X:|\operatorname{tr} A| \leq 2\}$. Definimos $F: X \rightarrow S^{1}$ dada por

$$
F([A])= \begin{cases}1, & \text { se }[A] \in Y \\ \frac{v^{2}}{w^{2}}, & \text { se }[A] \in X \backslash Y\end{cases}
$$

em que $v, w \in S^{1} \subset \mathbb{C}$ são autovetores de $A$ normalizados, com autodireções $\pm v, \pm w$ e autovalores associados $\lambda e \frac{1}{\lambda}$, respectivamente, com $|\lambda|>1$. Então, a função $F$ é contínua em $X$.

Prova. Além do que foi discutido antes de abordarmos este resultado, um outro fato relevante é que, se definirmos $\tilde{F}: \mathrm{SL}(2, \mathbb{R}) \backslash\{I,-I\} \rightarrow S^{1}$, e provarmos a continuidade desta aplicação, então para qualquer $U \subset \tilde{F}(\mathrm{SL}(2, \mathbb{R}) \backslash\{I,-I\})$ aberto em $S^{1}$, teremos $\tilde{F}^{-1}(U) \subset \operatorname{SL}(2, \mathbb{R}) \backslash\{I,-I\}$ aberto em $\operatorname{SL}(2, \mathbb{R}) \backslash$ $\{I,-I\}$, consequentemente para a aplicação $F: \operatorname{PSL}(2, \mathbb{R}) \backslash\{[I]\} \rightarrow S^{1}$ teremos $F^{-1}(U) \subset \operatorname{PSL}(2, \mathbb{R}) \backslash\{[I]\}$ aberto em $\operatorname{PSL}(2, \mathbb{R}) \backslash\{[I]\}$, com respeito a topologia quociente, justamente pelo modo como definimos os abertos nesta topologia, de modo a implicar a continuidade de $F$. Desta maneira, focaremos em provar a continuidade da função definida sobre o espaço SL $(2, \mathbb{R}) \backslash\{I,-I\}$, contudo denotaremos a aplicação por $F$, apenas com o intuito de não carregarmos a notação e os conjuntos $X$ e $Y$ como subconjuntos de $\operatorname{SL}(2, \mathbb{R}) \backslash\{I,-I\}$.

A ideia desta prova consiste em definirmos $F$ de forma explícita em mais casos específicos, e não somente para $Y$ e $X \backslash Y$, para que então possamos tratar de sua continuidade. No conjunto aberto $\{A \in X:|\operatorname{tr} A|<2\}, F$ é constante, portanto é claramente contínua neste conjunto. Nos resta então provar a continuidade de $F$ no conjunto $X \backslash Y=\{A \in X:|\operatorname{tr} A|>2\}$ e ainda na fronteira dos conjuntos mencionados, ou seja, em $\{A \in X:|\operatorname{tr} A|=2\}$. 
Considere $v=v_{1}+i v_{2}$ e $w=w_{1}+i w_{2}$ e seja $A=\left(\begin{array}{ll}a & b \\ c & d\end{array}\right) \in X$, portanto sabemos que os autovalores de $A$ são da forma $\lambda$ e $\frac{1}{\lambda}$. Se temos $|\lambda|>1$, então $(A-\lambda I) v=0$ e $\left(A-\frac{1}{\lambda} I\right) w=0$, assim de forma geral obtemos

$$
\begin{aligned}
(A-\lambda I) v=0 & \Rightarrow \frac{v}{|v|}= \pm \frac{(b+d-\lambda)+i(\lambda-a-c)}{\left((b+d-\lambda)^{2}+(\lambda-a-c)^{2}\right)^{\frac{1}{2}}} \\
& \Rightarrow\left(\frac{v}{|v|}\right)^{2}=\frac{((b+d-\lambda)+i(\lambda-a-c))^{2}}{(b+d-\lambda)^{2}+(\lambda-a-c)^{2}}
\end{aligned}
$$

e

$$
\begin{aligned}
\left(A-\frac{1}{\lambda} I\right) w=0 & \Rightarrow \frac{w}{|w|}= \pm \frac{\left(b+d-\frac{1}{\lambda}\right)+i\left(\frac{1}{\lambda}-a-c\right)}{\left(\left(b+d-\frac{1}{\lambda}\right)^{2}+\left(\frac{1}{\lambda}-a-c\right)^{2}\right)^{\frac{1}{2}}} \\
& \Rightarrow\left(\frac{w}{|w|}\right)^{2}=\frac{\left(\left(b+d-\frac{1}{\lambda}\right)+i\left(\frac{1}{\lambda}-a-c\right)\right)^{2}}{\left(b+d-\frac{1}{\lambda}\right)^{2}+\left(\frac{1}{\lambda}-a-c\right)^{2}} .
\end{aligned}
$$

E portanto

$$
F(A)=\frac{\left(\frac{v}{|v|}\right)^{2}}{\left(\frac{w}{|w|}\right)^{2}}=\frac{((b+d-\lambda)+i(\lambda-a-c))^{2}\left(\left(b+d-\frac{1}{\lambda}\right)^{2}+\left(\frac{1}{\lambda}-a-c\right)^{2}\right)}{\left((b+d-\lambda)^{2}+(\lambda-a-c)^{2}\right)\left(\left(b+d-\frac{1}{\lambda}\right)+i\left(\frac{1}{\lambda}-a-c\right)\right)^{2}} .
$$

Além disso, grosso modo, $A$ pode assumir quatro formas distintas:

1. Caso em que $b=c=0$.

Temos $A=\left(\begin{array}{ll}a & 0 \\ 0 & d\end{array}\right)=\left(\begin{array}{cc}a & 0 \\ 0 & \frac{1}{a}\end{array}\right)$, ou seja, $d=\frac{1}{a}$, e portanto os autovalores de $A$ são justamente $a$ e $\frac{1}{a}$, nos fornecendo então duas novas possibilidades $|a|>1$ ou $|a| \leq 1$.

1.1 Se $|a|>1$, então $\lambda=a$, assim $v$ é associado à $a$, enquanto $w$ é associado à $\frac{1}{a}$, e deste modo segue que

$$
\frac{v}{|v|}= \pm \frac{\left(\frac{1}{a}-a\right)}{\left(\left(\frac{1}{a}-a\right)^{2}\right)^{\frac{1}{2}}}= \pm 1 \quad \text { e } \quad \frac{w}{|w|}= \pm \frac{i\left(\frac{1}{a}-a\right)}{\left(\left(\frac{1}{a}-a\right)^{2}\right)^{\frac{1}{2}}}= \pm i
$$

e portanto

$$
F(A)=\frac{( \pm 1)^{2}}{( \pm i)^{2}}=-1
$$


1.2 Se $|a| \leq 1$, então $\lambda=\frac{1}{a}$, assim $v$ é associado à $\frac{1}{a}$, enquanto $w$ é associado à $a$, e deste modo segue que

$$
\frac{v}{|v|}= \pm \frac{i\left(\frac{1}{a}-a\right)}{\left.\left(\frac{1}{a}-a\right)^{2}\right)^{\frac{1}{2}}}= \pm i \quad \text { e } \quad \frac{w}{|w|}= \pm \frac{\left(\frac{1}{a}-a\right)}{\left(\left(\frac{1}{a}-a\right)^{2}\right)^{\frac{1}{2}}}= \pm 1
$$

e portanto

$$
F(A)=\frac{( \pm i)^{2}}{( \pm 1)^{2}}=-1
$$

Então, de todo modo, para o caso 1 temos $F=-1$.

2. Caso em que $b=0$ e $c \neq 0$.

Temos $A=\left(\begin{array}{ll}a & 0 \\ c & d\end{array}\right)=\left(\begin{array}{ll}a & 0 \\ c & \frac{1}{a}\end{array}\right)$, ou seja, $d=\frac{1}{a}$, e continuamos a ter os autovalores de $A$ dados por $a$ e $\frac{1}{a}$, que ainda nos fornecem duas novas possibilidades $|a|>1$ ou $|a| \leq 1$.

2.1 Se $|a|>1$, então $\lambda=a$, assim $v$ é associado à $a$, enquanto $w$ é associado à $\frac{1}{a}$, e deste modo segue que

$$
\frac{v}{|v|}= \pm \frac{\left(\frac{1}{a}-a\right)-i c}{\left(\left(\frac{1}{a}-a\right)^{2}+c^{2}\right)^{\frac{1}{2}}} \quad \text { e } \quad \frac{w}{|w|}= \pm \frac{i\left(\frac{1}{a}-a-c\right)}{\left(\left(\frac{1}{a}-a-c\right)^{2}\right)^{\frac{1}{2}}}= \pm i
$$

e portanto

$$
F(A)=\frac{\left( \pm \frac{\left(\frac{1}{a}-a\right)-i c}{\left(\left(\frac{1}{a}-a\right)^{2}+c^{2}\right)^{\frac{1}{2}}}\right)^{2}}{( \pm i)^{2}}=-\frac{\left(\left(\frac{1}{a}-a\right)-i c\right)^{2}}{\left(\frac{1}{a}-a\right)^{2}+c^{2}}
$$

2.2 Se $|a| \leq 1$, então $\lambda=\frac{1}{a}$, assim $v$ é associado à $\frac{1}{a}$, enquanto $w$ é associado à $a$, e deste modo segue que

$$
\frac{v}{|v|}= \pm \frac{i\left(\frac{1}{a}-a-c\right)}{\left(\left(\frac{1}{a}-a-c\right)^{2}\right)^{\frac{1}{2}}}= \pm i \quad \text { e } \quad \frac{w}{|w|}= \pm \frac{\left(\frac{1}{a}-a\right)-i c}{\left(\left(\frac{1}{a}-a\right)^{2}+c^{2}\right)^{\frac{1}{2}}}
$$

e portanto

$$
F(A)=\frac{( \pm i)^{2}}{\left( \pm \frac{\left(\frac{1}{a}-a\right)-i c}{\left(\left(\frac{1}{a}-a\right)^{2}+c^{2}\right)^{\frac{1}{2}}}\right)^{2}}=-\frac{\left(\frac{1}{a}-a\right)^{2}+c^{2}}{\left(\left(\frac{1}{a}-a\right)-i c\right)^{2}}
$$

3. Caso em que $b \neq 0$ e $c=0$. 
Temos $A=\left(\begin{array}{ll}a & b \\ 0 & d\end{array}\right)=\left(\begin{array}{ll}a & b \\ 0 & \frac{1}{a}\end{array}\right)$, ou seja, $d=\frac{1}{a}$, e assim como nos demais casos, continuamos a ter $a$ e $\frac{1}{a}$ como os autovalores de $A$, os quais continuam a nos fornecer duas novas possibilidades $|a|>1$ e $|a| \leq 1$.

3.1 Se $|a|>1$, então $\lambda=a$, assim $v$ é associado à $a$, enquanto $w$ é associado à $\frac{1}{a}$, e deste modo obtemos

$$
\frac{v}{|v|}= \pm \frac{b+\frac{1}{a}-a}{\left(\left(b+\frac{1}{a}-a\right)^{2}\right)^{\frac{1}{2}}}= \pm 1 \quad \text { e } \quad \frac{w}{|w|}= \pm \frac{b+i\left(\frac{1}{a}-a\right)}{\left(b^{2}+\left(\frac{1}{a}-a\right)^{2}\right)^{\frac{1}{2}}}
$$

e portanto

$$
F(A)=\frac{( \pm 1)^{2}}{\left( \pm \frac{b+i\left(\frac{1}{a}-a\right)}{\left(b^{2}+\left(\frac{1}{a}-a\right)^{2}\right)^{\frac{1}{2}}}\right)^{2}}=\frac{b^{2}+\left(\frac{1}{a}-a\right)^{2}}{\left(b+i\left(\frac{1}{a}-a\right)\right)^{2}}
$$

3.2 Se $|a| \leq 1$, então $\lambda=\frac{1}{a}$, assim $v$ é associado à $\frac{1}{a}$, enquanto $w$ é associado à $a$, e deste modo segue que

$$
\frac{v}{|v|}= \pm \frac{b+i\left(\frac{1}{a}-a\right)}{\left(b^{2}+\left(\frac{1}{a}-a\right)^{2}\right)^{\frac{1}{2}}} \quad \text { e } \frac{w}{|w|}= \pm \frac{b+\frac{1}{a}-a}{\left(\left(b+\frac{1}{a}-a\right)^{2}\right)^{\frac{1}{2}}}= \pm 1
$$

e portanto

$$
F(A)=\frac{\left( \pm \frac{b+i\left(\frac{1}{a}-a\right)}{\left(b^{2}+\left(\frac{1}{a}-a\right)^{2}\right)^{\frac{1}{2}}}\right)^{2}}{( \pm 1)^{2}}=\frac{\left(b+i\left(\frac{1}{a}-a\right)\right)^{2}}{b^{2}+\left(\frac{1}{a}-a\right)^{2}} .
$$

4. Caso em que $b \neq 0$ e $c \neq 0$.

Para este caso, o qual é o mais geral, as contas foram feitas de forma arbitrária antes de começarmos a estudar caso à caso, e como vimos temos uma matriz $A=\left(\begin{array}{ll}a & b \\ c & d\end{array}\right) \in X$ com autovalores $\lambda$ e $\frac{1}{\lambda}$, o que também nos fornece duas outras possibilidades

4.1 Se $\lambda>1$, então $v$ é associado à $\lambda$, enquanto $w$ é associado à $\frac{1}{\lambda} \mathrm{e}$ as contas são exatamente as mesmas feitas no início da prova, de 
modo a obtermos

$$
\begin{aligned}
\frac{v}{|v|} & = \pm \frac{(b+d-\lambda)+i(\lambda-a-c)}{\left((b+d-\lambda)^{2}+(\lambda-a-c)^{2}\right)^{\frac{1}{2}}} \\
\frac{w}{|w|} & = \pm \frac{\left(b+d-\frac{1}{\lambda}\right)+i\left(\frac{1}{\lambda}-a-c\right)}{\left(\left(b+d-\frac{1}{\lambda}\right)^{2}+\left(\frac{1}{\lambda}-a-c\right)^{2}\right)^{\frac{1}{2}}}
\end{aligned}
$$

e portanto

$$
F(A)=\frac{((b+d-\lambda)+i(\lambda-a-c))^{2}\left(\left(b+d-\frac{1}{\lambda}\right)^{2}+\left(\frac{1}{\lambda}-a-c\right)^{2}\right)}{\left((b+d-\lambda)^{2}+\left(\frac{1}{\lambda}-a-c\right)^{2}\right)\left(\left(b+d-\frac{1}{\lambda}\right)+i\left(\frac{1}{\lambda}-a-c\right)\right)^{2}} .
$$

4.2 Se $|\lambda| \leq 1$, então $v$ é associado à $\frac{1}{\lambda}$, enquanto $w$ é associado à $\lambda$, e deste modo as contas seguem de forma idêntica à apresentada acima, porém invertendo os papéis de $\lambda$ e $\frac{1}{\lambda}$, de modo a obtermos

$$
\begin{aligned}
\frac{v}{|v|} & = \pm \frac{\left(b+d-\frac{1}{\lambda}\right)+i\left(\frac{1}{\lambda}-a-c\right)}{\left(\left(b+d-\frac{1}{\lambda}\right)^{2}+\left(\frac{1}{\lambda}-a-c\right)^{2}\right)^{\frac{1}{2}}} \mathrm{e} \\
\frac{w}{|w|} & = \pm \frac{(b+d-\lambda)+i(\lambda-a-c)}{\left((b+d-\lambda)^{2}+(\lambda-a-c)^{2}\right)^{\frac{1}{2}}}
\end{aligned}
$$

e portanto

$$
F(A)=\frac{\left(\left(b+d-\frac{1}{\lambda}\right)+i\left(\frac{1}{\lambda}-a-c\right)\right)^{2}\left((b+d-\lambda)^{2}+(\lambda-a-c)^{2}\right)}{\left(\left(b+d-\frac{1}{\lambda}\right)^{2}+(\lambda-a-c)^{2}\right)((b+d-\lambda)+i(\lambda-a-c))^{2}} .
$$

Agora que pudemos definir a função $F$ de forma explícita para uma matriz $A=\left(\begin{array}{ll}a & b \\ c & d\end{array}\right) \in X$, a qual, como vimos, admite diversas formas distintas, falaremos de fato da continuidade de $F$ no conjunto $X \backslash Y$.

Seja $\left(A_{n}\right)_{n \in \mathbb{N}}=\left(\begin{array}{ll}a_{n} & b_{n} \\ c_{n} & d_{n}\end{array}\right) \subset X \backslash Y$ tal que $A_{n} \rightarrow A=\left(\begin{array}{ll}a & b \\ c & d\end{array}\right) \in X$, quando $n \rightarrow \infty$, ou seja, temos $a_{n} \rightarrow a, b_{n} \rightarrow b, c_{n} \rightarrow c, d_{n} \rightarrow d$ quando $n \rightarrow \infty$. Claramente não podemos ter $|\operatorname{tr} A|<2$, até pela continuidade da função traço, contudo nos restam as possibilidades $|\operatorname{tr} A|>2$ e $|\operatorname{tr} A|=2$, então analisaremos ambos os casos. Para todas as matrizes trabalhadas sabemos que seus autovalores tem a forma $\lambda, \frac{1}{\lambda}$, deste modo, denotaremos por $\lambda(M), \frac{1}{\lambda(M)}$ os autovalores de uma matriz $M$, além disso, como vimos durante a prova, a imagem da aplicação $F$ é definida em função dos autovalores da matriz avaliada, portanto é pertinente lembrarmos que no Lema 4.8, foi provado que os autovalores de uma matriz variam continuamente a medida que variamos seus coeficientes, portanto, grosso modo, a função $M \mapsto \lambda(M)$ é contínua, e 
$\operatorname{assim} \lambda\left(A_{n}\right) \rightarrow \lambda(A)$ quando $n \rightarrow \infty$. Considere por $v_{n}$ e $w_{n}$ os autovalores de $A_{n}$ associados ao autovalor com norma maior que 1 e norma menor ou igual à 1 , respectivamente.

- $|\operatorname{tr} A|>2$.

Afim de evitarmos prolongar mais que o necessário a prova em questão, apenas observaremos que neste caso a continuidade se dará de forma clara, pois tomando a referida sequência de matrizes $\left(A_{n}\right)_{n \in \mathbb{N}}$, obtemos as sequências respectivas a cada termo da matriz, $\left(a_{n}\right)_{n \in \mathbb{N}},\left(b_{n}\right)_{n \in \mathbb{N}},\left(c_{n}\right)_{n \in \mathbb{N}}$, $\left(d_{n}\right)_{n \in \mathbb{N}}$, e uma vez que $F$ é definida diretamente em função destes coeficientes e dos autovalores da matriz avaliada, o qual já mostramos que variam continuamente à medida que variamos continuamente os coeficientes desta matriz, e portanto a convergêcia $F\left(A_{n}\right) \rightarrow F(A)$ fica evidente.

- $|\operatorname{tr} A|=2$.

Neste caso temos $F(A)=1$. Sabemos que, grosso modo, os conjuntos os quais a sequência $\left(A_{n}\right)_{n \in \mathbb{N}}$ possui um número finito de termos não interferirão no resultado, desta forma, sem perda de generalidade, assumiremos que $\left(A_{n}\right)_{n \in \mathbb{N}}$ está inteiramente contida em cada um dos casos a seguir:

1. Considere $\left(A_{n}\right)_{n \in \mathbb{N}}$ de modo que $b_{n}=c_{n}=0$ para todo $n \in \mathbb{N}$, ou seja, $A_{n}=\left(\begin{array}{cc}a_{n} & 0 \\ 0 & \frac{1}{a_{n}}\end{array}\right)$ para todo $n \in \mathbb{N}$. Então devemos ter $A=\left(\begin{array}{cc}a & 0 \\ 0 & \frac{1}{a}\end{array}\right)$ e já que $\left|a+\frac{1}{a}\right|=|\operatorname{tr} A|=2$, temos $a= \pm 1$, e consequentemente deveríamos ter $A= \pm I$. Porém tal fato não é possível, já que $X=\operatorname{PSL}(2, \mathbb{R}) \backslash\{[I]\}$. Portanto, $\left(A_{n}\right)_{n \in \mathbb{N}}$ não pode ter infinitos termos com a forma apresentada.

2. Considere $\left(A_{n}\right)_{n \in \mathbb{N}}$ de modo que $b_{n}=0$ e $c_{n} \neq 0$ para todo $n \in \mathbb{N}$, ou seja, $A_{n}=\left(\begin{array}{cc}a_{n} & 0 \\ c_{n} & \frac{1}{a_{n}}\end{array}\right)$ para todo $n \in \mathbb{N}$. Então devemos ter $A=\left(\begin{array}{cc}a & 0 \\ c & \frac{1}{a}\end{array}\right)$ e já que $\left|a+\frac{1}{a}\right|=|\operatorname{tr} A|=2$, temos $a= \pm 1$, e consequentemente devemos ter $A=\left(\begin{array}{cc} \pm 1 & 0 \\ c & \pm 1\end{array}\right)$, sem perda de generalidade assumiremos $a=1$, pois o caso em que $a=-1$ é análogo. Contudo temos duas novas possibilidade $\left|a_{n}\right|>1$ ou $\left|a_{n}\right| \leq 1$, para todo $n \geq m$, para algum $m \in \mathbb{N}$ suficientemente grande, já que no caso em que temos infinitos termos $\left|a_{n}\right|>1$ e 
infinitos termos $\left|a_{n}\right| \leq 1$, estas duas sequências obrigatóriamente convergirão para 1 (ou -1 no caso análogo).

2.1 Se $\left|a_{n}\right|>1$ para todo $n$ suficientemente grande, então $v_{n}$ é associado à $a_{n}$, enquanto que $w_{n}$ é associado à $\frac{1}{a_{n}}$, para todo $n$ suficientemente grande, e portanto

$$
\begin{aligned}
\lim _{n \rightarrow \infty} F\left(A_{n}\right) & =\lim _{n \rightarrow \infty}-\frac{\left(\left(\frac{1}{a_{n}}-a_{n}\right)-i c_{n}\right)^{2}}{\left(\frac{1}{a_{n}}-a_{n}\right)^{2}+c_{n}^{2}} \\
& =-\frac{\left((1-1)+i c_{n}\right)^{2}}{(1-1)^{2}+c_{n}^{2}}=\frac{c^{2}}{c^{2}}=1=F(A) .
\end{aligned}
$$

2.2 Se $\left|a_{n}\right| \leq 1$ para todo $n$ suficientemente grande, então $v_{n}$ é associado à $\frac{1}{a_{n}}$, enquanto que $w_{n}$ é associado à $a_{n}$, para todo $n$ suficientemente grande, e portanto

$$
\begin{aligned}
\lim _{n \rightarrow \infty} F\left(A_{n}\right) & =\lim _{n \rightarrow \infty}-\frac{\left(\frac{1}{a_{n}}-a_{n}\right)^{2}+c_{n}^{2}}{\left(\left(\frac{1}{a_{n}}-a_{n}\right)-i c_{n}\right)^{2}} \\
& =-\frac{(1-1)^{2}+c^{2}}{((1-1)-i c)^{2}}=1=F(A) .
\end{aligned}
$$

3. Considere $\left(A_{n}\right)_{n \in \mathbb{N}}$ de modo que $b_{n} \neq 0$ e $c_{n}=0$ para todo $n \in \mathbb{N}$, ou seja, $A_{n}=\left(\begin{array}{cc}a_{n} & b_{n} \\ 0 & \frac{1}{a_{n}}\end{array}\right)$ para todo $n \in \mathbb{N}$. Então devemos ter $A=\left(\begin{array}{cc}a & b \\ 0 & \frac{1}{a}\end{array}\right)$ e já que $\left|a+\frac{1}{a}\right|=|\operatorname{tr} A|=2, \operatorname{temos} a= \pm 1$, e consequentemente devemos ter $A=\left(\begin{array}{cc} \pm 1 & b \\ 0 & \pm 1\end{array}\right)$, sem perda de generalidade assumiremos $a=1$, pois o caso em que $a=-1$ é análogo. Contudo temos duas novas possibilidade $\left|a_{n}\right|>1$ ou $\left|a_{n}\right| \leq 1$, para todo $n \geq m$, para algum $m \in \mathbb{N}$ suficientemente grande, já que no caso em que temos infinitos termos $\left|a_{n}\right|>1$ e infinitos termos $\left|a_{n}\right| \leq 1$, estas duas sequências obrigatóriamente convergirão para 1 (ou -1 no caso análogo).

3.1 Se $\left|a_{n}\right|>1$ para todo $n$ suficientemente grande, então $v_{n}$ é associado à $a_{n}$, enquanto que $w_{n}$ é associado à $\frac{1}{a_{n}}$, para todo $n$ suficientemente grande, e portanto

$$
\begin{aligned}
\lim _{n \rightarrow \infty} F\left(A_{n}\right) & =\lim _{n \rightarrow \infty} \frac{b_{n}^{2}+\left(\frac{1}{a_{n}}-a_{n}\right)^{2}}{\left(b_{n}+i\left(\frac{1}{a_{n}}-a_{n}\right)\right)^{2}} \\
& =\frac{b^{2}+(1-1)^{2}}{(b+i(1-1))^{2}}=1=F(A) .
\end{aligned}
$$


3.2 Se $\left|a_{n}\right| \leq 1$ para todo $n$ suficientemente grande, então $v_{n}$ é associado à $\frac{1}{a_{n}}$, enquanto que $w_{n}$ é associado à $a_{n}$, para todo $n$ suficientemente grande, e portanto

$$
\begin{aligned}
\lim _{n \rightarrow \infty} F\left(A_{n}\right) & =\lim _{n \rightarrow \infty} \frac{\left(b_{n}+i\left(\frac{1}{a_{n}}-a_{n}\right)\right)^{2}}{b_{n}^{2}+\left(\frac{1}{a_{n}}-a_{n}\right)^{2}} \\
& =\frac{(b+i(1-1))^{2}}{b^{2}+(1-1)^{2}}=1=F(A) .
\end{aligned}
$$

4. Considere $\left(A_{n}\right)_{n \in \mathbb{N}}$ de modo que $b_{n} \neq 0$ e $c_{n} \neq 0$ para todo $n \in \mathbb{N}$. Lembre-se, temos $A=\left(\begin{array}{ll}a & b \\ c & d\end{array}\right)$ em que $|a+d|=|\operatorname{tr} A|=2$, assim $d= \pm 2-a$ e portanto

$$
\frac{\operatorname{tr} A \pm \sqrt{(\operatorname{tr} A)^{2}-4 \operatorname{det} A}}{2}=\frac{a \pm 2-a \pm \sqrt{(a \pm 2-a)^{2}-4}}{2}= \pm 1
$$

Deste modo temos $\lambda(A)=\frac{1}{\lambda(A)}= \pm 1$ e consequentemente $\lim _{n \rightarrow \infty} \lambda\left(A_{n}\right)=\lambda(A)= \pm 1$. De modo análogo ao que foi discutido nos demais casos, vamos sem perda de generalidade assumir $\lambda(A)=1$, pois o caso em que $\lambda(A)=-1$ é análogo. Contudo temos duas novas possibilidades $\left|\lambda\left(A_{n}\right)\right|>1$ ou $\left|\lambda\left(A_{n}\right)\right| \leq 1$, para todo $n \geq m$, para algum $m \in \mathbb{N}$ suficientemente grande, já que no caso em que temos infinitos termos $\left|\lambda\left(A_{n}\right)\right|>1$ e infinitos termos $\left|\lambda\left(A_{n}\right)\right| \leq 1$, estas duas sequências obrigatóriamente convergirão para 1 (ou -1 no caso análogo).

4.1 Se $\left|\lambda\left(A_{n}\right)\right|>1$ para todo $n$ suficientemente grande, então $v_{n}$ é associado à $\lambda\left(A_{n}\right)$, enquanto que $w_{n}$ é associado à $\frac{1}{\lambda\left(A_{n}\right)}$, para todo $n$ suficientemente grande, assim segue-se

$$
\begin{aligned}
\lim _{n \rightarrow \infty} F\left(A_{n}\right)= & \lim _{n \rightarrow \infty} \frac{\left(\left(b_{n}+d_{n}-\lambda\left(A_{n}\right)\right)+i\left(\lambda\left(A_{n}\right)-a_{n}-c_{n}\right)\right)^{2}}{\left(\left(b_{n}+d_{n}-\lambda\left(A_{n}\right)\right)^{2}+\left(\lambda\left(A_{n}\right)-a_{n}-c_{n}\right)^{2}\right)} \\
& \lim _{n \rightarrow \infty} \frac{\left(\left(b_{n}+d_{n}-\frac{1}{\lambda\left(A_{n}\right)}\right)^{2}+\left(\frac{1}{\lambda\left(A_{n}\right)}-a_{n}-c_{n}\right)^{2}\right)}{\left(\left(b_{n}+d_{n}-\frac{1}{\lambda\left(A_{n}\right)}\right)+i\left(\frac{1}{\lambda\left(A_{n}\right)}-a_{n}-c_{n}\right)\right)^{2}} \\
= & \frac{((b+d-\lambda(A))+i(\lambda(A)-a-c))^{2}}{\left((b+d-\lambda(A))^{2}+(\lambda(A)-a-c)^{2}\right)} \\
& \frac{\left(\left(b+d-\frac{1}{\lambda(A)}\right)^{2}+\left(\frac{1}{\lambda(A)}-a-c\right)^{2}\right)}{\left(\left(b+d-\frac{1}{\lambda(A)}\right)+i\left(\frac{1}{\lambda(A)}-a-c\right)\right)^{2}}
\end{aligned}
$$




$$
\begin{aligned}
= & \frac{((b+d-\lambda(A))+i(\lambda(A)-a-c))^{2}}{\left((b+d-\lambda(A))^{2}+(\lambda(A)-a-c)^{2}\right)} \\
& \frac{\left((b+d-\lambda(A))^{2}+(\lambda(A)-a-c)^{2}\right)}{((b+d-\lambda(A))+i(\lambda(A)-a-c))^{2}}=1=F(A) .
\end{aligned}
$$

4.2 Se $\left|\lambda\left(A_{n}\right)\right| \leq 1$ para todo $n$ suficientemente grande, então $v_{n}$ é associado à $\frac{1}{\lambda\left(A_{n}\right)}$, enquanto que $w_{n}$ é associado à $\lambda\left(A_{n}\right)$, para todo $n$ suficientemente grande. Como já foi apresentado, o caso em questão é exatamente o mesmo do item 4.1, exceto pela inversão dos papéis de $\lambda(A)$ e $\frac{1}{\lambda(A)}$. Contudo, temos $\lambda(A)= \pm 1$, deste modo $\lambda(A)=\frac{1}{\lambda(A)}$ e portanto o caso torna-se o mesmo do anterior, assim sendo

$$
\lim _{n \rightarrow \infty} F\left(A_{n}\right)=1=F(A)
$$

Desta maneira, $F$, de fato, trata-se de uma função contínua.

Ainda com respeito a função apresentada acima, provaremos no lema a seguir duas propriedades, as quais serão bastante úteis em provas futuras.

\section{Proposição 4.11 (Propriedades da função $F$ )}

Considere a função F como definida no lema anterior, Lema 4.10. Então para qualquer matriz $A \in S L(2, \mathbb{R}) \backslash\{I,-I\}$ e qualquer matriz de rotação $R_{\theta}$ temos

(i) $F\left(\left[A^{T}\right]\right)=\overline{F([A])}$, em que $A^{T}$ é a matriz transposta de $A$.

(ii) $F\left(\left[R_{\theta}^{-1} A R_{\theta}\right]\right)=F([A])$.

Prova. Até pelo que foi argumentado no texto anterior ao resultado acima, faz sentido lidarmos apenas com representantes das classes.

(i) Para o caso em que $[A] \in Y$, temos $\left[A^{T}\right] \in Y$, já que $\operatorname{tr} A^{T}=\operatorname{tr} A$, portanto $F([A])=1=F\left(\left[A^{T}\right]\right)$ e portanto $\overline{F([A])}=1=F\left(\left[A^{T}\right]\right)$. Veremos então o caso em que $[A] \in X \backslash Y$. Sejam $v, w \in S^{1} \in \mathbb{C}$ os autovetores de $A$ e $v^{*}, w^{*}$ os autovetores de $A^{T} \operatorname{associados}$ à $\lambda, \frac{1}{\lambda}$ respectivamente, com $|\lambda|>1$. Note que

$$
\frac{1}{\lambda}\left\langle v, w^{*}\right\rangle=\left\langle v, \frac{1}{\lambda} w^{*}\right\rangle=\left\langle v, A^{T} w^{*}\right\rangle=\left\langle A v, w^{*}\right\rangle=\left\langle\lambda v, w^{*}\right\rangle=\lambda\left\langle v, w^{*}\right\rangle .
$$

Como $A$ é uma matriz hiperbólica, $|\operatorname{tr} A|>2$, então $\lambda \neq 1$ e consequentemente $\lambda \neq \frac{1}{\lambda}$ e portanto temos $v \perp w^{*}$, de modo análogo obtemos 
$v^{*} \perp w$. Então se temos $v=e^{i \theta}$ e $w=e^{i \varphi}$ para $\theta, \varphi \in[-\pi, \pi]$, sabemos que $v^{*}=e^{i \theta^{*}}$ e $w^{*}=e^{i \varphi^{*}}$ tais que $\left|\theta-\varphi^{*}\right|= \pm \frac{\pi}{2}$ e $\left|\theta^{*}-\varphi\right|= \pm \frac{\pi}{2} \mathrm{e}$ como $\overline{F([A])}=\left(\frac{\bar{v}}{\bar{w}}\right)^{2}$ e $F\left(\left[A^{T}\right]\right)=\left(\frac{v^{*}}{w^{*}}\right)^{2}$, para que possamos obter a propriedade, é suficiente termos $\frac{\bar{v}}{\bar{w}}= \pm \frac{v^{*}}{w^{*}}$, ou seja, $\bar{v} w^{*}= \pm v^{*} \bar{w}$, em que estamos lidando com o produto usual definido sobre o corpo $\mathbb{C}$. Temos $\bar{v} w^{*}=e^{-i \theta} e^{i \varphi^{*}}=e^{i\left(\varphi^{*}-\theta\right)}$ e $v^{*} \bar{w}=e^{i \theta^{*}} e^{-i \varphi}=e^{i\left(\theta^{*}-\varphi\right)}$, em que $\varphi^{*}-\theta=\theta^{*}-\varphi+k \pi$ para $k \in \mathbb{Z}$, se $k$ é um múltiplo de 2 obtemos $\bar{v} w^{*}=v^{*} \bar{w}$, caso contrário temos

$$
\bar{v} w^{*}=e^{i\left(\varphi^{*}-\theta\right)}=e^{i\left(\theta^{*}-\varphi\right)} e^{i k \pi}=e^{i\left(\theta^{*}-\varphi\right)} e^{i \pi}=-e^{i\left(\theta^{*}-\varphi\right)}=-v^{*} \bar{w},
$$

e portanto a propriedade está provada.

(ii) Note que $R_{\theta}^{-1}=R_{-\theta}$, como sabemos $\operatorname{det}\left(R_{-\theta} A R_{\theta}\right)=\operatorname{det}(A) \mathrm{e}$ $\operatorname{tr}\left(R_{-\theta} A R_{\theta}\right)=\operatorname{tr} A$, podemos afirmar então que os autovalores de $A \mathrm{e}$ $R_{-\theta} A R_{\theta}$ são os mesmos, mais ainda, são da forma $\lambda, \frac{1}{\lambda}$. Sejam $v, w \in \mathbb{C}$ os autovetores de $R_{-\theta} A R_{\theta}$ associados à $\lambda$ e $\frac{1}{\lambda}$, respectivamente, com $|\lambda|>1$, em que sem perda de generalidade estamos assumindo $|v|=|w|=1$. Temos

$$
\left(R_{-\theta} A R_{\theta}\right) v=\lambda v \Rightarrow\left(A R_{\theta}\right) v=\lambda R_{\theta} v \Rightarrow A\left(R_{\theta} v\right)=\lambda\left(R_{\theta} v\right)
$$

portanto $R_{\theta} v$ é um autovetor de $A$ associado à $\lambda$. Analogamente, $R_{\theta} w$ é um autovetor de $A$ associado à $\frac{1}{\lambda}$. Sabemos que existem $x, y \in \mathbb{R}$ tais que $v=e^{i x}$ e $w=e^{i y}$, então $R_{\theta} v=e^{i(x+\theta)}$ e $R_{\theta} w=e^{i(y+\theta)}$, portanto:

- Se $|\lambda|>1$.

$$
\begin{aligned}
F\left(\left[R_{-\theta} A R_{\theta}\right]\right) & =\frac{v^{2}}{w^{2}}=\frac{e^{2 i x}}{e^{2 i y}}=e^{2 i(x-y)}=e^{2 i(x+\theta-(y+\theta))} \\
& =\frac{e^{2 i(x+\theta)}}{e^{2 i(y+\theta)}}=\frac{\left(R_{\theta} v\right)^{2}}{\left(R_{\theta} w\right)^{2}}=F([A])
\end{aligned}
$$

- Se $|\lambda|<1$, o caso é análogo ao anterior.

O próximo lema a ser apresentado é um resultado obtido de forma geral sobre o espaço das matrizes complexas $2 \times 2$, dado em função do traço e do determinante da matriz em questão, que na realidade trata-se de um critério para avaliarmos quando os autovalores de uma matriz possuem mesma norma. 
Lema 4.12 Seja $C \in \operatorname{Mat}(2, \mathbb{C})$ com $\operatorname{det} C \neq 0$ e sejam $\lambda_{1}, \lambda_{2}$ os autovalores de C. Então $\left|\lambda_{1}\right|=\left|\lambda_{2}\right|$ se, e somente se,

$$
\frac{(\operatorname{tr} C)^{2}}{4 \operatorname{det} C} \in[0,1]
$$

Prova. Como $\operatorname{det} C \neq 0$, então $\lambda_{1} \neq 0$ e $\lambda_{2} \neq 0$, assim sendo, faz sentido definirmos $t=\frac{\lambda_{1}}{\lambda_{2}}$, além disso, como sabemos $\operatorname{det} C=\lambda_{1} \lambda_{2}$ e $\operatorname{tr} C=\lambda_{1}+\lambda_{2}$, portanto

$$
u:=\frac{(\operatorname{tr} C)^{2}}{4 \operatorname{det} C}=\frac{1}{4} \frac{\left(\lambda_{1}+\lambda_{2}\right)^{2}}{\lambda_{1} \lambda_{2}}=\frac{1}{4}\left(\frac{\lambda_{1}}{\lambda_{2}}+\frac{\lambda_{2}}{\lambda_{1}}+2\right)=\frac{1}{4}\left(t+t^{-1}+2\right) .
$$

Então, assumindo $t=a+i b$, para algum $a, b \in \mathbb{R}$ temos

$$
\begin{aligned}
\left|\lambda_{1}\right|=\left|\lambda_{2}\right| & \Longleftrightarrow|t|=1 \Longleftrightarrow a^{2}+b^{2}=1 \Longleftrightarrow \frac{t+t^{-1}}{2}=\frac{t^{2}+1}{2 t} \\
& =\frac{1}{2 t}\left(a^{2}-b^{2}+2 a b i+1\right)=\frac{1}{2 t}\left(a(a+i b)-b^{2}+a b i+1\right) \\
& =\frac{1}{2 t}\left(a(a+i b)-b^{2}+a b i+a^{2}+b^{2}\right)=\frac{1}{2 t}\left(a(a+i b)+a b i+a^{2}\right) \\
& =\frac{1}{2 t}(a(a+i b)+a(a+i b))=\frac{2 a t}{2 t}=a=\operatorname{Re}(t) \in[-1,1] \\
& \Longleftrightarrow u=\frac{1}{4}\left(t+t^{-1}+2\right) \in[0,1]
\end{aligned}
$$

em que $\operatorname{Re}(t)$ representa a parte real de $t$, neste caso $a$, e a última implicação segue pois $t+t^{-1} \in[-2,2] \Longleftrightarrow t+t^{-1}+2 \in[0,4] \Longleftrightarrow \frac{1}{4}\left(t+t^{-1}+2\right) \in[0,1]$. Portanto, obtemos um critério para identificar quando as normas dos autovalores de uma matrix complexa são iguais.

Para que possamos seguir e tratar de forma clara os próximos resultados, relembraremos três importantes matrizes complexas em $\operatorname{Mat}(2, \mathbb{C})$, as quais compõem o processo de complexificação de $R_{\theta}$ em que estamos interessados, para a prova de um resultado em particular, fato este que será discutido à frente. Precisaremos também definir uma função complexa racional como se segue: para todo $z \in \mathbb{C}^{*}:=\mathbb{C} \backslash\{0\}$ temos

$$
S_{z}=\left(\begin{array}{cc}
\frac{z+z^{-1}}{2} & -\frac{z-z^{-1}}{2 i} \\
\frac{z-z^{-1}}{2 i} & \frac{z+z^{-1}}{2}
\end{array}\right),
$$


e para todo $z \in \mathbb{C}$

$$
\mathscr{R}(z)=\left(\begin{array}{cc}
\frac{z^{2}+1}{2} & -\frac{z^{2}-1}{2 i} \\
\frac{z^{2}-1}{2 i} & \frac{z^{2}+1}{2}
\end{array}\right)
$$

Perceba que temos $\mathscr{R}(z)=z S_{z}$ e $S_{e^{i \theta}}=R_{\theta}$. Para $n \in \mathbb{N}, A_{1}, \ldots, A_{n}$ matrizes em $\operatorname{SL}(2, \mathbb{R})$ e $z \in \mathbb{C}$ definimos

$$
C_{z}=\prod_{j=1}^{n} A_{j} \mathscr{R}(z)=A_{n} \mathscr{R}(z) \ldots A_{1} \mathscr{R}(z)
$$

Defina a seguinte função racional

$$
\begin{aligned}
Q: \mathbb{C}^{*} & \longrightarrow \mathbb{C} \\
z & \longmapsto \frac{\operatorname{tr} C_{z}}{2 z^{n}},
\end{aligned}
$$

e considere $S:=Q^{-1}([-1,1])$.

Lema 4.13 Se $S \cap S^{1}$ tem ao menos $2 n$ componentes conexas, então $S$ é a união de $2 n$ subintervalos de $S^{1}$.

Prova. Como já mencionado anteriomente $\operatorname{tr} C_{z}$ é um polinômio de grau no máximo $2 n$, então $Q(z)=\frac{\operatorname{tr} C_{z}}{2 z^{n}}$ é uma função racional de grau no máximo $2 n$. Além disso,

$$
Q^{\prime}(z)=\frac{1}{2} \frac{\left(\operatorname{tr} C_{z}\right)^{\prime} z^{n}-n z^{n-1} \operatorname{tr} C_{z}}{z^{2 n}}=\frac{1}{2}\left(\frac{\left(\operatorname{tr} C_{z}\right)^{\prime}}{z^{n}}-n \frac{\operatorname{tr} C_{z}}{z^{n+1}}\right),
$$

e como $n \geq 1$ temos que $Q^{\prime}$ é também uma função racional de grau no máximo $2 n$. Para $z \in S^{1}$ temos $z=e^{i \theta}$ para algum $\theta \in \mathbb{R}$. Defina

$$
B_{\theta}:=\prod_{j=1}^{n} A_{j} R_{\theta}=A_{n} R_{\theta} \ldots A_{1} R_{\theta}
$$

Como

$$
C_{z}=\prod_{j=1}^{n} A_{j} \mathscr{R}(z)=\prod_{j=1}^{n} A_{j} z S_{z}
$$

temos $C_{e^{i \theta}}=\prod_{j=1}^{n} A_{j} e^{i \theta} S_{e^{i \theta}}=e^{i n \theta} \prod_{j=1}^{n} A_{j} R_{\theta}=e^{i n \theta} B_{\theta}$, e assim

$$
Q\left(e^{i \theta}\right)=\frac{\operatorname{tr} C_{e^{i \theta}}}{2 e^{i n \theta}}=\frac{e^{i n \theta} \operatorname{tr} B_{\theta}}{2 e^{i n \theta}}=\frac{\operatorname{tr} B_{\theta}}{2} \in \mathbb{R},
$$

pois $B_{\theta}$ é uma matriz real, já que $A_{j}$ e $R_{\theta}$ são matrizes reais, logo seu traço também é real e pela arbitrariedade de $z \in S^{1}$, segue que $Q\left(S^{1}\right) \subset \mathbb{R}$. Deste 
fato decorre que $Q^{\prime}$ tem ao menos um zero em cada componente conexa de $S^{1} \backslash S=S^{1} \backslash Q^{-1}([-1,1])$, fato este que provaremos a seguir. Vale ressaltar que estamos considerando a topologia de $S^{1}$, a qual é induzida de $\mathbb{R}^{2}$. Seja $I \subset S^{1} \backslash Q^{-1}([-1,1])$ uma componente conexa, queremos mostrar que $Q^{\prime}$ admite ao menos um zero em $I$. Considere $I=(a, b)$, o arco aberto com extremidades $a$ e $b$, que representa a menor distância entre $a$ e $b$, neste caso sendo o caminho que vai de $a$ à $b$ no sentido anti-horário. Já que $[-1,1]$ é fechado e $Q$ é, em particular, contínua, pois na realidade é holomorfa, temos $S=Q^{-1}([-1,1])$ fechado, consequentemente $S^{c}=S^{1} \backslash S$ é um aberto, e portanto $I \subset S^{1} \cap S^{c}$ é de fato um aberto, e ainda temos $a, b \in S$. Note que se $Q(a) \neq Q(b)$, podemos sem perda de generalidade assumir $Q(a)<Q(b)$, e então pelo Teorema do Valor Intermediário, para todo $d \in(Q(a), Q(b))$ existe $c \in(a, b)$ tal que $Q(c)=d \in(Q(a), Q(b)) \subset[-1,1]$, o que é uma contradição, pois $Q(I) \subset Q\left(S^{1} \cap S^{c}\right) \subset \mathbb{R} \backslash[-1,1]$. Portanto, temos $Q(a)=Q(b)$ e assim, pelo Teorema do Valor Médio existe $c \in I$ tal que

$$
Q^{\prime}(c)=\frac{Q(b)-Q(a)}{b-a}=0
$$

e então $Q^{\prime}$ admite ao menos um zero, neste caso $c$, em $I$. Bem, como $Q^{\prime}$ tem grau no máximo $2 n$ e possui ao menos um zero em cada componente conexa de $S^{1} \backslash S$, segue que $Q^{\prime}$ não pode ter zeros em $S$. Veremos a seguir que este fato, implica em cada componente conexa de $S \cap S^{1}$ ser mapeada difeomorficamente em $[-1,1]$. Seja agora $J \subset S^{1} \cap S$ uma componente conexa, perceba que $Q_{\uparrow_{J}}: J \rightarrow \mathbb{R}$ é injetiva, pois se existissem $c^{\prime}, d^{\prime} \in J$, com $c^{\prime} \neq d^{\prime}$ tais que $Q\left(c^{\prime}\right)=Q\left(d^{\prime}\right)$, pelo Teorema do Valor médio existiria $r \in\left(c^{\prime}, d^{\prime}\right)$, assumindo sem perda de generalidade o arco fechado $\left[c^{\prime}, d^{\prime}\right] \subset J$ análogamente ao que descrevemos antes, tal que

$$
Q^{\prime}(r)=\frac{Q\left(d^{\prime}\right)-Q\left(c^{\prime}\right)}{d^{\prime}-c^{\prime}}=0
$$

em que $r \in\left(c^{\prime}, d^{\prime}\right) \in J \subset S^{1} \cap S \Rightarrow r \in S$, o que seria uma contradição, pois como já discutimos, $Q^{\prime}$ não admite zeros em $S$. Mais ainda, restringindo o contra-domínio desta aplicação, tomando $Q_{\uparrow_{J}}: J \rightarrow Q(J)$, de modo a torná-la sobrejetiva e consequentemente bijetiva, e portanto invertível. Então $Q$ é diferenciável, invertível e não admite zeros em $J$, assim temos $Q^{-1}$ invertível e diferenciável. Temos ainda $J \subset S^{1} \cap S$ componente conexa, em que $S$ é fechado, então segue que $J$ é fechado, ou seja, é um arco fechado $J=[c, d] \subset S^{1}$, e já que $Q$ é contínua, $Q(J) \subset \mathbb{R}$ é conexo e fechado, isto é, é um intervalo fechado da reta. Portanto, basta compormos com um difeomorfismo que leve $Q(J)$ 
em $[-1,1]$, e sabemos que tal difeomorfismo existe, já que todos os intervalos fechados da reta são difeomorfos.

Em seguida, elucidaremos um lema que possui certo destaque, pois lida diretamente com a função $F$ definida sobre o espaço $\operatorname{PSL}(2, \mathbb{R}) \backslash\{[I]\}$, a qual temos trabalhado ao longo deste capítulo, assim como resultados obtidos no capítulo anterior, especialmente quando nos dedicamos a estudar os grupos fundamentais dos espaços $S^{1}, \operatorname{PSL}(2, \mathbb{R})$ e $\operatorname{PSL}(2, \mathbb{R}) \backslash\{[I]\}$. Como já utilizados anteriormente, estamos considerando os conjuntos

$$
X=\operatorname{PSL}(2, \mathbb{R}) \backslash\{[I]\}
$$

$\mathrm{e}$

$$
Y=\{[A] \in X:|\operatorname{tr} A| \leq 2\}
$$

Lema 4.14 Existe uma função contínua $F: X \rightarrow S^{1}$ tal que $F^{-1}(\{1\})=Y e$ o homomorfismo induzido $F_{\#}: \pi_{1}(X) \rightarrow \pi_{1}\left(S^{1}\right)$ é um isomorfismo.

Prova. Defina a função $F: X \rightarrow S^{1}$ dada por

$$
F([A])= \begin{cases}1, & \text { se }[A] \in Y \\ \frac{v^{2}}{w^{2}}, & \text { se }[A] \in X \backslash Y\end{cases}
$$

em que $v, w \in S^{1} \subset \mathbb{C}$ são autovetores de $A$ normalizados, com autodireções $\pm v, \pm w$ e autovalores associados $\lambda, \frac{1}{\lambda}$, respectivamente, com $|\lambda|>1$. Como vimos no Lema 4.10, F é uma função contínua, além disso é nítido que $F^{-1}(\{1\})=Y$. Considere a função $f:[-\pi, \pi] \rightarrow S^{1}$, dada por $f(\theta)=e^{i \theta}$ claramente contínua, a qual para uma matriz $M \in \mathrm{SL}(2, \mathbb{R}) \backslash\{I,-I\}$, simétrica, induz a função $\tilde{\gamma}: S^{1} \rightarrow \mathrm{SL}(2 \mathbb{R}) \backslash\{I,-I\}$ definida por $\tilde{\gamma}(f(\theta))=R_{\frac{\theta}{2}} M$, que também é contínua devido a continuidade de $f$. Defina agora a seguinte função, induzida por $\tilde{\gamma}$,

$$
\begin{aligned}
\gamma: S^{1} & \longrightarrow X \\
f(\theta) & \longmapsto\left[R_{\frac{\theta}{2}} M\right] .
\end{aligned}
$$

Tomaremos a liberdade de tratar $\gamma(f(\theta))$ por $\gamma(\theta)$ ou ainda $\gamma\left(e^{i \theta}\right)$. Perceba que para qualquer aberto $U \subset X$ temos para o aberto $A=\{M \in \mathrm{SL}(2, \mathbb{R}) \backslash\{I,-I\}:[M] \in U\}$

$$
\gamma^{-1}(U)=\{\theta \in[-\pi, \pi]: \gamma(\theta) \in U\}=\{\theta \in[-\pi, \pi]: \tilde{\gamma}(\theta) \in A\}=\tilde{\gamma}^{-1}(A),
$$


que é a pré-imagem de um aberto em $\operatorname{SL}(2, \mathbb{R}) \backslash\{I,-I\}$ por $\tilde{\gamma}$, deste modo, a continuidade de $\tilde{\gamma}$ implica a continuidade de $\gamma$. Além disso, $R_{\frac{\theta}{2}} M= \pm I$ se, e somente se $M= \pm\left(R_{\frac{\theta}{2}}\right)^{-1}= \pm R_{-\frac{\theta}{2}}$, e $R_{-\frac{\theta}{2}}$ é simétrica se, e somente se, $\theta=0$, que implicaria em termos $M= \pm I$, o que não é possível, então para todo $\theta \in[-\pi, \pi]$ temos $\gamma(f(\theta)) \in X$, mais ainda

$$
\gamma(f(-\pi))=\left[R_{\frac{-\pi}{2}} M\right]=\left[R_{\frac{\pi}{2}} M\right]=\gamma(f(\pi))
$$

portanto, $\gamma$ trata-se de um caminho fechado em $X$. Nosso próximo objetivo é mostrar que $F \circ \gamma$ é um caminho suave por partes, note que para $\theta \in[-\pi, \pi]$ e $M=\left(\begin{array}{ll}a & b \\ b & d\end{array}\right) \in \mathrm{SL}(2, \mathbb{R}) \backslash\{I,-I\}$ temos que

$$
\gamma(f(\theta))=\left[R_{\frac{\theta}{2}} M\right]=\left[\left(\begin{array}{ll}
a \cos \frac{\theta}{2}-b \sin \frac{\theta}{2} & b \sin \frac{\theta}{2}-d \sin \frac{\theta}{2} \\
a \sin \frac{\theta}{2}+b \cos \frac{\theta}{2} & b \sin \frac{\theta}{2}+d \cos \frac{\theta}{2}
\end{array}\right)\right]
$$

é tal que $\left|\operatorname{tr}\left(R_{\frac{\theta}{2}} M\right)\right|=2 \Longleftrightarrow(a+d) \cos \frac{\theta}{2}= \pm 2 \Longleftrightarrow \cos \frac{\theta}{2}= \pm \frac{2}{a+d}$, que tem um número finito de soluções em $[-\pi, \pi]$, as quais são as mesmas para $\left|\operatorname{tr}\left(-R_{\frac{\theta}{2}} M\right)\right|=2$. Então $F \circ \gamma: S^{1} \rightarrow S^{1}$, que pode também ser interpretada como $F \circ \gamma:[-\pi, \pi] \rightarrow S^{1}$, intersecta o subconjunto $\{[A] \in X:|\operatorname{tr} A|=2\}$ em um número finito de pontos. No Lema 4.10, vimos que para o conjunto $\{[A] \in[X]:|\operatorname{tr} A|<2\} \subset Y$ temos $F=1$, e para $[A] \in X \backslash Y$, sendo $v, w$ os autovetores de $A$, associados ao autovalor $\lambda, \frac{1}{\lambda}$ respectivamente, com $|\lambda|>1, \operatorname{temos} F([A])=\frac{\left(v^{2} /|v|^{2}\right)}{\left(w^{2} /|w|^{2}\right)}=\frac{v \bar{w}}{w \bar{v}}$ em que sempre tem-se $v \neq 0$ e $w \neq 0$, portanto fica clara a diferenciabilidade de $F$ nestes conjuntos. Contudo, como foi visto, tivemos mais trabalho para mostrar a continuidade de $F$ quando lidamos com o conjunto $\{[A] \in X:|\operatorname{tr} A|=2\}$, porém $F \circ \gamma$ intersecta este conjunto em um número finito de pontos, deste modo, podemos afirmar que $F$ é, ao menos, suave por partes, e em particular sabemos onde esta diferenciabilidade pode não ocorrer. Já que $F \circ \gamma$ é uma função suave por partes então faz sentido falarmos à respeito do índice desta função, perceba ainda que pelas propriedades $F\left(\left[A^{T}\right]\right)=\overline{F([A])}$ e $F\left(\left[R_{\theta}{ }^{-1} A R_{\theta}\right]\right)=F([A])$, provadas na Proposição 4.11, segue-se para $z=e^{i \theta} \in S^{1}$ que

$$
\begin{aligned}
\overline{F(\gamma(z))} & =\overline{F\left(\left[R_{\frac{\theta}{2}} M\right]\right)}=\overline{F\left(\left[\left(R_{\frac{\theta}{2}}^{-1}\right)^{-1} M R_{\frac{\theta}{2}}\left(R_{\frac{\theta}{2}}{ }^{-1}\right)\right]\right)} \\
& =\overline{F\left(\left[M R_{\frac{\theta}{2}}\right]\right)}=F\left(\left[\left(M R_{\frac{\theta}{2}}\right)^{T}\right]\right)=F\left(\left[R_{\frac{\theta}{2}}^{T} M^{T}\right]\right) \\
& =F\left(\left[R_{\frac{-\theta}{2}} M\right]\right)=F\left(\gamma\left(e^{-i \theta}\right)\right)=F(\gamma(\bar{z}))
\end{aligned}
$$

ou seja, $F \circ \gamma$ comuta com conjugação, de modo que $\overline{F(\gamma(z))}=F(\gamma(\bar{z}))$, a qual pode ser interpretada também como $\overline{F(\gamma(\theta))}=F(\gamma(-\theta))$ para $\theta \in[-\pi, \pi]$. 
Portanto, Tomando $\Gamma:=F \circ \gamma$ temos

$$
\begin{aligned}
\operatorname{ind}_{\Gamma}(0) & =\frac{1}{2 \pi i} \int_{-\pi}^{\pi} \frac{\Gamma^{\prime}(\theta)}{\Gamma(\theta)} d \theta=\frac{1}{2 \pi i}\left(\int_{-\pi}^{0} \frac{\Gamma^{\prime}(\theta)}{\Gamma(\theta)} d \theta+\int_{0}^{\pi} \frac{\Gamma^{\prime}(\theta)}{\Gamma(\theta)} d \theta\right) \\
& =\frac{1}{2 \pi i}\left(\int_{0}^{\pi} \frac{\Gamma^{\prime}(-\varphi)}{\Gamma(-\varphi)} d \varphi+\int_{0}^{\pi} \frac{\Gamma^{\prime}(\theta)}{\Gamma(\theta)} d \theta\right),
\end{aligned}
$$

tomando $\varphi=-\theta$. Já que $\Gamma(-\varphi)=\overline{\Gamma(\varphi)}$, temos $-\Gamma^{\prime}(-\varphi)=\overline{\Gamma^{\prime}(\varphi)}$, assim pela linearidade da integral

$$
\int_{0}^{\pi} \frac{\Gamma^{\prime}(-\varphi)}{\Gamma(-\varphi)} d \varphi=-\int_{0}^{\pi} \frac{\overline{\Gamma^{\prime}(\varphi)}}{\overline{\Gamma(\varphi)}} d \varphi=-\int_{0}^{\pi} \overline{\left(\frac{\Gamma^{\prime}(\varphi)}{\Gamma(\varphi)}\right)} d \varphi=-\overline{\int_{0}^{\pi} \frac{\Gamma^{\prime}(\varphi)}{\Gamma(\varphi)}} d \varphi
$$

Então, apenas trocando $\varphi$ por $\theta$ e denotando por $\operatorname{Im}(z)$ a parte imaginária do complexo $z$ temos

$$
\begin{aligned}
\operatorname{ind}_{\Gamma}(0) & =\frac{1}{2 \pi i}\left(\int_{0}^{\pi} \frac{\Gamma^{\prime}(\theta)}{\Gamma(\theta)}-\overline{\int_{0}^{\pi} \frac{\Gamma^{\prime}(\theta)}{\Gamma(\theta)}} d \theta\right)=\frac{1}{\pi} \operatorname{Im} \int_{0}^{\pi} \frac{\Gamma^{\prime}(\theta)}{\Gamma(\theta)} d \theta \\
& =\frac{1}{\pi} \int_{0}^{\pi} \operatorname{Im}\left(\frac{\Gamma^{\prime}(\theta)}{\Gamma(\theta)}\right) d \theta=\frac{1}{\pi} \int_{0}^{\pi}(\operatorname{Im} \log (\Gamma(\theta)))^{\prime} d \theta \\
& =\frac{1}{\pi} \int_{0}^{\pi}(\operatorname{Arg} \Gamma(\theta))^{\prime} d \theta=\frac{1}{\pi}(\operatorname{Arg}(\Gamma(\pi))-\operatorname{Arg}(\Gamma(0))) \\
& =\frac{1}{\pi}(\operatorname{Arg}(1)-\operatorname{Arg}(-1))=\frac{1}{\pi}(0-\pi)=-1,
\end{aligned}
$$

uma vez que

$$
\Gamma(\pi)=F(\gamma(\pi))=F\left(\left[R_{\frac{\pi}{2}} M\right]\right)=1,
$$

pelo fato de $\operatorname{tr} R_{\frac{\pi}{2}} M=0$, além disso, temos sempre $|\operatorname{tr} M|>2$ e como sabemos os autovalores de $M$ são ortogonais, isto é, são da forma $v= \pm i w$, assim $v^{2}=-w^{2}$ e portanto

$$
\Gamma(0)=F(\gamma(0))=F([M])=-1
$$

Logo, $\operatorname{grau}(F \circ \gamma)=\operatorname{ind}_{F \circ \gamma}(0)=-1$, desta maneira, como já discutimos na Seção 3.4, enquanto tratávamos das funções circulares, a classe de $F \circ \gamma$ representará $-1 \in \mathbb{Z} \cong \pi_{1}\left(S^{1}\right)$, que é um gerador do grupo cíclico $\mathbb{Z}$, e portanto, como $F$ induz um homomorfismo

$$
\begin{gathered}
F_{\#}: \pi_{1}(X) \cong \mathbb{Z} \longrightarrow \pi_{1}\left(S^{1}\right) \cong \mathbb{Z} \\
{[\gamma] \longmapsto[F \circ \gamma]}
\end{gathered}
$$

temos que $[\gamma]$ deve representar também um gerador em $\mathbb{Z} \cong \pi_{1}(X)$, e então, 
de fato trata-se de um isomorfismo.

Como já foi dito e pôde ser claramente observado, em grande parte dos resultados estamos trabalhando sobre o grupo $\mathrm{SL}(2, \mathbb{R})$, assim apresentaremos a seguir um conjunto denso em $\mathrm{SL}(2, \mathbb{R})$. Tal argumentação se torna interessante, pois em determinados casos a continuidade nos permite elucidar resultados sobre $\operatorname{SL}(2, \mathbb{R})$, provando o mesmo apenas para o referido conjunto denso.

Proposição 4.15 Para $n \in \mathbb{N}$ o conjunto

$$
X_{n}=\left\{\left(A_{1}, \ldots, A_{n}\right) \in S L(2, \mathbb{R})^{n}: B_{\theta}=\prod_{j=1}^{n} A_{j} R_{\theta} \neq \pm I \text { para todo } \theta \in[0,2 \pi]\right\}
$$

é denso em $S L(2, R)^{n}$.

Prova. É pertinente mencionarmos que apesar de fornecermos um argumento que provará o caso geral, faremos em particular a prova do caso $n=1$, para que possamos observar do que se trata, de fato, esta densidade, neste caso mais simples.

Tome $n=1$, então

$$
\begin{aligned}
X_{1} & =\left\{A_{1} \in \mathrm{SL}(2, \mathbb{R}): A_{1} \neq \pm R_{-\theta} \text { para todo } \theta \in[0,2 \pi]\right\} \\
& =\mathrm{SL}(2, \mathbb{R}) \backslash \mathrm{SO}(2, \mathbb{R}) .
\end{aligned}
$$

Fixe $A_{1} \in \mathrm{SL}(2, \mathbb{R})$ tal que para algum $\theta_{0} \in[0,2 \pi]$ tenhamos $A_{1} R_{\theta_{0}}= \pm I$, isto é, $A_{1}= \pm R_{-\theta_{0}} \notin \mathrm{SL}(2, \mathbb{R}) \backslash \mathrm{SO}(2, \mathbb{R})$. Defina,

$$
A_{1}^{\epsilon}:=\left(\begin{array}{cc}
e^{\epsilon} & 0 \\
0 & e^{-\epsilon}
\end{array}\right) A_{1} \in \mathrm{SL}(2, \mathbb{R}),
$$

a qual é tal que $\lim _{\epsilon \rightarrow 0} A_{1}^{\epsilon}=A_{1}$. O fato é que $A_{1}^{\epsilon} R_{\theta} \neq \pm I$ para todo $\theta \in[0,2 \pi]$ e para todo $\epsilon \in \mathbb{R}^{*}$, ou seja, temos $A_{1}^{\epsilon} \in \mathrm{SL}(2, \mathbb{R}) \backslash \mathrm{SO}(2, \mathbb{R})$. De fato, suponha que exista $\theta_{1} \in[0,2 \pi]$ tal que $A_{1}^{\epsilon} R_{\theta_{1}}= \pm I$, então

$$
\left(\begin{array}{cc}
e^{\epsilon} & 0 \\
0 & e^{-\epsilon}
\end{array}\right)= \pm I R_{-\theta_{1}} A_{1}^{-1}=\left( \pm R_{-\theta_{1}}\right)\left( \pm R_{\theta_{0}}\right)= \pm R_{\theta_{0}-\theta_{1}}
$$

em que $\pm R_{\theta_{0}-\theta_{1}}$ é uma matriz de rotação, porém $\left(\begin{array}{cc}e^{\epsilon} & 0 \\ 0 & e^{-\epsilon}\end{array}\right) \notin \mathrm{SO}(2, \mathbb{R})$ para todo $\epsilon \in R^{*}$, o que nos fornece uma contradição, portanto o caso $n=1$ está provado.

A seguir daremos um argumento geral que prova o caso em que $n \geq 1$, servindo 
ainda para o caso $n=1$. Fixe $A_{1}, \ldots, A_{n-1} \in \mathrm{SL}(2, \mathbb{R})$, se temos uma matriz $A_{n} \in \mathrm{SL}(2, \mathbb{R})$, tal que para algum $\theta_{0} \in[0,2 \pi]$ tem-se

$$
A_{n} R_{\theta_{0}} \ldots A_{1} R_{\theta_{0}}= \pm I
$$

então $\left(A_{1}, \ldots, A_{n}\right) \notin X_{n}$ e ainda $A_{n}= \pm R_{-\theta_{0}} A_{1}^{-1} \ldots R_{-\theta_{0}} A_{n-1}^{-1} R_{-\theta_{0}}$. Defina as seguintes curvas $\Phi_{+}$e $\Phi_{-}$, que na realidade tratam-se de caminhos fechados $\operatorname{em~} \operatorname{SL}(2, \mathbb{R})$,

$$
\begin{aligned}
\Phi_{ \pm}:[0,2 \pi] & \longrightarrow \operatorname{SL}(2, \mathbb{R}) \\
\theta & \longmapsto \pm R_{-\theta_{0}} A_{1}^{-1} \ldots R_{-\theta} A_{n-1}^{-1} R_{-\theta},
\end{aligned}
$$

as quais são claramente diferenciáveis, no sentido de que cada entrada de $\Phi_{ \pm}(\theta)$ é uma função diferenciável, para todo $\theta \in[0,2 \pi]$. Além disso, note que $A_{n}=\Phi_{+}\left(\theta_{0}\right)$ ou $A_{n}=\Phi_{-}\left(\theta_{0}\right)$, sendo $\Phi_{ \pm}$curvas contínuas em $\operatorname{SL}(2, \mathbb{R})$, que trata-se de uma variedade de dimensão 3 , então localmente é homeomorfa à uma bola em $\mathbb{R}^{3}$. Deste modo, em particular, localmente $A_{n} \in \Phi_{+}([0,2 \pi])$, ou $A_{n} \in \Phi_{-}([0,2 \pi])$, é levado por este homeomorfismo, para dentro de uma vizinhança contida em uma bola em $\mathbb{R}^{3}$, a qual possui dimensão 3 , e o fato da imagem curva $\Phi_{ \pm}$possuir dimensão menor que 3 , fica clara a existência de uma aproximação de $A_{n}, A_{n}^{\epsilon} \rightarrow A_{n}$, por matrizes $A_{n}^{\epsilon}=\left(\begin{array}{cc}e^{\epsilon} & 0 \\ 0 & e^{-\epsilon}\end{array}\right) A_{n} \in \mathrm{SL}(2, \mathbb{R})$ que não pertencem a curva $\Phi_{+}$, ou $\Phi_{-}$, no caso em que $A_{n}=\Phi_{+}\left(\theta_{0}\right)$, ou $A_{n}=\Phi_{+}\left(\theta_{0}\right)$, respectivamente. Portanto $A_{n}^{\epsilon} \neq \Phi_{+}(\theta)$, ou $A_{n}^{\epsilon} \neq \Phi_{-}(\theta)$, para todo $\theta \in[0,2 \pi]$ e para todo $\epsilon \in \mathbb{R}^{*}$, que implica em termos

$$
A_{n}^{\epsilon} R_{\theta} A_{n-1} R_{\theta} \ldots A_{1} R_{\theta} \neq I \quad \text { ou } \quad A_{n}^{\epsilon} R_{\theta} A_{n-1} R_{\theta} \ldots A_{1} R_{\theta} \neq-I,
$$

respectivamente, para todo $\theta \in[0,2 \pi]$ e para todo $\epsilon \in \mathbb{R}^{*}$, ou seja, $\left(A_{1}, \ldots, A_{n-1}, A_{n}^{\epsilon}\right) \in X_{n}$ para todo $\epsilon \in \mathbb{R}^{*}$, tal que para todo $\theta \in[0,2 \pi]$ $\lim _{\epsilon \rightarrow 0} A_{n}^{\epsilon} R_{\theta} A_{n-1} R_{\theta} \ldots A_{1} R_{\theta}=A_{n} R_{\theta} \ldots A_{1} R_{\theta}$, provando assim a densidade de $X_{n}$ em $\operatorname{SL}(2, \mathbb{R})^{n}$.

O lema a seguir nos fornecerá uma informação que diz respeito a uma propriedade topológica do conjunto $S \cap S^{1}$.

Lema 4.16 $S \cap S^{1}$ tem ao menos $2 n$ componentes conexas.

Prova. Considere a função $f:[-\pi, \pi] \rightarrow S^{1}$, dada por $f(\theta)=e^{i \theta}$, a qual induz a função $\tilde{g}: S^{1} \rightarrow \mathrm{SL}(2, \mathbb{R})$, dada por $g(f(\theta))=R_{\frac{\theta}{2}}$, que pode ser interpretada 
como um caminho em $\operatorname{SL}(2, \mathbb{R})$. Uma outra função, induzida por $\tilde{g}$ é

$$
\begin{aligned}
g: S^{1} & \longrightarrow \operatorname{PSL}(2, \mathbb{R}) \\
f(\theta) & \longmapsto\left[R_{\frac{\theta}{2}}\right]
\end{aligned}
$$

a qual também é contínua, pelo mesmo argumento apresentado no Lema anterior, de modo que a continuidade de $\tilde{g}$ implica na continuidade de $g$. Além disso, como

$$
g(f(\pi))=\left[R_{\frac{\pi}{2}}\right]=\left[-R_{\frac{\pi}{2}}\right]=\left[R_{\frac{-\pi}{2}}\right]=g(f(-\pi)),
$$

então $g$ trata-se de um caminho fechado em $\operatorname{PSL}(2, \mathbb{R})$, em que tomaremos a liberdade de nos referirmos a $g(f(\theta))$ apenas como $g(\theta)$ ou $g\left(e^{i \theta}\right)$. Queremos primeiramente mostrar que $g$ é um gerador do grupo fundamental de $\operatorname{PSL}(2, \mathbb{R})$. Como provamos na seção $3.4, \pi_{1}(\operatorname{PSL}(2, \mathbb{R})) \cong \mathbb{Z}$, então é suficiente provarmos que para qualquer $[\gamma] \in \pi_{1}(\operatorname{PSL}(2, \mathbb{R}))$ existe $m \in \mathbb{Z}$ tal que $[\gamma]=m[g]$. Seja $\gamma:[-\pi, \pi] \rightarrow \operatorname{PSL}(2, \mathbb{R})$ um caminho fechado em $\operatorname{SL}(2, \mathbb{R})$.

Como vimos na Decomposição de Iwasawa de $\operatorname{SL}(2, \mathbb{R})$, Teorema 3.56, para uma matriz $M=\left(\begin{array}{ll}a & b \\ c & d\end{array}\right) \in \mathrm{SL}(2, \mathbb{R})$ temos as seguinte funções contínuas

$$
\begin{aligned}
& r: \operatorname{SL}(2, \mathbb{R}) \longrightarrow(0,+\infty) \quad, \quad k: \operatorname{SL}(2, \mathbb{R}) \longrightarrow K \\
& M \longmapsto \sqrt{a^{2}+c^{2}} \quad M \longmapsto\left(\begin{array}{cc}
\frac{a}{r(M)} & -\frac{c}{r(M)} \\
\frac{c}{r(M)} & \frac{a}{r(M)}
\end{array}\right) \\
& a^{\prime}: \mathrm{SL}(2, \mathbb{R}) \longrightarrow A \quad \text { e } \quad n: \mathrm{SL}(2, \mathbb{R}) \longrightarrow N \\
& M \longmapsto\left(\begin{array}{cc}
r(M) & 0 \\
0 & \frac{1}{r(M)}
\end{array}\right) \quad M \longmapsto\left(\begin{array}{cc}
1 & \frac{a b+c d}{a^{2}+c^{2}} \\
0 & 1
\end{array}\right)
\end{aligned}
$$

em que $M=k(M) a^{\prime}(M) n(M)$.

Consequentemente $\operatorname{PSL}(2, \mathbb{R}) \ni[M]=\left\{k(M) a^{\prime}(M) n(M),-k(M) a^{\prime}(M) n(M)\right\}$ e perceba que, de fato, esta decomposição está bem definida para $\operatorname{PSL}(2, \mathbb{R})$ pois $r(M)=r(-M)$, que implica em $a^{\prime}(M)=a^{\prime}(-M)$, além disso, $n(M)=n(-M)$ e temos $k(M)$ identificado com

$$
-k(M)=\left(\begin{array}{cc}
-\frac{a}{r(M)} & \frac{c}{r(M)} \\
-\frac{c}{r(M)} & -\frac{a}{r(M)}
\end{array}\right)=\left(\begin{array}{cc}
-\frac{a}{r(-M)} & \frac{c}{r(-M)} \\
-\frac{c}{r(-M)} & -\frac{a}{r(-M)}
\end{array}\right)=k(-M) .
$$


Então, em particular para $\theta \in[-\pi, \pi]$ temos

$$
\gamma(\theta)=\left[k(\gamma(\theta)) a^{\prime}(\gamma(\theta)) n(\gamma(\theta))\right]
$$

em que como vimos, $\operatorname{PSL}(2, \mathbb{R}) \cong \mathrm{SO}(2, \mathbb{R}) / R_{\theta} \sim-R_{\theta} \times A \times N$ e portanto podemos interpretar $\gamma(\theta)$ como $\left([k(\gamma(\theta))], a^{\prime}(\gamma(\theta)), n(\gamma(\theta))\right)$. Note que $a^{\prime}$ é homotópica à $i_{A}: \mathrm{SL}(2, \mathbb{R}) \rightarrow A$, dada por $i_{A}(M)=I$, matriz identidade. De fato, defina a função

$$
\begin{aligned}
\tilde{A}: \operatorname{SL}(2, \mathbb{R}) \times[0,1] & \longrightarrow A \\
(M, t) & \longmapsto\left(\begin{array}{cc}
(1-t) r(M)+t & 0 \\
0 & \frac{1}{(1-t) r(M)+t}
\end{array}\right)
\end{aligned}
$$

a qual é contínua devido a continuidade de $r$, e o fato de termos sempre $r(M)>0$ implica $\tilde{A}(M, t) \in A$ para toda matriz $M \in \mathrm{SL}(2, \mathbb{R})$ e $t \in[0,1]$, mais ainda, temos $\tilde{A}(M, 0)=a^{\prime}(M)$ e $\tilde{A}(M, 1)=i_{A}(M)$. Note ainda que a função $n$ é homotópica à $i_{N}: \operatorname{SL}(2, \mathbb{R}) \rightarrow N$, dada por $i_{N}(M)=I$, como veremos. Defina a função

$$
\begin{aligned}
\tilde{N}: \operatorname{SL}(2, \mathbb{R}) \times[0,1] & \longrightarrow N \\
(M, t) & \longmapsto\left(\begin{array}{cc}
1 & (1-t) \frac{a b+c d}{a^{2}+c^{2}} \\
0 & 1
\end{array}\right)
\end{aligned}
$$

a qual é contínua pela continuidade de $n$, e como $(1-t) \frac{a b+c d}{a^{2}+c^{2}} \in \mathbb{R}$ para qualquer $t \in \mathbb{R}$ e $M=\left(\begin{array}{ll}a & b \\ c & d\end{array}\right) \in \mathrm{SL}(2, \mathbb{R})$ temos $\tilde{N}(M, t) \in \mathbb{N}$ para todo $t \in[0,1]$ e para toda $M \in \operatorname{SL}(2, \mathbb{R})$, mais ainda, temos $\tilde{N}(M, 0)=n(M)$ e $\tilde{N}(M, 1)=i_{N}(M)$. Sem perda de generalidade, afim de não carregarmos demasiadamente notações ou certo formalismo que não agregaria, podemos perceber que $\theta \mapsto\left[e^{i \frac{\theta}{2}}\right]$ é um gerador de $\pi_{1}\left(S^{1} / z \sim-z\right) \cong \pi_{1}(\operatorname{PSO}(2, \mathbb{R}))$, em que $\operatorname{PSO}(2, \mathbb{R}):=\operatorname{SO}(2, \mathbb{R}) /_{R_{\theta} \sim-R_{\theta}}$, e ainda pelos homeomorfismos discutidos na seção 3.4 do capítulo 3 , faz de $\theta \mapsto\left[R_{\frac{\theta}{2}}\right]$ um gerador de $\pi_{1}(\operatorname{PSO}(2, \mathbb{R})) \cong \mathbb{Z}$, portanto a classe de qualquer loop em $\pi_{1}(\operatorname{PSO}(2, \mathbb{R}))$ pode ser interpretada como um "múltiplo" de $[g]$, com respeito a operação definida para o grupo. Em particular para o caminho $k^{*}$, definido como $k^{*}(\theta)=[k(\gamma(\theta))] \in \operatorname{PSO}(2, \mathbb{R})$, existe $m \in \mathbb{Z}$ tal que $\left[k^{*}\right]=m[g]$. Contudo, como

$$
\begin{aligned}
\gamma & =\left[k(\gamma) a^{\prime}(\gamma) n(\gamma)\right] \cong\left([k(\gamma)], a^{\prime}(\gamma), n(\gamma)\right) \\
& \simeq\left([k(\gamma)], i_{A}(\gamma), i_{N}(\gamma)\right) \cong\left[k(\gamma) i_{A}(\gamma) i_{N}(\gamma)\right]=[k(\gamma)]=k^{*}
\end{aligned}
$$


temos $\gamma \simeq k^{*}$ e portanto $[\gamma]=\left[k^{*}\right]$, $\log 0[\gamma]=m[g]$. Pela arbitrariedade de $[\gamma] \in \pi_{1}(\operatorname{PSL}(2, \mathbb{R}))$, temos que $g$ é, de fato, um gerador de $\pi_{1}(\operatorname{PSL}(2, \mathbb{R}))$. Defina agora, para $n \in \mathbb{N}$ e $A_{1}, \ldots, A_{n} \in \mathrm{SL}(2, \mathbb{R})$ o caminho $\tilde{h}: S^{1} \longrightarrow \mathrm{SL}(2, \mathbb{R})$, definido por $f(\theta) \longmapsto B_{\theta}=\prod_{j=1}^{n} A_{j} R_{\theta}$, o qual, de forma análoga ao que discutimos, induz um caminho fechado

$$
\begin{aligned}
h: S^{1} & \longrightarrow \operatorname{PSL}(2, \mathbb{R}) \\
f(\theta) & \longmapsto\left[B_{\theta}\right] .
\end{aligned}
$$

Note que $\tilde{h} \simeq \tilde{g}^{2 n}=\tilde{g} \ldots \tilde{g}, 2 n$ vezes. De fato, para $n=1$ temos

$$
\tilde{g}^{2}\left(e^{i \theta}\right)=\tilde{g}\left(e^{i \theta}\right) \tilde{g}\left(e^{i \theta}\right)=R_{\frac{\theta}{2}} R_{\frac{\theta}{2}}=R_{\theta}
$$

Como vimos, $\mathrm{SL}(2, \mathbb{R})$ é conexo por caminhos, então existe $\alpha:[0,1] \rightarrow \operatorname{SL}(2, \mathbb{R})$ contínua tal que $\alpha(0)=A_{1}$ e $\alpha(1)=I$, assim podemos então definir a função

$$
\begin{aligned}
\tilde{\Gamma}:[0,1] \times S^{1} & \longrightarrow \operatorname{SL}(2, \mathbb{R}) \\
\left(t, e^{i \theta}\right) & \longmapsto \alpha(t) R_{\theta}
\end{aligned}
$$

a qual é contínua e tal que $\alpha(t) R_{\theta} \in \mathrm{SL}(2, \mathbb{R})$ para todo $t \in[0,1]$ e para todo $\theta$, e ainda é tal que

$$
\begin{aligned}
& \tilde{\Gamma}\left(0, e^{i \theta}\right)=\alpha(0) R_{\theta}=A_{1} R_{\theta}=B_{\theta}=\tilde{h}\left(e^{i \theta}\right), \\
& \tilde{\Gamma}\left(1, e^{i \theta}\right)=\alpha(1) R_{\theta}=I R_{\theta}=R_{\theta}=\tilde{g}^{2}\left(e^{i \theta}\right),
\end{aligned}
$$

e então temos, de fato, uma homotopia entre $\tilde{h}$ e $\tilde{g}^{2}$. Para o caso em que $n \geq 1$ temos os caminhos $\alpha_{j}:[0,1] \rightarrow \mathrm{SL}(2, \mathbb{R})$ contínuos com $\alpha_{j}(0)=A_{j}$ e $\alpha_{j}(1)=I$, e de modo análogo, sejam $\tilde{\Gamma}_{j}:[0,1] \times S^{1} \rightarrow \mathrm{SL}(2, \mathbb{R})$, definidas por $\tilde{\Gamma}_{j}\left(t, e^{i \theta}\right)=\alpha_{j}(t) R_{\theta}$ as homotopias entre $e^{i \theta} \mapsto \tilde{\Gamma}_{j}\left(0, e^{i \theta}\right)=A_{j} R_{\theta} \mathrm{e}$ $e^{i \theta} \mapsto \tilde{\Gamma}_{j}\left(1, e^{i \theta}\right)=I R_{\theta}=R_{\theta}=\tilde{g}^{2}\left(e^{i \theta}\right)$. Deste modo, $\tilde{\Gamma}\left(t, e^{i \theta}\right):=\prod_{j=1}^{n} \tilde{\Gamma}_{j}\left(t, e^{i \theta}\right)$ é uma homotopia entre $\tilde{h}$ e $\prod_{j=1}^{n} \tilde{g}^{2}=\tilde{g}^{2 n}$, a qual induz uma homotopia $\Gamma:[0,1] \times S^{1} \rightarrow \operatorname{PSL}(2, \mathbb{R})$, definida por $\Gamma\left(t, e^{i \theta}\right)=\left[\prod_{j=1}^{n} \tilde{\Gamma}\left(t, e^{i \theta}\right)\right]$, entre $h$ e $g^{2 n}$. Como vimos na proposição anterior, Proposição 4.15, podemos assumir $B_{\theta} \neq \pm I$ para todo $\theta \in[0,2 \pi]$, portanto podemos considerar $h\left(S^{1}\right) \subset \operatorname{PSL}(2, \mathbb{R}) \backslash\{[I]\}$, deste modo a composição $F \circ h$ faz sentido e está bem definida. Já que $\pi_{1}(\operatorname{PSL}(2, \mathbb{R})) \cong \pi_{1}(\operatorname{PSL}(2, \mathbb{R}) \backslash[I]) \cong \pi_{1}\left(S^{1}\right) \cong \mathbb{Z}$ e $h \simeq g^{2 n}$, que claramente possui grau $2 n$, então $h$ possui grau $2 n$ e, grosso modo, o homomorfismo induzido por $h$ pode ser interpretado como 


$$
\begin{aligned}
h_{\#}: \mathbb{Z} & \longrightarrow \mathbb{Z} \\
k & \longmapsto 2 n k .
\end{aligned}
$$

Além disso, pelo que foi provado no Lema $4.14, F$ induz, na realidade, um isomorfismo

$$
\begin{aligned}
F_{\#}: \mathbb{Z} & \longrightarrow \mathbb{Z} \\
k & \longmapsto-k .
\end{aligned}
$$

Portanto, podemos exibir o homomorfismo induzido por $F \circ h$ como sendo

$$
\begin{aligned}
(F \circ h)_{\#}: \mathbb{Z} & \longrightarrow \mathbb{Z} \longrightarrow \mathbb{Z} \\
k & \longmapsto 2 n k \longmapsto-2 n k,
\end{aligned}
$$

$\operatorname{logo} \operatorname{grau}(F \circ h)=-2 n$. Note que $1 \in(F \circ h)\left(S^{1}\right)$ por definição e pela afirmação a seguir, $(F \circ h)^{-1}(\{1\})$ possui ao menos $2 n$ componentes conexas.

Afirmação 4.17 Seja $g: S^{1} \rightarrow S^{1}$ uma função contínua, tal que $\operatorname{grau}(g)=m \in \mathbb{Z}$. Se $1 \in g\left(S^{1}\right)$, então $g^{-1}(\{1\})$ possui ao menos $|m|$ componentes conexas.

Prova. Seja $\tilde{g}: \mathbb{R} \rightarrow \mathbb{R}$ um levantamento de $g$, o qual sabemos que existe, de modo que $\tilde{g}$ é contínua e tal que $g \circ \pi=\pi \circ \tilde{g}$, com $\pi: \mathbb{R} \rightarrow S^{1}$ sendo a projeção natural, isto é, $\pi(\theta)=e^{2 \pi i \theta}$. Então, como definimos $m=\operatorname{grau}(g)=$ $\tilde{g}(x+1)-\tilde{g}(x)$, para todo $x \in \mathbb{R}$, em particular $\tilde{g}(1)=\tilde{g}(0)+m$. Tome $a:=\tilde{g}(0)$ e daí $\tilde{g}(1)=a+m$, portanto como $\tilde{g}$ é contínua, pela propriedade de Darboux $\tilde{g}([0,1))=[\tilde{g}(0), \tilde{g}(1))=[a, a+m)$, em que podemos escrever como a união disjunta

$$
\tilde{g}([0,1))=\sqcup_{j=1}^{m}[a+j-1, a+j) .
$$

Por hipótese $1 \in g\left(S^{1}\right)$, então existe $\theta_{0} \in[0,1)$ tal que $e^{2 \pi i \theta_{0}} \in S^{1}$ e

$$
1=g\left(e^{2 \pi i \theta_{0}}\right)=g\left(\pi\left(\theta_{0}\right)\right)=\pi\left(\tilde{g}\left(\theta_{0}\right)\right) .
$$

Perceba que para cada $j \in\{1, \ldots, m\}$ existe $\theta_{j} \in[a+j-1, a+j)$ tal que $\theta_{j}-\theta_{0} \in \mathbb{Z}$ e portanto $e^{2 \pi i \theta_{j}}=e^{2 \pi i \theta_{0}}$, consequentemente

$$
\pi\left(\tilde{g}\left(\theta_{j}\right)\right)=g\left(e^{2 \pi i \theta_{j}}\right)=g\left(e^{2 \pi i \theta_{0}}\right)=1 .
$$

Considere $\tilde{g}^{-1}\left(\left\{\theta_{j}\right\}\right)$ e tome $I_{j} \subset \tilde{g}^{-1}\left(\left\{\theta_{j}\right\}\right)$ um intervalo, ou seja, uma componente conexa, podendo ainda ser um único ponto. Como $\theta_{j} \in[a+$ 
$j-1, a+j) \subset[a, a+m)$ e $\tilde{g}([0,1))=[a, a+m)$, temos $I_{j} \subset[0,1)$ para $j \in\{1, \ldots, m\}$, além disso com $I_{1}, \ldots, I_{m}$ disjuntos dois a dois, pois $\theta_{j} \neq \theta_{i}$ para $j \neq i$. Seja $\tilde{I}_{j}:=\pi\left(I_{j}\right)$, pela continuidade de $\pi \tilde{I}_{j}$ é um conexo em $S^{1}$, e ainda temos $\tilde{I}_{1}, \ldots, \tilde{I_{m}}$ disjuntos dois a dois, em que

$$
g\left(\tilde{I}_{j}\right)=g\left(\pi\left(I_{j}\right)\right)=\pi\left(\tilde{g}\left(I_{j}\right)\right)=\pi\left(\theta_{j}\right)=1
$$

Portanto, para $j \in\{1, \ldots, m\} \tilde{I}_{j} \subset g^{-1}(\{1\})$ e por hipótese $g^{-1}(\{1\}) \subset S^{1}$, $\operatorname{logo} \tilde{I}_{j} \subset S^{1}$. Então $\tilde{I}_{1}, \ldots, \tilde{I_{m}}$ tratam-se de $m$ componentes conexas disjuntas de $g^{-1}(\{1\})$.

Note que por definição

$$
(F \circ h)^{-1}(\{1\})=\left\{e^{i \theta} \in S^{1}: F\left(\left[B_{\theta}\right]\right)=1\right\}=\left\{e^{i \theta} \in S^{1}:\left[B_{\theta}\right] \in Y\right\} \subset S^{1},
$$

além disso, lembre-se que temos $Q\left(e^{i \theta}\right)=\frac{\operatorname{tr} C_{e^{i \theta}}}{2 e^{i n \theta}}=\frac{\operatorname{tr}\left(e^{i n \theta} B_{\theta}\right)}{2 e^{i n \theta}}=\frac{1}{2} \operatorname{tr} B_{\theta}$, portanto

$$
e^{i \theta} \in S=Q^{-1}([-1,1]) \Longleftrightarrow\left|\operatorname{tr} B_{\theta}\right| \in[0,2] \Longleftrightarrow\left[B_{\theta}\right] \in Y,
$$

consequentemente temos $S \cap S^{1}=(F \circ h)^{-1}(\{1\})$ e então $S \cap S^{1}$ possui ao menos $2 n$ componentes conexas.

Finalmente estamos prontos para realizar a prova da Proposição 4.1, a qual evidencia a existência de uma parametrização holomorfa do espectro $\left\{\lambda_{1}(z), \lambda_{2}(z)\right\}$ da matriz $C_{z}$, para todo $z \in \mathbb{D}$, de modo que, $\left|\lambda_{2}(z)\right|<\left|\lambda_{1}(z)\right|$. Prova.[Prova da Proposição 4.1]

Note que temos

$$
\operatorname{det} \mathscr{R}(z)=\left(\frac{z^{2}+1}{2}\right)^{2}+\left(\frac{z^{2}-1}{2 i}\right)^{2}=\left(\frac{z^{2}+1}{2}\right)^{2}-\left(\frac{z^{2}-1}{2}\right)^{2}=z^{2}
$$

e como $C_{z}=\prod_{j=1}^{n} A_{j} \mathscr{R}(z)=A_{n} \mathscr{R}(z) \ldots A_{1} \mathscr{R}(z)$ temos

$$
\operatorname{det} C_{z}=\prod_{j=1}^{n} \operatorname{det} A_{j} \operatorname{det} \mathscr{R}(z)=\prod_{j=1}^{n} z^{2}=z^{2 n} .
$$

Lembre-se que no Lema 4.12 obtivemos um critério para identificar quando os autovalores de uma matriz complexa $2 \times 2$ possuem módulo igual, portanto, por este resultado, podemos afirmar que os autovalores de $C_{z}$ possuem mesmo 
módulo se, e somente se,

$$
\frac{\left(\operatorname{tr} C_{z}\right)^{2}}{4 \operatorname{det} C_{z}}=\frac{\left(\operatorname{tr} C_{z}\right)^{2}}{4 z^{2 n}} \in[0,1] \quad \Longleftrightarrow Q(z)=\frac{\operatorname{tr} C_{z}}{2 z^{n}} \in[-1,1]
$$

Para que possamos provar esta proposição, precisaremos primeiramente provar que para todo $z \in \mathbb{D}$ os autovalores de $C_{z}$ possuem módulo distinto, ou seja, que para todo $z \in \mathbb{D}$ tenhamos $Q(z) \notin[-1,1]$. A ideia é, já que $Q$ trata-se de uma função racional de grau no máximo $2 n$, o fato de $Q(z) \notin[-1,1]$ para todo $z \in \mathbb{D}$ pode ser provado mostrando que o circulo unitário contém todas as pré-imagens de $[-1,1]$. Como já definimos anteriormente $S=Q^{-1}([-1,1])$, lembre-se ainda que no Lema 4.16 provamos o fato de $S \cap S^{1}$ possuir ao menos $2 n$ componentes conexas, portanto pelo Lema 4.13 podemos afirmar que $S$ é a união de $2 n$ subintervalos de $S^{1}$, deste modo, temos $S \subset S^{1}$. Então, para todo $z \in \mathbb{C}$ tal que $Q(z) \in[-1,1]$, e consequentemente os autovalores de $C_{z}$ possuem mesmo módulo, devemos ter $z \in S^{1}=\partial \mathbb{D}$, o que nos permite afirmar, que para todo $z \in \mathbb{D}$ a matriz $C_{z}$ possui autovalores com módulo distinto. Considere por $\Lambda_{z}$ e $\lambda_{z}$ os autovalores de $C_{z}$, em que, como vimos, $\left|\Lambda_{z}\right| \neq\left|\lambda_{z}\right|$. Sem perda de generalidade, para todo $z \in \mathbb{D}$, tome $\Lambda_{z}$ o autovalor de $C_{z}$ que possui maior módulo, de modo que

$$
\left|\lambda_{z}\right|<\left|\Lambda_{z}\right|
$$

Nos resta então provar que as funções definidas a seguir são holomorfas

$$
\begin{aligned}
\lambda_{1}: \mathbb{D} & \longrightarrow \mathbb{C} \\
z & \longmapsto \lambda_{1}(z):=\Lambda_{z}, \\
\lambda_{2}: \mathbb{D} & \longrightarrow \mathbb{C} \\
z & \longmapsto \lambda_{2}(z):=\lambda_{z} .
\end{aligned}
$$

Como $\operatorname{det} C_{z}=\Lambda(z) \lambda(z)=\lambda_{1}(z) \lambda_{2}(z)$ e a função determinante é holomorfa, é suficiente provarmos que $\lambda_{1}$ é uma função holomorfa. Defina a função

$$
\begin{aligned}
f: \mathbb{C} & \longrightarrow \mathbb{C} \\
z & \longmapsto\left(\operatorname{tr} C_{z}\right)^{2}-4 \operatorname{det} C_{z},
\end{aligned}
$$

a qual é claramente holomorfa. Além disso, para todo $z \in \mathbb{D}$ temos $f(z) \neq 0$, pois caso existisse $z_{0} \in \mathbb{D}$ tal que $f\left(z_{0}\right)=0$, teríamos $\left(\operatorname{tr} C_{z_{0}}\right)^{2}=4 \operatorname{det} C_{z_{0}}$ e portanto $\frac{\left(\operatorname{tr} C_{0}\right)^{2}}{4 \operatorname{det} C_{z}}=1 \in[0,1]$, o que pelo Lema 4.12 implicaria em termos $\left|\lambda_{1}(z)\right|=\left|\lambda_{2}(z)\right|$, o que contradiria o que provamos. Como $f(z) \neq 0$ para 
todo $z \in \mathbb{D}$, sendo $\mathbb{D}$ simplesmente conexo, pela Observação 3.37 a função $\sqrt{f}=\exp \left(\frac{1}{2} \log f\right)$ possui um ramo holomorfo em $\mathbb{D}$. Como sabemos $\lambda_{1}(z), \lambda_{2}(z)$ são as raízes do polinômio característico de $C_{z}: \lambda^{2}-\lambda \operatorname{tr} C_{z}+\operatorname{det} C_{z}$ $\mathrm{com}$

$$
\lambda_{ \pm}=\frac{1}{2}\left(\operatorname{tr} C_{z} \pm \sqrt{\left(\operatorname{tr} C_{z}\right)-4 \operatorname{det} C_{z}}\right)=\frac{1}{2}\left(\operatorname{tr} C_{z} \pm \sqrt{f}\right),
$$

contudo não sabemos se $\lambda_{1}(z)=\lambda_{+}$ou $\lambda_{1}(z)=\lambda_{-}$. Defina então as funções

$$
\begin{aligned}
\varphi_{ \pm}: \mathbb{D} & \longrightarrow \mathbb{C} \\
z & \longmapsto \frac{1}{2}\left(\operatorname{tr} C_{z} \pm \sqrt{f}\right)
\end{aligned}
$$

em que $\left\{\varphi_{+}(z), \varphi_{-}(z)\right\}$ é o espectro de $C_{z}$ e pelo que discutimos $\varphi_{ \pm}$tratamse de funções holomorfas. Observe então que para concluirmos a Proposição em questão, é suficiente provarmos que localmente $\lambda_{1}(z)=\varphi_{+}(z)$ ou $\lambda_{1}(z)=\varphi_{-}(z)$, uma vez que a holomorfia é uma propriedade local. Assim, fixe $z_{0} \in \mathbb{D}$. Por definição $\lambda_{1}\left(z_{0}\right)=\varphi_{+}\left(z_{0}\right)$ ou $\lambda_{1}\left(z_{0}\right)=\varphi_{-}\left(z_{0}\right)$. Sem perda de generalidade assuma $\lambda_{1}\left(z_{0}\right)=\varphi_{+}\left(z_{0}\right)$, então consequentemente $\lambda_{2}\left(z_{0}\right)=\varphi_{-}\left(z_{0}\right)$. Como $\left|\lambda_{1}\left(z_{0}\right)\right|>\left|\lambda_{2}\left(z_{0}\right)\right|$ temos $\left|\varphi_{-}\left(z_{0}\right)\right|<\left|\varphi_{+}\left(z_{0}\right)\right|$, em que as funções $z \mapsto\left|\varphi_{ \pm}(z)\right|$ são contínuas, portanto existe $r>0$ tal que $\left|\varphi_{-}(z)\right|<\left|\varphi_{+}(z)\right|$, para todo $z \in D\left(z_{0}, r\right)$. Logo na vizinhança $D\left(z_{0}, r\right)$ temos

$$
\lambda_{1}(z)=\varphi_{+}(z) \quad \text { e } \quad \lambda_{2}(z)=\varphi_{-}(z)
$$

portanto $\lambda_{1}$ é holomorfa em $D\left(z_{0}, r\right)$ e em particular em $z_{0}$. Como tomamos $z_{0} \in \mathbb{D}$ arbitrário, segue que $\lambda_{1}$ é holomorfa em $\mathbb{D}$ e assim $\lambda_{2}$ também é holomorfa em $\mathbb{D}$.

O próximo lema a ser evidenciado é bastante simples e tem por objetivo explicitar o raio espectral da matriz $C_{0}$.

Lema 4.18 A matriz $C_{0}=\prod_{j=1}^{n} A_{j} \mathscr{R}(0)$ tem raio espectral

$$
\rho\left(C_{0}\right)=\prod_{j=1}^{n} \frac{1}{2}\left(\left\|A_{j}\right\|+\left\|A_{j}\right\|^{-1}\right) .
$$

Prova. Primeiramente verificaremos três fatos importantes, para que possamos concluir a prova.

1. Podemos encontrar $\alpha_{j}, \beta_{j} \in[0,2 \pi]$ e $c_{j} \geq 1$ tais que $A_{j}=R_{\beta_{j}} H_{c_{j}} R_{\alpha_{j}}$ para $j \in\{1, \cdots, n\}$. Além disso, $\left\|A_{j}\right\|=c_{j}$.

Pelo Teorema da Decomposição em Valores Singulares, e as observações $4.4,4.5$ e 4.6 , fica clara a existência de tais $\alpha_{j}, \beta_{j} \in[0,2 \pi]$ e $c_{j} \geq 1$. 
2. Sejam $A, B \in \mathrm{SL}(2, \mathbb{R})$, temos $\rho(A B)=\rho(B A)$.

Sabemos que $|\lambda I-A B|=\lambda^{2}-\lambda \operatorname{tr} A B+1$ e analogamente $|\eta I-B A|=\eta^{2}-\eta \operatorname{tr} B A+1$. Portanto, neste caso, os autovalores de $A B$ e $B A$ dependem única e exclusivamente do traço de $A B$ e do traço de $B A$, respecivamente. Contudo, sabemos que $\operatorname{tr} A B=\operatorname{tr} B A$, assim segue que os autovalores de $A B$ e $B A$ são iguais, consequentemente $\rho(A B)=\rho(B A)$.

3. Para todo $\theta \in \mathbb{R}, R_{\theta} \mathscr{R}(0)=\mathscr{R}(0) R_{\theta}=e^{-i \theta} \mathscr{R}(0)$.

Sejam $z, w \in \mathbb{C}^{*}$, para

$$
\left(\begin{array}{ll}
a_{11} & a_{12} \\
a_{21} & a_{22}
\end{array}\right):=S_{z} S_{w}=\left(\begin{array}{cc}
\frac{z+z^{-1}}{2} & -\frac{z-z^{-1}}{2 i} \\
\frac{z-z^{-1}}{2 i} & \frac{z+z^{-1}}{2}
\end{array}\right)\left(\begin{array}{cc}
\frac{w+w^{-1}}{2} & -\frac{w-w^{-1}}{2 i} \\
\frac{w-w^{-1}}{2 i} & \frac{w+w^{-1}}{2}
\end{array}\right)
$$

temos

$$
\begin{aligned}
& a_{11}=\left(\frac{z+z^{-1}}{2}\right)\left(\frac{w+w^{-1}}{2}\right)-\left(\frac{z-z^{-1}}{2 i}\right)\left(\frac{w-w^{-1}}{2 i}\right)=\frac{z w+(z w)^{-1}}{2} \\
& a_{12}=\left(\frac{z+z^{-1}}{2}\right)\left(\frac{w-w^{-1}}{2 i}\right)-\left(\frac{z-z^{-1}}{2 i}\right)\left(\frac{w+w^{-1}}{2}\right)=-\frac{z w-(z w)^{-1}}{2 i} \\
& a_{21}=\left(\frac{z-z^{-1}}{2 i}\right)\left(\frac{w+w^{-1}}{2}\right)+\left(\frac{z+z^{-1}}{2}\right)\left(\frac{w-w^{-1}}{2 i}\right)=\frac{z w-(z w)^{-1}}{2 i} \\
& a_{22}=\left(\frac{z-z^{-1}}{2 i}\right)\left(\frac{w-w^{-1}}{2 i}\right)+\left(\frac{z+z^{-1}}{2}\right)\left(\frac{w+w^{-1}}{2}\right)=\frac{z w+(z w)^{-1}}{2}
\end{aligned}
$$

Portanto, $S_{z} S_{w}=S_{z w}$ e já que $\mathscr{R}(z)=z S_{z}$, segue que

$$
\mathscr{R}(z w)=z w S_{z w}=z w S_{z} S_{w}=z S_{z} w S_{w}=\mathscr{R}(z) \mathscr{R}(w)
$$

além disso, como $R_{\theta}=S_{e^{i \theta}}$ temos

$$
\begin{aligned}
R_{\theta} \mathscr{R}(0) & =S_{e^{i \theta}} \mathscr{R}(0)=e^{-i \theta} \mathscr{R}\left(e^{i \theta}\right) \mathscr{R}(0)=e^{-i \theta} \mathscr{R}(0) \mathscr{R}\left(e^{i \theta}\right) \\
& =\mathscr{R}(0) e^{-i \theta} \mathscr{R}\left(e^{i \theta}\right)=\mathscr{R}(0) R_{\theta} .
\end{aligned}
$$

Por outro lado,

$$
R_{\theta} \mathscr{R}(0)=e^{-i \theta} \mathscr{R}\left(e^{i \theta}\right) \mathscr{R}(0)=e^{-i \theta} \mathscr{R}(0) .
$$

Deste modo, utilizando os itens provados acima 1, 2 e 3 obtemos

$$
\rho\left(C_{0}\right)=\rho\left(\prod_{j=1}^{n} A_{j} \mathscr{R}(0)\right)=\rho\left(\prod_{j=1}^{n} R_{\beta_{j}} H_{c_{j}} R_{\alpha_{j}} \mathscr{R}(0)\right)=\rho\left(\prod_{j=1}^{n} R_{\beta_{j}} H_{c_{j}} e^{-i \alpha_{j}} \mathscr{R}(0)\right)
$$




$$
\begin{aligned}
& =\rho\left(\prod_{j=1}^{n} e^{i\left(-\alpha_{j}\right)} \prod_{j=1}^{n} R_{\beta_{j}} H_{c_{j}} \mathscr{R}(0)\right)=\rho\left(\prod_{j=1}^{n} R_{\beta_{j}} H_{c_{j}} \mathscr{R}(0)\right) \\
& =\rho\left(\prod_{j=1}^{n}\left(R_{\beta_{j}} H_{c_{j}}\right) \mathscr{R}(0)\right)=\rho\left(\prod_{j=1}^{n} \mathscr{R}(0)\left(R_{\beta_{j}} H_{c_{j}}\right)\right)=\rho\left(\prod_{j=1}^{n} e^{\left.-i \beta_{j} \mathscr{R}(0) H_{c_{j}}\right)}\right. \\
& =\rho\left(\prod_{j=1}^{n} \mathscr{R}(0) H_{c_{j}}\right) .
\end{aligned}
$$

Note que, como $\mathscr{R}(0)=\left(\begin{array}{cc}\frac{1}{2} & \frac{1}{2 i} \\ -\frac{1}{2 i} & \frac{1}{2}\end{array}\right)$ e $H_{c_{j}}=\left(\begin{array}{cc}c_{j} & 0 \\ 0 & c_{j}{ }^{-1}\end{array}\right)$ temos $\mathscr{R}(0) H_{c_{j}}=\left(\begin{array}{cc}\frac{c_{j}}{2} & \frac{c_{j}{ }^{-1}}{2 i} \\ -\frac{c_{j}}{2 i} & \frac{c_{j}{ }^{-1}}{2}\end{array}\right)$, e ainda

$$
\mathscr{R}(0) H_{c_{j}}\left(\begin{array}{c}
-i \\
1
\end{array}\right)=\left(\begin{array}{c}
-\frac{i c_{j}}{2}+\frac{c_{j}^{-1}}{2 i} \\
\frac{c_{j}}{2}+\frac{c_{j}^{-1}}{2}
\end{array}\right)=\left(\begin{array}{c}
\frac{-i\left(c_{j}+c_{j}-1\right.}{2} \\
\frac{c_{j}+c_{j}-1}{2}
\end{array}\right)=\frac{1}{2}\left(c_{j}+c_{j}^{-1}\right)\left(\begin{array}{c}
-i \\
1
\end{array}\right),
$$

para todo $j \in\{1, \ldots, n\}$. Portanto, cada matriz $\mathscr{R}(0) H_{c_{j}}$ possui $(-i, 1)$ como um autovetor, o qual é associado ao autovalor $\frac{1}{2}\left(c_{j}+c_{j}{ }^{-1}\right)$, para todo $j \in\{1, \ldots, n\}$. Consequentemente temos

$$
\begin{aligned}
\prod_{j=1}^{n} \mathscr{R}(0) H_{c_{j}}(-i, 1) & =\left(\mathscr{R}(0) H_{c_{n}} \ldots \mathscr{R}(0) H_{c_{1}}\right)(-i, 1) \\
& =\left(\mathscr{R}(0) H_{c_{n}} \ldots \mathscr{R}(0) H_{c_{2}}\right)\left(\mathscr{R}(0) H_{c_{1}}(-i, 1)\right) \\
& =\left(\mathscr{R}(0) H_{c_{n}} \ldots \mathscr{R}(0) H_{c_{2}}\right) \frac{1}{2}\left(c_{1}+c_{1}^{-1}\right)(-i, 1) \\
& =\frac{1}{2}\left(c_{1}+c_{1}^{-1}\right)\left(\mathscr{R}(0) H_{c_{n}} \ldots \mathscr{R}(0) H_{c_{3}}\right)\left(\mathscr{R}(0) H_{c_{2}}(-i, 1)\right) \\
& =\cdots=\prod_{j=1}^{n} \frac{1}{2}\left(c_{j}+c_{j}^{-1}\right)
\end{aligned}
$$

isto é, $\prod_{j=1}^{n} \mathscr{R}(0) H_{c_{j}}$ tem $(-i, 1)$ como um autovetor, o qual é associado ao autovalor $\prod_{j=1}^{n} \frac{1}{2}\left(c_{j}+c_{j}^{-1}\right)$. Como $\operatorname{det}\left(\prod_{j=1}^{n} \mathscr{R}(0) H_{c_{j}}\right)=0$. Então,

$$
\begin{aligned}
0 & =\left|\lambda I-\left(\prod_{j=1}^{n} \mathscr{R}(0) H_{c_{j}}\right)\right|=\lambda^{2}-\lambda \operatorname{tr}\left(\prod_{j=1}^{n} \mathscr{R}(0) H_{c_{j}}\right) \\
& \Rightarrow \lambda_{1}=0 \quad \text { e } \quad \lambda_{2}=\operatorname{tr}\left(\prod_{j=1}^{n} \mathscr{R}(0) H_{c_{j}}\right)=\prod_{j=1}^{n} \frac{1}{2}\left(c_{j}+c_{j}^{-1}\right),
\end{aligned}
$$

em que $\lambda_{1}$ e $\lambda_{2}$ são os autovalores de $\prod_{j=1}^{n} \mathscr{R}(0) H_{c_{j}}$. Assim sendo, já que $\left\|A_{j}\right\|=c_{j}>0$ temos $\rho\left(C_{0}\right)=\prod_{j=1}^{n} \frac{1}{2}\left(c_{j}+c_{j}{ }^{-1}\right)=\prod_{j=1}^{n} \frac{1}{2}\left(\left\|A_{j}\right\|+\left\|A_{j}\right\|^{-1}\right)$. 
Ao ponto em que estamos, munidos de todos os resultados apresentados no decorrer do capítulo, nos encontramos prontos a realizar a prova do teorema que será fundamental na demonstração da Fórmula de Avila-Bochi-Herman.

Teorema 4.19 Sejam $A_{1}, \ldots, A_{n} \in S L(2, \mathbb{R})$. Então

$$
\frac{1}{2 \pi} \int_{0}^{2 \pi} \log \rho\left(A_{n} R_{\theta} \ldots A_{1} R_{\theta}\right) d \theta=\sum_{j=1}^{n} N\left(A_{j}\right)
$$

Prova. A prova deste teorema é baseada nos métodos de complexificação exibidos na Desigualdade de Herman, Teorema 2.2, apresentado no capítulo 2. Para $n \in \mathbb{N}$, a continuidade da função

$$
\begin{aligned}
f: \operatorname{SL}(2, \mathbb{R})^{n} \times[0,2 \pi] & \longrightarrow \mathbb{R} \\
\left(A_{1}, \ldots, A_{n}, \theta\right) & \longmapsto \int_{0}^{2 \pi} \log \rho\left(A_{n} R_{\theta} \ldots A_{1} R_{\theta}\right) d \theta
\end{aligned}
$$

a qual foi provada no Lema 4.9, nos permite provar o resultado em questão apenas para um conjunto denso em $\operatorname{SL}(2, \mathbb{R})$. No Lema 4.15 , provamos que o conjunto

$$
X_{n}\left\{\left(A_{1}, \ldots, A_{n}\right) \in \mathrm{SL}(2, \mathbb{R})^{n}: B_{\theta}=\prod_{j=1}^{n} A_{j} R_{\theta} \neq \pm I \text { para todo } \theta \in[0,2 \pi]\right\}
$$

é denso em $\mathrm{SL}(2, \mathbb{R})^{n}$, então é suficiente provarmos o resultado para para matrizes $A_{1}, \ldots, A_{n} \in \mathrm{SL}(2, \mathbb{R})$ tais que $B_{\theta}=\prod_{j=1}^{n} A_{j} R_{\theta} \neq \pm I$ para todo $\theta \in[0,2 \pi]$. Lembre-se das matrizes complexas que definimos

$$
S_{z}=\left(\begin{array}{cc}
\frac{z+z^{-1}}{2} & -\frac{z-z^{-1}}{2 i} \\
\frac{z-z^{-1}}{2 i} & \frac{z+z^{-1}}{2}
\end{array}\right) \quad \text { para } z \in \mathbb{C}^{*}
$$

$\mathrm{e}$

$$
\mathscr{R}(z)=\left(\begin{array}{cc}
\frac{z^{2}+1}{2} & -\frac{z^{2}-1}{2 i} \\
\frac{z^{2}-1}{2 i} & \frac{z^{2}+1}{2} .
\end{array}\right) \quad \text { para } z \in \mathbb{C},
$$

em que $\mathscr{R}(z)=z S_{z}$, além disso, como $\cos \theta=\frac{e^{i \theta}+e^{-i \theta}}{2}$ e $\sin \theta=\frac{e^{i \theta}-e^{-i \theta}}{2 i}$, fica claro que temos

$$
S_{e^{i \theta}}=\left(\begin{array}{cc}
\cos \theta & -\sin \theta \\
\sin \theta & \cos \theta
\end{array}\right) \quad \text { para } \theta \in \mathbb{R}
$$

Então, estamos complexificando a matriz de rotação por meio da matriz $S_{z}$, em que obtemos a igualdade no caso em que $e^{i \theta}$. No entando, como pudemos 
observar, tal complexificação nos gerou uma singularidade em $z=0$, portanto, afim de lidarmos com essa singularidade, definimos $\mathscr{R}(z)=z S_{z}$, contudo, veremos a frente que esta tentativa de lidar com a singularidade nos gerará um certo "fator" $z^{n}$. Lembre-se ainda que para $A_{1}, \ldots, A_{n} \in \mathrm{SL}(2, \mathbb{R})$ definimos

$$
C_{z}=\prod_{j=1}^{n} A_{j} \mathscr{R}(z)=A_{n} \mathscr{R}(z) \ldots A_{1} \mathscr{R}(z) \quad \text { para } z \in \mathbb{C}
$$

que de certo modo, representa um iterado complexificado de $A_{n} R_{\theta} \ldots A_{1} R_{\theta}$. Pela Proposição 4.1, para todo $z \in \mathbb{D}$, sendo $\lambda_{1}(z), \lambda_{2}(z)$ os autovalores da matriz $C_{z}$, temos $0 \leq\left|\lambda_{2}(z)\right|<\left|\lambda_{1}(z)\right|$, portanto $\rho\left(C_{z}\right)=\left|\lambda_{1}(z)\right|>0$, e temos ainda $\mathbb{D}$ um domínio aberto e simplesmente conexo, $\lambda_{1}: \mathbb{D} \rightarrow \mathbb{C}$ uma função holomorfa, a qual não possui zeros em $\mathbb{D}$, portanto $\log \circ \lambda_{1}$ é um ramo holomorfo do logaritmo complexo $\log \circ \lambda_{1}$, em $\mathbb{D}$. Deste modo

$$
\log \left(\lambda_{1}(z)\right)=\log \left|\lambda_{1}(z)\right|+i \operatorname{Arg}\left(\lambda_{1}(z)\right)
$$

é holomorfa, e como sabemos a parte real de uma função holomorfa é harmônica, o que faz de $\log$ o| $\lambda_{1} \mid$ uma função harmônica em $\mathbb{D}$. Por outro lado, na Proposição 4.8, provamos que o raio espectral $\operatorname{Mat}(2, \mathbb{C}) \ni M \mapsto \rho(M)$ é uma função contínua, e claramente a função $\overline{\mathbb{D}} \ni z \mapsto C_{z} \in \operatorname{Mat}(2, \mathbb{C})$ é contínua, portanto a função

$$
\overline{\mathbb{D}} \ni z \longmapsto \log \rho\left(C_{z}\right)
$$

é contínua, além de ser harmônica em $\mathbb{D}$. Note ainda que tomando $z=e^{i \theta} \in \partial \mathbb{D}$ temos

$$
C_{z}=\prod_{j=1}^{n} A_{j} \mathscr{R}(z)=\prod_{j=1}^{n} A_{j} z S_{z}=z^{n} \prod_{j=1}^{n} A_{j} R_{\theta}=z^{n} B_{\theta},
$$

e podemos observar o fator $z^{n}$ obtido durante o processo de complexificação. Contudo perceba que este fator não influenciará no resultado que queremos obter, já que para $z=e^{i \theta}$ temos $\rho\left(C_{z}\right)=\rho\left(B_{\theta}\right)$, pois $\rho\left(B_{\theta}\right)=\rho\left(z^{n} B_{\theta}\right)$. De fato, seja $v \in \mathbb{R}^{2}$ um autovetor de $B_{\theta}$ associado à um autovalor $\alpha \in \mathbb{R}$, então $B_{\theta} v=\alpha v$ e consequentemente $z^{n}\left(B_{\theta} v\right)=z^{n} \alpha v$, portanto $z^{n} \alpha$ é um autovalor de $z^{n} B_{\theta}$, associado ao autovetor $v$, e temos $|\alpha|=\left|z^{n}\right||\alpha|=\left|z^{n} \alpha\right|$, já que $z=e^{i \theta}$, portanto, pela arbitrariedade do autovalor $\alpha$, os raios espectrais coincidem. Pela caracterização de funções harmônicas, chamada também como a propriedade do valor médio para funções harmônicas, Corolário 3.26, e ainda pelos fatos relatados, segue que

$$
\log \rho\left(C_{0}\right)=\log \left(\left|\lambda_{1}(0)\right|\right)=\frac{1}{2 \pi} \int_{0}^{2 \pi} \log \left(\lambda_{1}\left(e^{i \theta}\right)\right) d \theta
$$




$$
\begin{aligned}
& =\frac{1}{2 \pi} \int_{0}^{2 \pi} \log \rho\left(C_{e^{i \theta}}\right) d \theta=\frac{1}{2 \pi} \int_{0}^{2 \pi} \log \rho\left(B_{\theta}\right) d \theta \\
& =\frac{1}{2 \pi} \int_{0}^{2 \pi} \log \rho\left(A_{n} R_{\theta} \ldots A_{1} R_{\theta}\right) d \theta
\end{aligned}
$$

Por fim, pelo Lema 4.18, temos

$$
\rho\left(C_{0}\right)=\prod_{j=1}^{n} \frac{1}{2}\left(\left\|A_{j}\right\|+\left\|A_{j}\right\|^{-1}\right),
$$

então

$$
\begin{aligned}
\log \rho\left(C_{0}\right) & =\log \left(\prod_{j=1}^{n} \frac{1}{2}\left(\left\|A_{j}\right\|+\left\|A_{j}\right\|^{-1}\right)\right) \\
& =\sum_{j=1}^{n} \log \left(\frac{\left\|A_{j}\right\|+\left\|A_{j}\right\|^{-1}}{2}\right)=\sum_{j=1}^{n} N\left(A_{j}\right) .
\end{aligned}
$$

Logo, por 4-4 e 4-5 temos

$$
\frac{1}{2 \pi} \int_{0}^{2 \pi} \log \rho\left(A_{n} R_{\theta} \ldots A_{1} R_{\theta}\right) d \theta=\sum_{j=1}^{n} N\left(A_{j}\right) .
$$

Um corolário imediato do Teorema provado acima, o qual inclusive tem uma relação interessante com o mesmo, será provado a seguir.

Corolário 4.20 Sejam $A_{1}, \ldots, A_{n} \in S L(2, \mathbb{R})$. Então

$$
\frac{1}{2 \pi} \int_{0}^{2 \pi} N\left(A_{n} R_{\theta} \cdots A_{1} R_{\theta}\right) d \theta=\sum_{j=1}^{n} N\left(A_{j}\right)
$$

Prova. Sejam $A_{1}, \cdots, A_{n} \in \mathrm{SL}(2, R)$. Para $\theta \in[0,2 \pi]$, considere $B_{\theta}=\prod_{j=1}^{n} A_{j} R_{\theta}=A_{n} R_{\theta} \cdots A_{1} R_{\theta}$. Então, fixando $\theta \in[0,2 \pi]$ temos $B_{\theta} \in \mathrm{SL}(2, R)$, pois $\operatorname{det} B_{\theta}=\operatorname{det} A_{n} \operatorname{det} R_{\theta} \cdots \operatorname{det} A_{1} \operatorname{det} R_{\theta}=1$. Fixando $\theta^{\prime} \in[0,2 \pi]$, segue que

$$
\begin{aligned}
\frac{1}{2 \pi} \int_{0}^{2 \pi} \log \rho\left(B_{\theta} R_{\theta^{\prime}}\right) d \theta & =\frac{1}{2 \pi} \int_{0}^{2 \pi} \log \rho\left(A_{n} R_{\theta} \cdots A_{1} R_{\theta} R_{\theta^{\prime}}\right) d \theta \\
& =\frac{1}{2 \pi} \int_{0}^{2 \pi} \log \rho\left(A_{n} R_{\theta} \cdots A_{1} R_{\theta^{\prime}} R_{\theta}\right) d \theta \\
& =N\left(A_{1} R_{\theta^{\prime}}\right)+\sum_{j=2}^{n} N\left(A_{j}\right) \\
& =\sum_{j=1}^{n} N\left(A_{j}\right)
\end{aligned}
$$


em que, (4-6) se dá pelo fato de que, para quaisquer $\theta, \beta \in[0,2 \pi]$, as matrizes de rotação $R_{\theta}$ e $R_{\beta}$ comutam, ou seja, $R_{\theta} R_{\beta}=R_{\beta} R_{\theta}$. (4-7) segue como aplicação direta do Teorema 4.19. Já o item (4-8) é consequência de termos $\|A\|=\left\|A R_{\theta}\right\|$ e assim

$$
N\left(A R_{\theta}\right)=\log \left(\frac{\left\|A R_{\theta}\right\|+\left\|A R_{\theta}\right\|^{-1}}{2}\right)=\log \left(\frac{\|A\|+\|A\|^{-1}}{2}\right)=N(A)
$$

Novamente pelo Teorema 4.19 temos

$$
\frac{1}{2 \pi} \int_{0}^{2 \pi} \log \rho\left(B_{\theta} R_{\theta^{\prime}}\right) d \theta^{\prime}=N\left(B_{\theta}\right)
$$

Deste modo,

$$
\begin{aligned}
\frac{1}{2 \pi} \int_{0}^{2 \pi} N\left(B_{\theta}\right) d \theta & =\frac{1}{2 \pi} \int_{0}^{2 \pi} \frac{1}{2 \pi} \int_{0}^{2 \pi} \log \rho\left(B_{\theta} R_{\theta^{\prime}}\right) d \theta d \theta^{\prime} \\
& =\frac{1}{2 \pi} \int_{0}^{2 \pi} \sum_{j=1}^{n} N\left(A_{j}\right) d \theta^{\prime}=\frac{1}{2 \pi} \cdot 2 \pi \sum_{j=1}^{n} N\left(A_{j}\right) \\
& =\sum_{j=1}^{n} N\left(A_{j}\right)
\end{aligned}
$$

Atente-se a proposição que exibiremos seguidamente à Fórmula de Gelfand apresentada abaixo.

Teorema 4.21 (Fórmula de Gelfand) Seja $A \in \operatorname{Mat}(n, \mathbb{C})$ e $\|\cdot\|$ uma norma matricial, então

$$
\rho(A)=\lim _{n \rightarrow \infty}\left\|A^{n}\right\|^{\frac{1}{n}}
$$

Proposição 4.22 Seja $A \in S L(2, \mathbb{R})$, então

$$
\log \rho(A)=\lim _{n \rightarrow \infty} \frac{1}{n} \log \left\|A^{n}\right\|=\lim _{n \rightarrow \infty} \frac{N\left(A^{n}\right)}{n} .
$$

Prova. Pela fórmula de Gelfand temos, para qualquer norma matricial, em particular para norma do operador, o seguinte resultado $\rho(A)=\lim _{n \rightarrow \infty}\left\|A^{n}\right\|^{\frac{1}{n}}$, e portanto segue que $\log \rho(A)=\lim _{n \rightarrow \infty} \frac{1}{n} \log \left\|A^{n}\right\|$. Por outro lado, já que $A^{n} \in \mathrm{SL}(2, \mathbb{R})$ temos $\left\|A^{n}\right\| \geq 1$, e ainda obtemos $0<\left\|A^{n}\right\|^{-1} \leq 1 \leq\left\|A^{n}\right\|$. 
Consequentemente

$$
\begin{aligned}
\log \left\|A^{n}\right\|+\log \frac{1}{2} & =\log \frac{\left\|A^{n}\right\|}{2} \leq \log \left(\frac{\left\|A^{n}\right\|}{2}+\frac{\left\|A^{n}\right\|^{-1}}{2}\right)=N\left(A^{n}\right) \\
& =\log \left(\frac{\left\|A^{n}\right\|+\left\|A^{n}\right\|^{-1}}{2}\right) \leq \log \left(\frac{\left\|A^{n}\right\|+\left\|A^{n}\right\|}{2}\right)=\log \left\|A^{n}\right\|,
\end{aligned}
$$

ou seja,

$$
\log \left\|A^{n}\right\|+\log \frac{1}{2} \leq N\left(A^{n}\right) \leq \log \left\|A^{n}\right\|
$$

Portanto,

$\lim _{n \rightarrow \infty} \frac{1}{n} \log \left\|A^{n}\right\|=\lim _{n \rightarrow \infty} \frac{1}{n}\left(\log \left\|A^{n}\right\|+\log \frac{1}{2}\right) \leq \lim _{n \rightarrow \infty} \frac{N\left(A^{n}\right)}{n} \leq \lim _{n \rightarrow \infty} \frac{1}{n} \log \left\|A^{n}\right\|$.

Observação 4.23 A relação interessante que o Corolário 4.20 apresenta com respeito ao Teorema 4.19, é o fato de que, munido à Proposição anterior, 4.22, o Teorema 4.19 pode também ser deduzido facilmente como um corolário do

Teorema 4.20.

Prova. Sejam $A_{1}, \cdots, A_{n} \in \mathrm{SL}(2, \mathbb{R})$ e defina as funções

$$
\begin{aligned}
B:[0,2 \pi] & \longrightarrow \mathrm{SL}(2, \mathbb{R}) \\
\theta & \longmapsto B(\theta)=B_{\theta}=\prod_{j=1}^{n} A_{j} R_{\theta} \\
f_{m}:[0,2 \pi] & \longrightarrow \mathbb{R} \\
\theta & \longmapsto \frac{1}{m} N\left(B_{\theta}^{m}\right)=\frac{1}{m} \log \left(\frac{\left\|B_{\theta}^{m}\right\|+\left\|B_{\theta}^{m}\right\|^{-1}}{2}\right)
\end{aligned}
$$

para todo $m \in \mathbb{N}$. Note, para $j \in\{1, \ldots, n\}$ as matrizes $A_{j}$ podem ser vistas como constantes em $\operatorname{SL}(2, \mathbb{R})$, portanto a função $\theta \mapsto A_{j} R_{\theta}$ é contínua para cada $j$, assim $B$ é uma função contínua e portanto $f_{m}$ tratase também de uma função contínua para todo $m \in \mathbb{N}$. Deste modo, para todo $m \in \mathbb{N}$, a função $f_{m}$ é mensurável com respeito a Lebesgue e como $B_{\theta} \in \mathrm{SL}(2, \mathbb{R})$ para todo $\theta \in[0,2 \pi]$, segue que $\left\|B_{\theta}\right\| \geq 1 \mathrm{e}$ $\left\|B_{\theta}\right\|^{-1} \leq 1 \leq\left\|B_{\theta}\right\|$, então

$$
\begin{aligned}
f_{m}(\theta) & =\frac{1}{m} N\left(B_{\theta}^{m}\right)=\frac{1}{m} \log \left(\frac{\left\|B_{\theta}^{m}\right\|+\left\|B_{\theta}^{m}\right\|^{-1}}{2}\right) \\
& \leq \frac{1}{m} \log \left(\left\|B_{\theta}^{m}\right\|\right) \leq \frac{1}{m} \log \left(\left\|B_{\theta}\right\|^{m}\right)=\log \left\|B_{\theta}\right\| .
\end{aligned}
$$


Além disso, note que para todo $m \in \mathbb{N},\left\|B_{\theta}^{m}\right\|>0$ e portanto para o conjunto $\left\{\left\|B_{\theta}^{m}\right\|,\left.\left\|B_{\theta}^{m}\right\|\right|^{-1}\right\}$, a média aritmética é maior ou igual que a média geométrica, ou seja,

$$
\frac{\left\|B_{\theta}^{m}\right\|+\left\|B_{\theta}^{m}\right\|^{-1}}{2} \geq \sqrt{\left\|B_{\theta}^{m}\right\|\left\|B_{\theta}^{m}\right\|^{-1}}=1,
$$

com isso $N\left(B_{\theta}^{m}\right) \geq 0$, consequentemente $f_{m}(\theta)=\left|f_{m}(\theta)\right|$, então para todo $\theta \in[0,2 \pi]$ e $m \in \mathbb{N}$ temos $\left|f_{m}(\theta)\right| \leq \log \left(\| B_{\theta}||\right)$, sendo $\log \left(\left\|B_{\theta}\right\|\right)$ ainda uma função integrável com respeito à Lebesgue, pois para todo $\theta \in[0,2 \pi]$ temos $\left\|B_{\theta}\right\| \leq \prod_{j=1}^{n}\left\|A_{j} R_{\theta}\right\|=\prod_{j=1}^{n}\left\|A_{j}\right\|<\infty$, portanto o Teorema da Convergência Dominada é aplicável, e este munido à Proposição 4.22 nos fornece

$$
\begin{aligned}
\frac{1}{2 \pi} \int_{0}^{2 \pi} \log \rho\left(B_{\theta}\right) d \theta & =\frac{1}{2 \pi} \int_{0}^{2 \pi} \lim _{m \rightarrow \infty} \frac{1}{m} N\left(B_{\theta}^{m}\right) d \theta \\
& =\frac{1}{2 \pi} \int_{0}^{2 \pi} \lim _{m \rightarrow \infty} \frac{1}{m} N\left(\left(A_{n} R_{\theta} \cdots A_{1} R_{\theta}\right)^{m}\right) d \theta \\
& =\lim _{m \rightarrow \infty} \int_{0}^{2 \pi} \frac{1}{m} N\left(\left(A_{n} R_{\theta} \cdots A_{1} R_{\theta}\right)^{m}\right) d \theta \\
& =\lim _{m \rightarrow \infty} \frac{1}{m} m\left(N\left(A_{n}\right)+\cdots N\left(A_{1}\right)\right) \\
& =\sum_{j=1}^{n} N\left(A_{j}\right)
\end{aligned}
$$

e então, o Teorema 4.19 está provado.

No decorrer deste texto e das demonstrações exibidas, já realizamos cálculos relacionados ao que veremos a seguir, no entanto achamos interessante evidenciar este por meio de um lema, já que é usado diretamente no Teorema subsequente.

Lema 4.24 Seja $A \in S L(2, \mathbb{R})$, então $N(A) \leq \log \|A\|<\log 2+N(A)$.

Prova. Como já provado, para $A \in \mathrm{SL}(2, \mathbb{R})$ temos $\|A\| \geq 1$ e portanto $\|A\|^{-1} \leq 1 \leq\|A\|$. Assim

$$
N(A)=\log \left(\frac{\|A\|+\|A\|^{-1}}{2}\right) \leq \log \frac{\|A\|+\|A\|}{2}=\log \|A\| .
$$

Por outro lado,

$$
\log 2+N(A)=\log \left(2 \frac{\|A\|+\|A\|^{-1}}{2}\right)=\log \left(\|A \mid+\| A \|^{-1}\right)>\log \|A\|
$$

Enfim chegamos à Fórmula de Avila-Bochi-Herman. Para este resultado devemos estar sob uma transformação ergódica $(X, \mu, T)$ e um cociclo linear 
$A: X \rightarrow \mathrm{SL}(2, \mathbb{R})$, o qual satisfaz a seguinte condição de integrabilidade

$$
\int_{X} \log \|A(x)\| d \mu(x)<\infty \text {. }
$$

Com isso, para $\theta \in \mathbb{R}$ definimos o seguinte cociclo linear

$$
\begin{aligned}
A R_{\theta}: X & \longrightarrow \mathrm{SL}(2, \mathbb{R}) \\
x & \longmapsto\left(A R_{\theta}\right)(x)=A(x) R_{\theta} .
\end{aligned}
$$

Note ainda que a função $[0,2 \pi] \ni \theta \mapsto L\left(A R_{\theta}\right)$ trata-se de uma função $\mu$-mensurável, já que é o limite de funções mensuráveis.

Teorema 4.25 (Fórmula de Avila-Bochi-Herman) Seja $T: X \rightarrow X$ uma transformação ergódica e $A: X \rightarrow S L(2, \mathbb{R})$ uma função mensurável, tal que $\log \|A\| \in L_{\mu}^{1}(X)$. Então,

$$
\frac{1}{2 \pi} \int_{0}^{2 \pi} L\left(A R_{\theta}\right) d \theta=\int_{X} \log \left(\frac{\|A(x)\|+\|A(x)\|^{-1}}{2}\right) d \mu(x)
$$

Prova. Pelo Corolário 4.20 e pelo Lema 4.24, segue para $x \in X$

$$
\begin{aligned}
\sum_{j=0}^{n-1} N\left(A\left(T^{j}(x)\right)\right) & =\frac{1}{2 \pi} \int_{0}^{2 \pi} N\left(A\left(T^{n-1}(x)\right) R_{\theta} \cdots A(x) R_{\theta}\right) d \theta \\
& =\frac{1}{2 \pi} \int_{0}^{2 \pi} N\left(\left(A R_{\theta}\right)^{(n)}(x)\right) d \theta \\
& \leq \frac{1}{2 \pi} \int_{0}^{2 \pi} \log \left\|\left(A R_{\theta}\right)^{(n)}(x)\right\| d \theta
\end{aligned}
$$

e ainda

$$
\begin{aligned}
\sum_{j=0}^{n-1} N\left(A\left(T^{j}(x)\right)\right) & =\frac{1}{2 \pi} \int_{0}^{2 \pi} N\left(A\left(T^{n-1}(x)\right) R_{\theta} \cdots A(x) R_{\theta}\right) d \theta \\
& =\frac{1}{2 \pi} \int_{0}^{2 \pi} N\left(\left(A R_{\theta}\right)^{(n)}(x)\right) d \theta \\
& =\frac{1}{2 \pi} \int_{0}^{2 \pi} \log \left(\frac{\left\|\left(A R_{\theta}\right)^{(n)}(x)\right\|+\left\|\left(A R_{\theta}\right)^{(n)}(x)\right\|^{-1}}{2}\right) d \theta \\
& =\frac{1}{2 \pi} \int_{0}^{2 \pi} \log \left(\left\|\left(A R_{\theta}\right)^{(n)}(x)\right\|+\left\|\left(A R_{\theta}\right)^{(n)}(x)\right\|^{-1}\right)-\log 2 d \theta \\
& =\frac{1}{2 \pi} \int_{0}^{2 \pi} \log \left(\left\|\left(A R_{\theta}\right)^{(n)}(x)\right\|+\left\|\left(A R_{\theta}\right)^{(n)}(x)\right\|^{-1}\right) d \theta-\log 2 \\
& \geq \frac{1}{2 \pi} \int_{0}^{2 \pi} \log \left\|\left(A R_{\theta}\right)^{(n)}(x)\right\| d \theta-\log 2
\end{aligned}
$$


a última desigualdade segue do fato de que a função logarítimica é crescente. Consequentemente, temos

$\sum_{j=0}^{n-1} N\left(A\left(T^{j}(x)\right)\right) \leq \frac{1}{2 \pi} \int_{0}^{2 \pi} \log \left\|\left(A R_{\theta}\right)^{(n)}(x)\right\| d \theta \leq \log 2+\sum_{j=0}^{n-1} N\left(A\left(T^{j}(x)\right)\right)$

Então

$$
\begin{aligned}
\lim _{n \rightarrow \infty} \frac{1}{n} \sum_{j=0}^{n-1} N\left(A\left(T^{j}(x)\right)\right) & \leq \lim _{n \rightarrow \infty} \frac{1}{n} \frac{1}{2 \pi} \int_{0}^{2 \pi} \log \left(\left\|\left(A R_{\theta}\right)^{(n)}(x)\right\|\right) d \theta \\
& \leq \lim _{n \rightarrow \infty} \frac{1}{n} \log 2+\lim _{n \rightarrow \infty} \frac{1}{n} \sum_{j=0}^{n-1} N\left(A\left(T^{j}(x)\right)\right) \\
& =\lim _{n \rightarrow \infty} \frac{1}{n} \sum_{j=0}^{n-1} N\left(A\left(T^{j}(x)\right)\right)
\end{aligned}
$$

isto é,

$$
\lim _{n \rightarrow \infty} \frac{1}{2 \pi} \int_{0}^{2 \pi} \frac{1}{n} \log \left\|\left(A R_{\theta}\right)^{(n)}(x)\right\| d \theta=\lim _{n \rightarrow \infty} \frac{1}{n} \sum_{j=0}^{n-1} N\left(A\left(T^{j}(x)\right)\right)
$$

Note que a função $N \circ A: X \rightarrow \mathbb{R}$, definida por $N(A)=\log \left(\frac{\|A\|+\|A\|^{-1}}{2}\right)$, é $\mu$-integrável, $N(A) \in L^{1}(\mu, X)$, já que $N(A) \leq \log \|A\|$, o que implica no fato de $\int_{X} N(A) d \mu \leq \int_{X} \log \|A\| d \mu<\infty$. Além disso, $T$ é uma transformação ergódica definida sobre o espaço de probabilidade $(X, \mu)$. Assim, faz sentido aplicarmos o Teorema de Birkhoff, Teorema 3.9, portanto

$$
\lim _{n \rightarrow \infty} \frac{1}{n} \sum_{j=0}^{n-1} N\left(A\left(T^{j}(x)\right)\right)=\int_{X} N(A(x)) d \mu(x) \quad \mu-\text { q.t.p. } x \in X
$$

Deste modo,

$$
\lim _{n \rightarrow \infty} \frac{1}{2 \pi} \int_{0}^{2 \pi} \frac{1}{n} \log \left\|\left(A R_{\theta}\right)^{(n)}(x)\right\| d \theta=\int_{X} N(A(x)) d \mu(x) \quad \mu \text { - q.t.p. } x \in X
$$

Perceba, para finalizarmos a prova deste teorema, basta aplicarmos o teorema da convergência dominada, mas antes precisamos provar que, de fato, faz sentido aplicarmos este teorema. Temos para todo $x \in X$ e para todo $\theta \in[0,2 \pi]$

$$
\begin{aligned}
0 \leq \frac{1}{n} \log \left\|\left(A R_{\theta}\right)^{(n)}(x)\right\| & =\frac{1}{n} \log \left(\left\|A\left(T^{n-1}(x)\right) R_{\theta} \cdots A(x) R_{\theta}\right\|\right) \\
& \leq \frac{1}{n} \log \left(\left\|A\left(T^{n-1}(x)\right)\right\|\left\|R_{\theta}\right\| \cdots\|A(x)\|\left\|R_{\theta}\right\|\right) \\
& =\frac{1}{n} \log \left(\left\|A\left(T^{n-1}(x)\right)\right\| \cdots\|A(x)\|\right)
\end{aligned}
$$




$$
=\frac{1}{n} \sum_{j=0}^{n-1} \log \left\|A\left(T^{j}(x)\right)\right\|
$$

Defina as funções

$$
\begin{aligned}
h_{n}: X & \longrightarrow \mathbb{R} \\
x & \mapsto \frac{1}{n} \log \left\|\left(A R_{\theta}\right)^{(n)}(x)\right\|,
\end{aligned}
$$

e

$$
\begin{aligned}
f_{n}: X & \longrightarrow \mathbb{R} \\
x & \mapsto \frac{1}{n} \sum_{j=0}^{n-1} \log \left\|A\left(T^{j}(x)\right)\right\|
\end{aligned}
$$

Note que, por hipótese, $h_{n}$ é uma função $\mu$-mensurável, em particular é $\mu$-integrável, para todo $n \in \mathbb{N}$. Portanto basta encontrarmos uma função $g \in L^{1}(\mu, X)$ tal que $\left|h_{n}(x)\right|<|g(x)|$, para todo $n \in \mathbb{N}$ e $\mu$-q.t.p. $x \in X$.

Pelo Teorema de Birkhoff,

$\lim _{n \rightarrow \infty} f_{n}(x)=\lim _{n \rightarrow \infty} \frac{1}{n} \sum_{j=0}^{n-1} \log \left\|A\left(T^{j}(x)\right)\right\|=\int_{X} \log \|A(x)\| d \mu \mu-$ q.t.p. $x \in X$

Então $\left(f_{n}\right)_{n \in \mathbb{N}}$ é uma sequência convergente, consequentemente limitada. Deste modo, existe uma constante $c \in \mathbb{R}$ tal que $\left|f_{n}(x)\right| \leq \int_{X} \log \|A(x)\| d \mu+c$, para todo $n \in \mathbb{N}$ e $\mu$-q.t.p. $x \in X$. Assim, defina

$$
\begin{aligned}
g: X & \longrightarrow \mathbb{R}^{*} \\
x & \mapsto \int_{X} \log \|A(x)\| d \mu+c
\end{aligned}
$$

uma função claramente integrável.

Consequentemente $\left|h_{n}(x)\right| \leq\left|f_{n}(x)\right| \leq g(x) \leq|g(x)|$, para todo $n \in \mathbb{N}$ e $\mu$-q.t.p. $x \in X$. Portanto, pelo Teorema da Convergência Dominada, temos

$$
\begin{aligned}
& \frac{1}{2 \pi} \int_{0}^{2 \pi} L\left(A R_{\theta}\right) d \theta=\lim _{n \rightarrow \infty} \frac{1}{2 \pi} \int_{0}^{2 \pi} \frac{1}{n} \log \left\|\left(A R_{\theta}\right)^{(n)}(x)\right\| d \theta=\int_{X} N(A(x)) d \mu(x), \\
& \mu-\text { q.t.p. } x \in X .
\end{aligned}
$$

Apesar de tratar-se de um teorema relevante, a princípio podemos nos questionar sobre o real efeito do mesmo, ou o que de fato o teorema pode nos informar à respeito do expoente de Lyapunov maximal do cociclo $A: X \rightarrow \mathrm{SL}(2, \mathbb{R})$, ao invés do cociclo $A R_{\theta}: X \rightarrow \mathrm{SL}(2, \mathbb{R})$ dado por 
$\left(A R_{\theta}\right)(x)=A(x) R_{\theta}$, como vimos. Deste modo, é pertinente exibirmos uma aplicação simples da Fórmula de Avila-Bochi-Herman, a qual nos permite explicitar o Expoente de Lyapunov maximal de um cociclo já conhecido.

Corolário 4.26 Considere o cociclo linear apresentado na primeira desigualdade de Herman, Teorema 2.2, em que $T: S^{1} \rightarrow S^{1}$ é uma rotação irracional por um ângulo $\omega$ e $A: S^{1} \rightarrow S L(2, \mathbb{R})$ é o cociclo linear dado por $A\left(e^{i \theta}\right)=A_{0} R_{\theta}$ com $A_{0}=\left(\begin{array}{cc}\delta & 0 \\ 0 & \delta^{-1}\end{array}\right)$ para algum $\delta>0$. Então

$$
L(A)=\log \left(\frac{\delta+\delta^{-1}}{2}\right)
$$

Prova. Para $z=e^{i t} \in S^{1}$ e $\theta \in[0,2 \pi]$ temos

$$
\begin{aligned}
\left(A R_{\theta}\right)^{(n)}(z) & =\left(A R_{\theta}\right)\left(T^{n-1}(z)\right) \ldots\left(A R_{\theta}\right)(T(z))\left(A R_{\theta}\right)(z) \\
& =A\left(T^{n-1}(z)\right) R_{\theta} \ldots A(T(z)) R_{\theta} A(z) R_{\theta} \\
& =A\left(e^{i(t+(n-1) \omega)}\right) R_{\theta} \ldots A\left(e^{i(t+\omega)}\right) R_{\theta} A\left(e^{i(t)}\right) R_{\theta} \\
& =A_{0} R_{\theta+t+(n-1) \omega} \ldots A_{0} R_{\theta+t+\omega} A_{0} R_{\theta+t} \\
& =A\left(e^{i(\theta+t+(n-1) \omega)}\right) A\left(e^{i(\theta+t+\omega)}\right) A\left(e^{i(\theta+t)}\right) \\
& =A\left(T^{n-1}\left(e^{i \theta} z\right)\right) A\left(T\left(e^{i \theta} z\right)\right) A\left(e^{i \theta} z\right) \\
& =A^{(n)}\left(e^{i \theta} z\right) .
\end{aligned}
$$

Portanto,

$$
L\left(A R_{\theta}\right)=\lim _{n \rightarrow \infty} \frac{1}{n} \log \left\|A R_{\theta}^{(n)}(z)\right\|=\lim _{n \rightarrow \infty} \frac{1}{n} \log \left\|A^{(n)}\left(e^{i \theta} z\right)\right\|=L(A),
$$

$\mu$-q.t.p., já que $T$ é uma transformação ergódica.

Portanto, $L\left(A R_{\theta}\right)=L(A) \mu$-q.t.p. e para todo $\theta \in[0,2 \pi]$, assim pela Fórmula de Avila-Bochi-Herman temos

$$
\begin{aligned}
L(A)=L\left(A R_{\theta}\right) & =\frac{1}{2 \pi} \int_{0}^{2 \pi} L\left(A R_{\theta}\right) d \theta=\int_{S^{1}} \log \left(\frac{\|A(x)\|+\|A(x)\|^{-1}}{2}\right) d \mu(x) \\
& =\int_{S^{1}} \log \left(\frac{\left\|A_{0}\right\|+\left\|A_{0}\right\|^{-1}}{2}\right) d \mu=\log \left(\frac{\delta+\delta^{-1}}{2}\right) .
\end{aligned}
$$




\section{Referências bibliográficas}

[1] AVILA, A.. Density of positive Lyapunov exponents for $\operatorname{SL}(2, \mathbb{R})$ cocycles. Journal of the American Mathematical Society, 24(4):16, 2011. 1

[2] AVILA, A.; BOCHI, J.. A formula with some applications to the theory of Lyapunov exponents. Israel Journal of Mathematics, 131:13, 2002. 1

[3] ADAMS, C.; FRAnZOSA, R.. Introduction to Topology: Pure and Applied. Pearson, 2009. 3.4

[4] BARAVIERA, A.; DiAS, J.; DUARTE, P.. On the Herman-AvilaBochi formula for Lyapunov exponents of $\mathrm{SL}(2, \mathbb{R})$-cocycles. Nonlinearity, 24(9):13, 2011. 1

[5] CONRAD, K.. Decomposing $\mathbf{S L}(2, \mathbb{R})$. https://kconrad.math . uconn.edu/blurbs/grouptheory/SL $(2, R)$.pdf. 3.4

[6] CONWAY, J. B.. Functions of One Complex Variable. Graduate Texts in Mathematics. Springer-Verlag, 1973. 3.3

[7] CONWAY, J. B.. Functions of One Complex Variable II. Graduate Texts in Mathematics. Springer-Verlag, 1995. 3.3

[8] DUARTE, P.; KLEIN, S.. Continuity of the Lyapunov Exponents of Linear Cocycles. Publicações Matemáticas, 31 ○ Colóquio Brasileiro de Matemática. IMPA, 2017. 3.2

[9] GAMA, L.. Quasi-periodicity and the positivity of Lyapunov exponents. Master's thesis, Pontifícia Universidade Católica do Rio de Janeiro, 2018. 3.3

[10] HALL, B.. Lie Groups, Lie Algebras, and Representations: An Elementary Introduction, 2nd edn. Graduate Texts in Mathematics. Springer, 2015. 3.4

[11] HATCHER, A.. Algebraic Topology. Cambridge University Press, 2002. 3.4

[12] HELGASON, S.. Differential Geometry, Lie Groups, and Symmetric Spaces. Academic Press, 1978. 3.4 
[13] MUNKERS, J.. Topology. Modern Classics for Advanced Mathematics Series. Pearson, 2000. 3.4, 3.4, 3.4

[14] SADEL, C.. A Herman-Avila-Bochi formula for higherdimensional pseudo-unitary and hermitian-symplectic cocycles. Ergodic Theory and Dynamical Systems, 35(5):10, 2013. 1

[15] VIANA, M.. Lectures on Lyapunov Exponents. Cambridge studies in advanced mathematics. Cambridge University Press, 2014. 3.2

[16] VIANA, M.; OLIVEIRA, K.. Fundamentos da Teoria Ergódica. Fronteiras da Matemática. SBM, 2019. 3.1

[17] WALTERS, P.. An Introduction to Ergodic Theory. Graduate Texts in Mathematics. Springer, 2000. 3.1

[18] ZHANG, Z.. Positive Lyapunov exponents for quasi-periodic szegő cocycles. Nonlinearity, 25(6):28, 2012. 1 